\title{
Quantum field theory on projective modules
}

\author{
Victor Gayral, Jan-Hendrik Jureit, Thomas Krajewski, and Raimar Wulkenhaar
}

\begin{abstract}
We propose a general formulation of perturbative quantum field theory on (finitely generated) projective modules over noncommutative algebras. This is the analogue of scalar field theories with non-trivial topology in the noncommutative realm. We treat in detail the case of Heisenberg modules over noncommutative tori and show how these models can be understood as large rectangular $p \times q$ matrix models, in the limit $p / q \rightarrow \theta$, where $\theta$ is a possibly irrational number. We find out that the model is highly sensitive to the number theoretical aspect of $\theta$ and suffers from an UV/IR-mixing. We give a way to cure the entanglement and prove 1-loop renormalisability.
\end{abstract}

Mathematics Subject Classification (2000). 46L87, 46L85, 81T75, 81T15.

Keywords. Heisenberg modules, renormalisation, matrix models, Diophantine condition.

\section{Contents}

1 Introduction . . . . . . . . . . . . . . . . . . . . 431

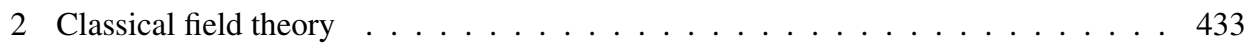

3 QFT and diagrammatics . . . . . . . . . . . . . . . . . . . . . . . . . . . . . . . . . . . . . . . . . . . . . . . .

4 One-loop renormalisation . . . . . . . . . . . . . . . . . . . 471

5 Mixed diagrams . . . . . . . . . . . . . . . . . . . . . . 488

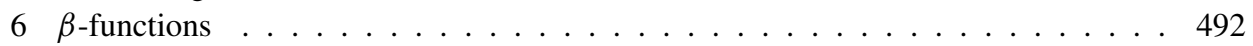

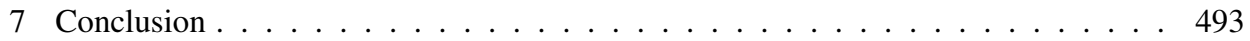

References . . . . . . . . . . . . . . . . . . . . . 494

\section{Introduction}

In its most general acceptance, quantum field theory (QFT) can be defined as the theory of infinite-dimensional dynamical systems which are based on geometrical concepts like, for instance, locality and invariance principles. Within the path integral approach, one first defines an action functional $S[\phi]$, the argument of which is a field $\phi$ that belongs to a fixed configuration space $\mathcal{C}$. Then one defines and studies 
the (euclidian) path integral

$$
\langle\mathcal{F}\rangle=\frac{\int_{e}[\mathscr{D} \phi] \mathcal{F}[\phi] e^{-S[\phi]}}{\int_{\mathscr{C}}[\mathcal{D} \phi] e^{-S[\phi]}},
$$

where $\mathcal{F}$ belongs to a suitable class of functionals of the field. Such a framework is at the root of our current understanding of the dynamics of elementary particles, where the configuration space contains matter fields (section of various bundles over space-time) and gauge fields (connections over these bundles). For a trivial topology of these bundles, all these fields can be understood as functions over the space-time manifold $\mathcal{M}$. Then the action functional involves the integral over $\mathcal{M}$ of a Lagrangian density ultimately constructed out of pointwise products of the fields.

Over the last decade a radical modification of this construction has proven to be of interest in mathematical physics: instead of being grounded in ordinary differential geometry, QFT can also fruitfully use the concepts introduced in Noncommutative Geometry. The latter is a branch of mathematics pioneered in the eighties by A. Connes (see [10] and [12] for an overview of recent developments) which extends geometrical concepts to a wide class of spaces whose coordinate algebras are noncommutative instead of being merely functions with the pointwise product. For instance, noncommutative analogues of vector bundles, which are the natural receptacles for matter fields, are defined as projective modules over the noncommutative algebra of coordinates. Noncommutative field theory (NCFT) has grown up from the need of new methods in understanding a wide range of problems in theoretical physics, ranging from the construction of open string field theory [28] to the understanding of the dynamics of string theory [27] and M-theory [11] in magnetic backgrounds. Though not directly related to NCFT, other applications of noncommutative geometry include the geometry of aperiodic solids [3] as well as the standard model of particle physics [5].

The NCFT's involved in most of these applications are based on configuration spaces $\mathcal{C}$ which consist of matrices with coefficients in the algebra of quantum coordinates $\mathcal{A}$. The latter are deformations of the commutative algebra of coordinates over space-time, with deformation parameters depending on some background. In the language of noncommutative geometry, these configuration spaces correspond to free modules, which are the analogues of trivial vector bundles. There is first success with renormalisation to all orders of such NCFT's [18]. Since noncommutative geometry is versatile enough to include non-trivial bundles as projective modules, the restriction to free modules appears to be rather unnatural. While projective modules already appear in the string theory literature (see for instance [29] and [27]), the NCFT's they naturally define have not yet been investigated. The present work aims at filling this gap in the case of the simplest non-trivial projective modules over noncommutative tori. 
This paper is organised as follows.

In Section 2 we first give a general construction of the configuration space and the action functional in the framework of noncommutative geometry, making use of a spectral triple $(\mathcal{A}, \mathscr{H}, \mathscr{D})$. Then we illustrate the general theory for some Heisenberg modules over $d$-dimensional noncommutative tori. These modules are constructed using representations of the Heisenberg group that can be formulated either using functions over $\mathbb{R}^{\frac{d}{2}}$ or holomorphic functions on $\mathbb{C}^{\frac{d}{2}}$. In this last case we illustrate how such a module appears naturally in the study of an electron on a plane in an external magnetic field which is confined to its ground state in presence of a periodic potential.

Section 3 is devoted to the construction of the NCFT based on Heisenberg modules. We first give the perturbative expansion of the path integral using a HubbardStratonovitch transformation. The resulting theory makes use of planar diagrams reminiscent of rectangular matrix models and exhibits a duality symmetry. Then we use the position space formulation to strengthen this analogy with rectangular matrices. Finally, we give the general rules for the computation of Feynman diagrams in the holomorphic representation.

In Section 4 we use the holomorphic representation to compute 1-loop diagrams. While the divergent planar diagrams turn out to be renormalisable by standard methods, new phenomena occur in the non-planar case. If the deformation parameter fulfils a Diophantine condition, we give explicit bounds on the amplitude showing that they are renormalised by an extra counterterm.

Section 5 deals with the effect of the new counterterm. It is shown that it is harmless and does not spoil the general properties of the NCFT.

Finally, in Section 6 we compute the $\beta$-functions for the two interacting terms.

\section{Classical field theory}

2.1. Projective modules in noncommutative geometry. To begin with, let us recall some basic facts about projective modules in noncommutative geometry and the way they enter in the construction of a noncommutative field theory. A much more thorough presentation of the subject can be found in [10] (see also [12] for an overview of recent developments in noncommutative geometry). Here we restrict ourselves to the amount of material that is necessary in order to construct our noncommutative field theory.

First we recall that the basic idea of noncommutative geometry is to replace the commutative data of a space $\mathcal{X}$ by a possibly noncommutative algebra $\mathcal{A}$ that plays the rôle of the complex-valued functions on $\mathcal{X}$. In general, it is necessary to assume that $\mathcal{A}$ is a $\mathrm{C}^{*}$-algebra, which means that it is equipped with an involution $*$ and a norm which are compatible. This is motivated by the Gelfand-Naimark theorem which 
asserts that commutative $\mathrm{C}^{*}$-algebras with unit are equivalent to compact Hausdorff spaces.

In the same spirit the notion of a finite-dimensional complex vector bundle is extended to the noncommutative realm by first looking at the structure of its space of sections. By the Serre-Swan theorem, spaces of sections of vector bundles over $\mathcal{X}$ are equivalent to finitely generated projective modules $\mathcal{E}$ (projective modules for short) over the algebra of continuous functions over $\mathcal{X}$. Up to an isomorphism such a module can always be realised as the right $\mathscr{A}$-module $e \mathcal{A}^{N}$, where $e$ is a projection in the algebra of $N \times N$ matrices with coefficients in $\mathcal{A}$. The relation between the idempotent and the standard construction of a vector bundle using transition functions goes as follows. Cover the compact space $\mathcal{X}$ by $N$ open sets $\left\{U_{i}\right\}$ defining a good cover and let $\sum_{i}\left|f_{i}\right|^{2}$ be a partition of unity associated to this cover. Using the transition functions $g_{i j}$, we define the $N \times N$ matrix $e_{i j}=f_{i}^{*} g_{i j} f_{j}$, whose entries are complexvalued functions over $\mathcal{X}$. Then it is straightforward to check that $e$ is a projection using the cocycle condition $g_{i j}=g_{i k} g_{k j}$. Accordingly, in the general setting one defines by duality a noncommutative vector bundle to be a finitely generated projective module over a possibly noncommutative $C^{*}$-algebra. From a classical field theoretical perspective, the module $\mathcal{E}$ is the natural receptacle for the matter fields with non-trivial topology. If the topology is trivial, these fields simply live in a free module $\mathcal{A}^{N}$, the simplest version being the algebra itself.

Rather than at the $\mathrm{C}^{*}$-level we will work here at the smooth one, i.e., we will assume that $\mathcal{A}$ is a Fréchet pre-C*-algebra. This means that $\mathcal{A}$ is a dense sub-algebra of a $C^{*}$-algebra $A$, which is stable under holomorphic functional calculus and which is endowed with a Fréchet topology. We will assume that the topology comes from a set of semi-norms, say $\left\{p_{i}\right\}$. Without any further structure, $\mathcal{E}$ is simply a projective right module over $\mathcal{A}$; it will be promoted to a topological vector space later on. There are many reasons why it is preferable to work at the smooth level. First, our construction of perturbative field theory relies on the theory of distributions, which is useless at the level of $\mathrm{C}^{*}$-algebras and $\mathrm{C}^{*}$-modules (our projective modules will soon be endowed with an $\mathcal{A}$-valued hermitian pairing promoting it to a pre-C*-module). But also for many interesting examples (e.g. Heisenberg modules and $\mathrm{C}^{*}$-dynamical systems [8]) one does not lose any geometrical information by passing from $\mathrm{C}^{*}$ - to smooth structures.

To construct a noncommutative field theory out of the previous data, one further needs a suitable space of differential forms as well as some scalar products on these forms. Differential forms are defined through a graded differential algebra

$$
\Omega(\mathcal{A}):=\bigoplus_{n \in \mathbb{N}} \Omega_{n}(\mathcal{A})
$$

which is a graded bi-module over $\mathcal{A}$, together with a nilpotent differential operator

$$
\mathrm{d}: \Omega_{n}(\mathcal{A}) \rightarrow \Omega_{n+1}(\mathcal{A}),
$$


fulfilling the graded Leibniz rule. Note that in the general noncommutative setting, it is not assumed that $\Omega(\mathcal{A})$ is graded commutative. Usually one assumes that $\Omega_{0}(\mathcal{A})=\mathcal{A}$ and that $\Omega(\mathcal{A})$ is equipped with an involution $*$ compatible with those of $\mathcal{A}$. The scalar product $\langle\cdot, \cdot\rangle_{n}$ on $\Omega_{n}(\mathcal{A})$ is assumed to be compatible with the left and right actions of $\mathcal{A}$,

$$
\begin{aligned}
& \langle\omega, \eta a\rangle_{n}=\left\langle\omega a^{*}, \eta\right\rangle_{n}, \\
& \langle\omega, a \eta\rangle_{n}=\left\langle a^{*} \omega, \eta\right\rangle_{n},
\end{aligned}
$$

for all $\omega, \eta \in \Omega_{n}(\mathcal{A})$ and $a \in \mathcal{A}$. In full generality, the scalar product in degree 0 is always obtained from a faithful state $\Psi$, i.e., a normalised positive-definite linear functional on $\mathcal{A}$ :

$$
\langle a, b\rangle_{0}:=\Psi\left(a^{*} b\right), \quad a, b \in \mathcal{A} .
$$

In view of the application we have in mind, it is more appropriate to require that the state is actually a faithful trace on $\mathcal{A}$ so that

$$
\langle a, b\rangle_{0}=\operatorname{Tr}_{\mathcal{A}}\left(a^{*} b\right) .
$$

For instance, in the simplest version of a (commutative or not) Yang-Mills theory one starts with a topologically trivial connection given by an anti-hermitian element $\mathrm{A}$ of $\Omega_{1}(\mathcal{A})$, out of which we define the curvature as $\mathrm{F}=\mathrm{dA}+\mathrm{A}^{2} \in \Omega_{2}(\mathcal{A})$. The Yang-Mills action is then constructed as $\langle\mathrm{F}, \mathrm{F}\rangle_{2}$, where $\langle\cdot, \cdot\rangle_{2}$ is an invariant scalar product on $\Omega_{2}(\mathcal{A})$. This invariance condition simply states that the left and right actions of $\mathcal{A}$ are compatible with the scalar product. This ensures gauge invariance, where gauge transformations are given by unitary elements $u \in \mathcal{A}$, acting as

$$
\begin{aligned}
& \mathrm{A} \rightarrow u^{-1} \mathrm{~A} u+u^{-1} \mathrm{~d} u, \\
& \mathrm{~F} \rightarrow u^{-1} \mathrm{~F} u .
\end{aligned}
$$

Such a scalar product encodes an information that goes beyond the topological level contained in the algebra $\mathcal{A}$ alone. For instance, in a four-dimensional Yang-Mills theory, it amounts to the choice of a conformal structure. Before we come to grips with such an issue, let us note that one can also define in full generality a noncommutative Chern-Simons theory out of a cyclic 3-cocycle [4] which is a convenient setting to develop open string field theory [28]. As a topological theory, the construction of Chern-Simons theory does not rely on such a scalar product.

Differential forms and their scalar products are conveniently constructed out of a spectral triple $(\mathcal{A}, \mathscr{D}, \mathscr{H})$. This involves a Hilbert space $\mathscr{H}$ carrying a representation $\pi$ of the algebra $\mathcal{A}$ by bounded operators and a self-adjoint unbounded operator $\mathscr{D}$ with compact resolvent. This data is constrained by compatibility conditions, allowing to reconstruct a smooth Riemannian manifold when $\mathcal{A}$ is commutative. For instance, the commutators of $\mathscr{D}$ with the elements of $\mathcal{A}$ must extend to bounded operators. For 
a spectral triple, there is a notion of dimension given by the growth of the eigenvalues of $|\mathscr{D}|$. More precisely, such a triple has spectral dimension $d$ if the resolvent of $\mathscr{D}$ belongs to the $d$-th weak Schatten ideal $\mathscr{L}^{d, \infty}(\mathscr{H})$. The latter is the ideal of compact operators whose sequence of singular values (in decreasing order and counted with multiplicity) are $\mathcal{O}\left(n^{-1 / d}\right)$. With a spectral triple, one defines differential forms as a representation of the universal differential algebra

$$
a_{0} \mathrm{~d} a_{1} \ldots \mathrm{d} a_{n} \mapsto \pi\left(a_{0}\right)\left[\mathscr{D}, \pi\left(a_{1}\right)\right] \ldots\left[\mathscr{D}, \pi\left(a_{n}\right)\right] .
$$

Such representation can be quite pathological since it may happen that the image of an element of the universal differential algebra is zero, whereas the image of the differential of this element is not! To overcome this problem, one has to divide the resulting algebra by a graded differential ideal (the so-called junk ideal). Fortunately we can ignore this point here. This is because the first non-trivial component of this ideal occurs at the level of two-forms only. However, this is highly relevant for a proper formulation of noncommutative Yang-Mills theory in this framework.

For a triple of spectral dimension $d$, there is a canonical way to define the scalar product between two differential forms in $\Omega_{n}(\mathcal{A})$ :

$$
\langle\omega, \eta\rangle_{n}=\operatorname{Tr}_{\text {Dix }}\left(\omega^{*} \eta(1+|\mathscr{D}|)^{-d}\right) .
$$

Here $\operatorname{Tr}_{\text {Dix }}$ is any of the Dixmier traces. Such an object is a singular trace defined on the ideal $\mathscr{L}^{1, \infty}(\mathscr{H})$ and is heuristically given by the coefficient of the logarithmic divergence of the ordinary operator trace (see [10] for more details on Dixmier traces). There is no point to enter here in the mathematical subtleties of the theory of Dixmier traces. This is because the operator $(1+|D|)^{-d}$ we will consider in our example belongs to the class of 'measurable operators'. The latter consists of elements of $\mathscr{L}^{1, \infty}(\mathscr{H})$ for which any Dixmier trace gives the same result.

It is important to know that the Dirac operator $\mathscr{D}$ encodes the metric aspect of noncommutative geometry and allows also to define the fermionic action for spinors $\psi \in \mathscr{H}$ as $\langle\psi, \mathscr{D} \psi\rangle_{\mathscr{H}}$.

It is worthwhile to mention that the use of a spectral triple to construct noncommutative field theory is highly convenient (mainly because it allows to define differential forms with scalar products in a canonical way) but not necessary. Indeed, our construction is much more general and works for any differential calculus with scalar products on each component.

Turning back to the projective module $\mathcal{E}$, one has to define a connection $\nabla$. This is an operator that extends to elements of $\varepsilon$ the differential of a given differential calculus $(\Omega(\mathcal{A}), \mathrm{d})$. In general, a connection is a linear map

$$
\nabla: \mathcal{E} \rightarrow \mathcal{E} \otimes_{\mathcal{A}} \Omega_{1}(\mathcal{A}),
$$

fulfilling the Leibniz rule

$$
\nabla(\phi a)=\nabla(\phi) a+\phi \otimes \mathrm{d} a,
$$


for any $\phi \in \mathcal{E}$ and $a \in \mathcal{A}$. The projective module $\mathcal{E}$ is further equipped with a hermitian structure $(\cdot, \cdot)_{\mathcal{A}}: \mathcal{E} \times \mathcal{E} \rightarrow \mathcal{A}$, which is an $\mathcal{A}$-valued sesquilinear form on $\mathcal{E}$, satisfying the following compatibility and positivity conditions:

$$
\begin{aligned}
(\phi, \chi a)_{\mathcal{A}} & =(\phi, \chi)_{\mathcal{A}} a, \\
(\phi, \chi)_{\mathcal{A}} & =(\chi, \phi)_{\mathcal{A}}^{*}, \\
(\phi, \phi)_{\mathcal{A}} & \geq 0,
\end{aligned}
$$

for all $\phi, \chi \in \mathcal{E}$ and $a \in \mathcal{A}$. We recall that $a$ is a positive element of $\mathcal{A}$ if it can be written as $a=b^{*} b$ with $b \in \mathcal{A}$. Besides, the connection has to be compatible with the hermitian structure in the sense that

$$
\mathrm{d}(\phi, \chi)_{\mathcal{A}}=(\nabla \phi, \chi)_{\mathcal{A}}+(\phi, \nabla \chi)_{\mathcal{A}}
$$

for all $\phi, \chi \in \mathcal{E}$. We would like to stress that the field theory we are constructing is Euclidean, precisely because the pairing (2.11) is positive definite.

As a side remark, let us note that one can define the curvature of $\nabla$ as $\mathrm{F}=\nabla^{2}$, where $\nabla$ has been extended by the Leibniz rule to $\mathcal{E}_{\otimes_{\mathcal{A}}} \Omega(\mathcal{A})$. This is the starting point for the development of a general gauge theory in the framework of noncommutative geometry.

Within the supplementary structure of an hermitian $\mathcal{A}$-valued pairing, $\mathcal{E}$ can be promoted to a topological vector space in different ways. When $\mathcal{A}$ comes from a $\mathrm{C}^{*}$ algebra $A, \mathcal{E}$ is called a pre-Hilbert module and it can be completed with respect to the norm $\|\phi\|:=\sqrt{\left\|(\phi, \phi)_{\mathcal{A}}\right\|}$, where $\|\cdot\|$ denotes the $\mathrm{C}^{*}$-norm of $A$. The resulting Banach space is obviously called a right Hilbert- or $\mathrm{C}^{*}$-module. One can also define a Fréchet topology on $\mathcal{E}$, finer than the $C^{*}$ one, via the semi-norms $\left\{p_{i}\right\}$ of $\mathcal{A}$ by setting $q_{i}(\phi):=\sqrt{p_{i}\left((\phi, \phi)_{\mathcal{A}}\right)}$ for all $\phi \in \mathcal{E}$. When $\mathcal{E}$ is complete with respect to this topology, which we will assume from now on, we call it a Fréchet projective right $\mathcal{A}$-module. Finally, when $\mathcal{A}$ possesses a faithful trace $\operatorname{Tr}_{\mathcal{A}}, \mathcal{E}$ can be completed to a Hilbert space $\mathscr{H}_{\mathcal{E}}$ using the scalar product $\langle\cdot, \cdot\rangle_{\mathcal{E}}$ obtained by composition of the trace with the hermitian structure

$$
\langle\phi, \chi\rangle_{\mathcal{E}}:=\operatorname{Tr}_{\mathcal{A}}\left[(\phi, \chi)_{\mathcal{A}}\right] .
$$

We use the notation $\mathscr{H}_{\mathcal{E}}$ for this Hilbert space in order to emphasise its canonical nature once a trace and an hermitian structure are given.

If we realise the projective module as $e \mathcal{A}^{N}$ for some hermitian idempotent $e \in M_{N}(\mathcal{A})$, then a connection is easily defined as $\nabla(e \xi):=e \mathrm{~d}(e \xi)+e \mathrm{~A} e \xi$, $\xi \in \mathcal{A}^{N}$, where $\mathrm{A}$ is an anti-hermitian matrix in $M_{N}(\mathcal{A})$. In this case, the hermitian structure is given by $(e \xi, e \zeta)_{\mathcal{A}}:=\xi^{*} e \zeta$, and the compatibility of the connection follows from $(e \mathrm{~A} e)^{*}=-e \mathrm{~A} e$.

Finally, let us construct an action functional for a classical field $\phi$ in a projective module $\mathcal{E}$. To this aim, we have to extend the scalar product $\langle\cdot, \cdot\rangle_{1}$ on $\Omega_{1}(\mathcal{A})$ to a 
scalar product on $\mathscr{E} \otimes_{\mathcal{A}} \Omega_{1}(\mathcal{A})$. This will allow us to construct the kinetic term out of $\nabla \phi$. Let us construct this scalar product on $\mathcal{E}_{\otimes_{\mathcal{A}}} \Omega_{n}(\mathcal{A})$ for any $n \in \mathbb{N}$. For $\Phi, \Psi \in \mathcal{E} \otimes_{\mathcal{A}} \Omega_{n}(\mathcal{A})$, we write

$$
\Phi=\sum_{i} \phi_{i} \otimes \omega_{i}, \quad \Psi=\sum_{i} \psi_{i} \otimes \eta_{i},
$$

with $\phi_{i}, \psi_{i} \in \mathcal{E}$ and $\omega_{i}, \eta_{i} \in \Omega_{n}(\mathcal{A})$. Then one can define the scalar product $\langle\cdot, \cdot\rangle_{\mathcal{A}, n}$ using both $\langle\cdot, \cdot\rangle_{n}$ and $(\cdot, \cdot)_{\mathcal{A}}$ :

$$
\langle\Phi, \Psi\rangle_{\mathcal{A}, n}:=\sum_{i, j}\left\langle\omega_{i},\left(\phi_{i}, \psi_{j}\right)_{\mathcal{A}} \eta_{j}\right\rangle_{n} .
$$

In particular, it allows to define the kinetic term as

$$
\langle\nabla \phi, \nabla \phi\rangle_{\mathcal{A}, 1} \text {. }
$$

To construct the mass term, we simply use the scalar product (2.13):

$$
\mu_{0}^{2}\langle\phi, \phi\rangle_{\mathcal{E}}=\mu_{0}^{2} \operatorname{Tr}_{\mathcal{A}}\left[(\phi, \phi)_{\mathcal{A}}\right] .
$$

For the interaction term, which should be a polynomial in the field, we can extend the former construction. For instance, the basic $\lambda \phi^{4}$ interaction reads

$$
\frac{\lambda}{2} \operatorname{Tr}_{\mathcal{A}}\left[(\phi, \phi)_{\mathcal{A}}^{2}\right]
$$

More generally, one can construct arbitrary monomials

$$
\frac{\lambda}{n} \operatorname{Tr}_{\mathcal{A}}\left[(\phi, \phi)_{\mathcal{A}}^{n}\right]
$$

We may also consider products of such terms. Despite they look very unnatural from the point of view of ordinary QFT, they should be needed to obtain a stable quantum theory; stable with respect to the renormalisation group flow. We will see in the next sections that for a $\phi_{4}^{4}$-theory on the Heisenberg module such a 'product of traces' term is precisely the missing term which will allow us to cure the UV/IR-mixing problem.

The basic action we shall use in the sequel reads

$$
S\left[\phi, \phi^{\dagger}\right]:=\langle\nabla \phi, \nabla \phi\rangle_{\mathcal{A}, 1}+\mu_{0}^{2} \operatorname{Tr}_{\mathcal{A}}\left[(\phi, \phi)_{\mathcal{A}}\right]+\frac{\lambda}{2} \operatorname{Tr}_{\mathcal{A}}\left[(\phi, \phi)_{\mathcal{A}}^{2}\right]
$$

Here we have followed the traditional notation of QFT where a complex field $\phi$ and its conjugate $\phi^{\dagger}$ are treated as independent variables. In our setting $\phi^{\dagger}$ has to be considered as an element of the dual module $\mathcal{E}^{*}$ which consists of $\mathcal{A}$-linear forms on $\&$ defined by

$$
\phi^{\dagger}(\chi):=(\phi, \chi)_{\mathcal{A}}
$$

for any $\chi \in \mathcal{A}$. This distinction between $\phi$ and $\phi^{\dagger}$ will prove to be very convenient when developing the perturbative path integral approach, for instance in getting the 
right symmetry factors. However, it is important to notice that $\mathcal{E}^{*}$ is not the topological dual of $\mathcal{E}$. Moreover, it should be clear that $\mathcal{E}^{*} \simeq \mathcal{E}$ as a linear space, the identification being given by $\phi \leftrightarrow(\phi, \cdot)_{\mathcal{A}}^{*}=(\cdot, \phi)_{\mathcal{A}}$. Also $\mathcal{E}^{*}$ carries the same Fréchet topology as $\varepsilon$.

In the case of a field theory constructed out of a spectral triple, we would like to stress that the choice of the order $n$ of the monomials (2.19) involved in the interaction term depends on the spectral dimension $d$ of the triple. In the general setting, $n$ should be related to the spectral properties of the propagator which is the bounded operator on $\mathscr{H}_{\mathcal{E}}$ given by the inverse of the (densely defined) positive operator $H$ corresponding to the quadratic part of the action (2.20). More precisely, $H$ is defined in terms of the following quadratic form on $\varepsilon$

$$
\langle\phi, H \chi\rangle_{\mathcal{E}}:=\langle\nabla \phi, \nabla \chi\rangle_{\mathcal{A}, 1}, \quad \phi, \chi \in \mathcal{E} \subset \mathscr{H}_{\mathcal{E}}
$$

It is precisely because we are going to study a model coming from a spectral triple of spectral dimension 4 that we focus on a quartic interaction.

It may seem to be quite restrictive to ask for the existence of a faithful trace to define the classical action. Faithfulness is required in order to guarantee that the quantum theory has a power-counting properly related to the spectral properties of the propagator of the model. But traciality is not needed at all, it simply makes the noncommutative models closer to the commutative one. For instance, there are numerous noncommutative algebras giving rise to non-trivial spectral triples (e.g., $\mathrm{SU}_{q}(2)$ [14]) that do not posses any faithful trace. In such circumstance one can define the classical theory by replacing everywhere the trace $\operatorname{Tr}_{\mathcal{A}}$ by a faithful state $\Psi$ on $\mathcal{A}$.

It is also worthwhile to notice that this theory is naturally coupled to gauge fields since it is invariant under

$$
\begin{aligned}
& \phi \rightarrow u \phi \\
& \nabla \rightarrow\left(u \otimes 1_{\Omega_{1}(\mathcal{A})}\right) \nabla u^{-1}
\end{aligned}
$$

where $u$ is a unitary element of the algebra $\mathscr{B}:=\operatorname{End}_{\mathcal{A}}(\mathscr{E})$ of $\mathcal{A}$-linear transformations of $\mathcal{E}$. Thus the gauge invariant action for the field $\phi$ coupled to a Yang-Mills connection $\nabla$ reads

$$
S\left[\phi, \phi^{\dagger}, \nabla\right]:=\langle\mathrm{F}, \mathrm{F}\rangle_{2}+\langle\nabla \phi, \nabla \phi\rangle_{\mathcal{A}, 1}+\mu_{0}^{2} \operatorname{Tr}_{\mathcal{A}}\left[(\phi, \phi)_{\mathcal{A}}\right]+\frac{\lambda}{2} \operatorname{Tr}_{\mathcal{A}}\left[(\phi, \phi)_{\mathcal{A}}^{2}\right],
$$

which involves the scalar product $\langle\cdot, \cdot\rangle_{2}$ on the the space of 2 -forms $\Omega_{2}(\mathcal{A})$. As already mentioned, in the context of spectral triple the curvature is in principle an equivalence class of 2 -forms (modulo the junk ideal). However, we still ignore this point since we are not going to include a gauge degree of freedom when developing the quantum theory. For that reason it is preferable to stick to the action functional (2.20). 


\subsection{Noncommutative tori}

2.2.1. Geometric structures. Let us now work out the previous construction in the case of a $d$-dimensional smooth noncommutative torus. The latter is defined through its algebra of coordinates, $\mathcal{A}_{\Theta}$, which is the algebra of all power series $a=\sum_{\gamma \in \mathbb{Z}^{d}} a_{\gamma} U_{\gamma}$, with fast decreasing coefficients $\left\{a_{\gamma}\right\} \in \mathcal{S}\left(\mathbb{Z}^{d}\right)$. Here $U_{\gamma}$ are unitary elements of $\mathcal{A}_{\Theta}$ fulfilling

$$
U_{\gamma} U_{\gamma^{\prime}}=e^{-i \pi \Theta\left(\gamma, \gamma^{\prime}\right)} U_{\gamma+\gamma^{\prime}}
$$

where $\Theta$ is a skew-symmetric real $d \times d$ matrix which defines a 2-cocycle on the group $\mathbb{Z}^{d} \subset \mathbb{R}^{d}$. The algebra $\mathcal{A}_{\Theta}$ is endowed with its natural Fréchet topology, coming from the set of semi-norms $p_{n}(a)=\sup _{\gamma \in \mathbb{Z}^{d}}\left(\left(1+|\gamma|^{2}\right)^{n}\left|a_{\gamma}\right|\right), n \in \mathbb{N}$, $a \in \mathcal{A}_{\Theta}$. In analogy with its commutative counterpart, where the unitaries $U_{\gamma}$ are nothing but the Fourier modes, the smoothness condition relies on the rapid decay of the sequence $\left\{a_{\gamma}\right\}$.

The structure of this algebra depends on the arithmetical properties of the entries of the matrix $\Theta$. Three typical cases have to be distinguished.

- If all the entries of $\Theta$ are integers, then the algebra $\mathcal{A}_{\Theta}$ is commutative and can be identified with the algebra of smooth functions on an ordinary torus.

- If all the entries of $\Theta$ are rational numbers, then $\mathcal{A}_{\Theta}$ can be realised as a bundle of matrix algebras over an ordinary torus. This is clear for $d=2$, where the unique parameter $\theta=p / q$ in $\Theta$ determines the size of the matrices to be $q$. The general case follows from reducing the matrix $\Theta$ to a direct sum of $2 \times 2$ matrices plus a zero matrix (which corresponds to the null space of $\Theta$ ), using a transformation in $\operatorname{SL}(d, \mathbb{Z})$. The algebra of functions over the underlying torus is nothing but the centre of $\mathcal{A}_{\Theta}$.

- If all the entries of $\Theta$ are irrational numbers and $\Theta$ is invertible, then the centre of the algebra is trivial and $\mathcal{A}_{\Theta}$ is a noncommutative space that cannot be reduced to an ordinary space.

In our analysis of noncommutative field theories we are mostly interested in the irrational case which exhibits some new phenomena. However, it is also interesting to keep in mind the first two cases since they can always be understood in the context of commutative field theories with matrix-valued fields. In the discussion of the non-planar diagrams it will be necessary to single out irrational numbers that fulfil a Diophantine condition. The latter are irrational numbers that are 'far away from the rationals'. The use of the Diophantine condition is not infrequent in noncommutative geometry. For instance, it appears to be crucial in the computation of the Hochschild cohomology of $\mathcal{A}_{\Theta}$ [9], as well as for the heat-invariants [17].

The differential algebra can be constructed out of the $d$ commuting derivations

$$
\delta_{\mu}\left(U_{\gamma}\right):=2 i \pi \gamma_{\mu} U_{\gamma}, \quad \gamma=\left(\gamma_{1}, \ldots, \gamma_{d}\right) \in \mathbb{Z}^{d},
$$


which is the infinitesimal form of the proper action of $\mathbb{T}^{d}$ on $\mathcal{A}_{\Theta}$ given by

$$
\left(e^{2 i \pi \alpha_{1}}, \ldots, e^{2 i \pi \alpha_{d}}\right) \cdot U(\gamma):=e^{2 i \pi \alpha \cdot \gamma} U(\gamma) .
$$

Elements of $\Omega_{n}\left(\mathcal{A}_{\Theta}\right)$ are completely antisymmetric multiplets $\omega_{\mu_{1}, \ldots, \mu_{n}}$ of $\mathcal{A}_{\Theta}$. The multiplication and the differential in $\Omega\left(\mathcal{A}_{\Theta}\right)$ obey the same algebraic rules as the wedge product and the de Rham differential. The algebra $\mathcal{A}_{\Theta}$ has a faithful trace $\operatorname{Tr}_{\mathcal{A}_{\Theta}}$ defined by

$$
\operatorname{Tr}_{\mathcal{A} \Theta}\left(\sum_{\gamma \in \mathbb{Z}^{d}} a_{\gamma} U_{\gamma}\right):=a_{0} .
$$

If $\mathcal{A}_{\Theta}$ is commutative, this trace is nothing but the integral over the underlying torus, with a volume normalised to 1 . In the irrational case, $\operatorname{Tr}_{\mathcal{A} \Theta}$ is the unique faithful trace up to normalisation. The scalar product on $\Omega_{n}\left(\mathcal{A}_{\Theta}\right)$ is made out of the trace

$$
\langle\omega, \eta\rangle_{n}:=\operatorname{Tr}_{\mathcal{A}_{\Theta}}\left(\omega_{\mu_{1} \ldots \mu_{n}}^{*} \eta^{\mu_{1}, \ldots, \mu_{n}}\right),
$$

where the euclidian metric and Einstein's summation convention have been used. This construction follows readily from the general principles, using the spectral triple $\left(\mathcal{A}_{\Theta}, \mathcal{H}, \mathscr{D}\right)$ where $\mathscr{H}=A_{\Theta}^{2^{[d / 2]}}$ with $A_{\Theta}$ the completion of $\mathcal{A}_{\Theta}$ with respect to the norm induced by the scalar product $\langle a, b\rangle_{\mathcal{A}_{\Theta}}:=\operatorname{Tr}_{\mathcal{A}_{\Theta}}\left(a^{*} b\right)$, and where $\mathscr{D}=i \Gamma^{\mu} \delta_{\mu}$ is the standard euclidian Dirac operator. Note that a noncommutative torus is a noncommutative manifold without boundary, in the sense that the integral of a derivative always vanishes: $\operatorname{Tr}_{\mathcal{A} \Theta}\left(\delta_{\mu} U_{\gamma}\right)=0$. This relation is particularly useful in the study of classical field theories because it allows to derive classical field equations and invariance laws.

2.2.2. Heisenberg modules. A wide class of projective modules over $\mathcal{A}_{\Theta}$ can be constructed as projective representations of groups of the type $G \times \hat{G}$ with $G=\mathbb{R}^{p} \times \mathbb{Z}^{q} \times F$, where $F$ is a finite Abelian group and $\widehat{G}$ the dual of $G$ [25]. Equivalently, these projective representations can be thought of as representations of the Heisenberg groups associated to the corresponding central extensions. The group $G \times \widehat{G}$ acts by unitary operators on the Hilbert space $L^{2}(G, d g)$ obtained by completion of the space of smooth fast decreasing functions $\delta(G)$ on $G$. For $(g, \mu) \in G \times \widehat{G}$ and $\psi \in L^{2}(G, d g)$, the action is

$$
T_{g, \mu} \psi(x):=\mu(g)^{1 / 2} \mu(x) \psi(x+g) .
$$

Then, given a lattice $\Gamma$ isomorphic to $\mathbb{Z}^{d}$ in $G \times \widehat{G}$, one can represent the algebra $\mathcal{A}_{\Theta}$ (acting on the right) on $L^{2}(G, d g)$ by restricting the action (2.30) to the subgroup $\Gamma$ :

$$
\phi U_{\gamma}:=T_{g, \mu} \phi, \quad \phi \in L^{2}(G, d g), \gamma=(g, \mu) \in \Gamma .
$$

The multiplication law of the algebra $\mathcal{A}_{\Theta}$ is satisfied with respect to the so-called 
Heisenberg cocycle: $e^{-2 i \pi \Theta\left(\gamma, \gamma^{\prime}\right)}:=\mu\left(g^{\prime}\right) \mu^{\prime}(g)^{-1}$. If we assume that $(G \times \widehat{G}) / \Gamma$ is compact, then $\mathscr{E}_{8}:=\varnothing(G)$ is a finitely generated projective module called the Heisenberg module. In particular, this forces the dimension of the noncommutative torus $d$ to be even, which means that for $a \in \mathcal{A}_{\Theta}$ and $\phi \in \mathcal{E}_{8}, \phi a=\sum_{\gamma} a_{\gamma} \phi U_{\gamma}$ is well defined as an element of $\mathcal{E}_{8}$. This can be proven by elementary Fourier analysis. The module $\mathscr{E}_{\&}$ is equipped with a $\mathcal{A}_{\Theta}$-valued scalar product defined by

$$
(\phi, \chi)_{\mathcal{A}_{\Theta}}:=\sum_{\gamma \in \Gamma}\left\langle\phi, \chi U_{\gamma}\right\rangle_{L^{2}(G, d g)} U_{-\gamma} .
$$

This pairing takes values in the smooth algebra $\mathcal{A}_{\Theta}$ and not in its $\mathrm{C}^{*}$-completion. Again basic Fourier analysis shows that the sequence $\left\{\left\langle\phi, \chi U_{\gamma}\right\rangle_{L^{2}(G, d g)}\right\}_{\gamma \in \mathbb{Z}^{d}}$ is of Schwartz class whenever $\phi, \chi \in \varepsilon_{8}$.

A connection on $\mathscr{E}_{\&}$ is entirely specified by its covariant derivatives $\nabla_{\mu}$ once we have identified $\Omega_{1}\left(\mathcal{A}_{\Theta}\right)$ with $\left(\mathscr{A}_{\Theta}\right)^{d}$. The canonical Heisenberg connection $\nabla$ is obtained from the infinitesimal action of the continuous part of the group $G \times \widehat{G}$. In general, it both involves partial derivatives $\frac{\partial}{\partial x_{i}}$ (action of $\mathbb{R}^{p}$ ) and multiplication by $x_{i}$ (action of $\left.\left(\mathbb{R}^{p}\right)^{*}\right)$. Finally, it is also useful to note that the endomorphism algebra End $_{\mathcal{A}_{\Theta}}\left(\mathcal{E}_{\&}\right)$ is nothing but another noncommutative torus generated by the dual lattice $\widehat{\Gamma} \in \widehat{G} \times G$ which pairs trivially with $\Gamma$ with respect to the Heisenberg cocycle.

Besides, the curvature of $\nabla$ is defined as $\mathrm{F}_{\mu \nu}:=\left[\nabla_{\mu}, \nabla_{\nu}\right]$, which is always an anti-hermitian element of End $\mathcal{A}_{\Theta}\left(\varepsilon_{\&}\right)$.

To construct the action functional for a classical field $\phi \in \mathcal{E}_{\S}$, we choose a constant positive definite matrix $g_{\mu \nu}$ to define a scalar product between 1-forms so that the general form of the action given by (2.20) is

$$
\begin{aligned}
S[\phi, \bar{\phi}]= & \sqrt{g} g^{\mu \nu} \operatorname{Tr}_{\mathcal{A}_{\Theta}}\left[\left(\nabla_{\mu} \phi, \nabla_{\nu} \phi\right)_{\mathcal{A}_{\Theta}}\right] \\
& +\sqrt{g} \mu_{0}^{2} \operatorname{Tr}_{\mathcal{A}_{\Theta}}\left[(\phi, \phi)_{\mathcal{A}_{\Theta}}\right]+\sqrt{g} \frac{\lambda}{2} \operatorname{Tr}_{\mathcal{A}_{\Theta}}\left[(\phi, \phi)_{\mathcal{A}_{\Theta}}^{2}\right]
\end{aligned}
$$

with $g^{\mu \nu}$ denoting the inverse of $g_{\mu \nu}$ and $g$ its determinant. Since the module we consider is made out of complex-valued functions, from now on we denote an element of the dual module $\varepsilon_{\&}^{*}$ by $\bar{\phi}$. Note that more general terms such as

$$
\left[\operatorname{Tr}_{\mathcal{A} \Theta}\left[(\phi, \phi)_{\mathcal{A} \Theta}\right]^{n}\right]^{k}
$$

can be introduced. In the last section, we shall see that for $d=4$ the renormalisation forces the introduction of such a term with $n=1, k=2$.

The simplest example of a Heisenberg module is constructed explicitly for $d=2$ as follows. In the two-dimensional case there is only one deformation parameter in the matrix $\Theta$, namely $\theta$. We will denote by $\mathcal{A}_{\theta}$ the corresponding noncommutative torus algebra. In this case we start with the projective representation $(2.30)$ of $\mathbb{R} \times(\mathbb{R})^{*}$ on 
the Schwartz space of the real line. Then one defines the lattice $\Gamma$ and its dual $\widehat{\Gamma}$ as

$$
\begin{aligned}
& \Gamma:=\{\gamma=(\theta m, 2 \pi n) \text { with } m, n \in \mathbb{Z}\}, \\
& \hat{\Gamma}:=\left\{\hat{\gamma}=\left(m^{\prime}, \frac{2 \pi n^{\prime}}{\theta}\right) \text { with } m^{\prime}, n^{\prime} \in \mathbb{Z}\right\},
\end{aligned}
$$

with $\theta>0$. The lattices $\Gamma$ and $\widehat{\Gamma}$ define two commuting noncommutative torus algebras $\mathscr{A}_{\theta}$ and $\operatorname{End}_{\mathcal{A}_{\theta}}\left(\mathscr{E}_{8}\right)=\mathcal{A}_{1 / \theta}$ acting on $\phi \in \mathscr{E}_{8}$ as

$$
\begin{aligned}
& \left(\phi U_{\gamma}\right)(x):=e^{i \pi \theta m n} e^{2 i \pi n x} \phi(x+m \theta), \\
& \left(U_{\hat{\gamma}} \phi\right)(x):=e^{i \frac{\pi m n}{\theta}} e^{\frac{2 i \pi n^{\prime} x}{\theta}} \phi\left(x+m^{\prime}\right) .
\end{aligned}
$$

In the previous equation we have identified $\left(\mathbb{R} \times \mathbb{R}^{*}\right)^{*}=\mathbb{R}^{*} \times \mathbb{R}$ with $\mathbb{R} \times \mathbb{R}^{*}$ so that the lattice and its dual are both subsets of $\mathbb{R} \times \mathbb{R}^{*}$.

The $\mathcal{A}_{\theta}$-valued scalar product follows from the general form (2.32) and is explicitly given by

$$
(\phi, \chi)_{\mathcal{A}_{\theta}}=\sum_{\gamma \in \Gamma}\left(e^{i \pi \theta m n} \int_{\mathbb{R}} d x \bar{\phi}(x) e^{2 i \pi n x} \chi(x+m \theta)\right) U_{-\gamma} .
$$

The Heisenberg connection is given by the two covariant derivatives

$$
\nabla_{1} \phi(x)=-\frac{2 i \pi x}{\theta} \phi(x) \quad \text { and } \quad \nabla_{2} \phi(x)=\frac{d \phi(x)}{d x} .
$$

This connection minimise the Yang-Mills action [13] and has a constant curvature given by

$$
\mathrm{F}_{12}=\left[\nabla_{1}, \nabla_{2}\right]=\frac{2 i \pi}{\theta}
$$

In this example, with the euclidian metric $g_{\mu \nu}=\delta_{\mu \nu}$, the action functional (2.33) reads

$$
\begin{aligned}
S[\phi, \bar{\phi}]:= & \int_{\mathbb{R}} d x \bar{\phi}(x)\left(-\frac{d^{2}}{d x^{2}}+\frac{4 \pi^{2}}{\theta^{2}} x^{2}\right) \phi(x)+\mu_{0}^{2} \int_{\mathbb{R}} d x \bar{\phi}(x) \phi(x) \\
& +\frac{\lambda}{2} \sum_{m, n \in \mathbb{Z}} \int_{\mathbb{R}} d x \bar{\phi}(x+n+m \theta) \phi(x+n) \bar{\phi}(x) \phi(x+m \theta) .
\end{aligned}
$$

To derive this expression from (2.37), we used the Poisson re-summation formula in the sense of tempered distributions to write $\sum e^{2 i \pi n x}=\sum \delta(x+n)$. The kinetic part of this action is simply the energy of an harmonic oscillator. The interaction takes a non-local form in $x$-space because of the summation over $m$ and $n$, but reduces to the an-harmonic oscillator for $m=n=0$. We shall further comment on the non-local structure of this interaction in Section 3.3.1 once we have derived the Feynman rules. 
So far we have seen that for the Heisenberg module there are two notions of dimension. The first one, $d$, is the spectral dimension of the noncommutative torus whereas the second, $d / 2$, is the dimension of the representation space entering in $\varepsilon_{\S}\left(\mathbb{R}^{d / 2}\right)=\delta\left(\mathbb{R}^{d / 2}\right)$. In the sequel, in view of quantum field application, we will see that the pertinent notion of dimension is that of the noncommutative torus. In the following and unless otherwise specified, $d$ will always denote the dimension of the noncommutative torus, which moreover has to be even.

2.2.3. Symmetries and field equation. We are now going to review the classical discrete symmetries of the model. First note that this action is invariant under a version of the Langmann-Szabo duality [24]. If we replace $\phi$ by its Fourier transform

$$
\eta(\xi)=\int_{\mathbb{R}} d x e^{-2 i \pi x \xi} \phi(x),
$$

then the action is invariant, up to a rescaling of the different parameters:

$$
S_{\lambda, \mu_{0}, \theta}[\phi, \bar{\phi}]=\frac{1}{\theta^{2}} S_{\theta \lambda, \theta^{2} \mu_{0}, 1 / \theta}[\eta, \bar{\eta}] .
$$

This duality is an essential tool in the study of the renormalisability of the model.

Besides the Langmann-Szabo duality, there is another discrete symmetry involving the Fourier transform. This symmetry is related to the action of the modular group $\operatorname{SL}(2, \mathbb{Z})$ on the modulus $\tau$ in the upper half plane. Here $\tau$ parameterises the constant matrix of determinant one, entering in the kinetic term as

$$
g=\frac{1}{\operatorname{Im}(\tau)}\left(\begin{array}{cc}
1 & \operatorname{Re}(\tau) \\
\operatorname{Re}(\tau) & |\tau|^{2}
\end{array}\right)
$$

Then the action is invariant under simultaneous changes of the field and the modulus under the generators $S$ and $T$ of the modular group,

$$
\begin{aligned}
& S: \phi(x) \rightarrow \frac{1}{\sqrt{\theta}} \int_{\mathbb{R}} d \xi e^{\frac{-2 i \pi x \xi}{\theta}} \phi(\xi), \quad \tau \rightarrow-\frac{1}{\tau}, \\
& T: \phi(x) \rightarrow e^{\frac{-i \pi x^{2}}{\theta}} \phi(x), \quad \tau \rightarrow \tau+1 .
\end{aligned}
$$

For such a metric the Langmann-Szabo duality must also be accompanied by the action of $S$ on the modulus. The transformations given by $S$ and $T$ define outer automorphisms of the algebra of the noncommutative torus and correspond to large diffeomorphisms of the torus. Other outer automorphisms are given by the translations defined in (2.27), but they do not leave the action invariant because of the non-trivial connection. Translation invariance can only be recovered by including gauge fields.

We have displayed the construction in the two-dimensional case with the simplest Heisenberg module. More general modules can easily be obtained in any even dimension by simply tensoring this module by itself $d / 2$ times. In this case the action takes 
the same form as in (2.40) with $x, m$ and $n$ replaced by $d / 2$-dimensional vectors. The Langmann-Szabo duality takes the same form as before except that the coupling constant $\lambda$ transforms as

$$
\lambda \rightarrow \lambda \theta^{2-\frac{d}{2}} .
$$

In particular, the coupling constant is invariant in four dimensions.

It is also interesting to look at the classical field equation

$$
\frac{\delta S[\phi, \bar{\phi}]}{\delta \bar{\phi}}=0, \quad \frac{\delta S[\phi, \bar{\phi}]}{\delta \phi}=0,
$$

where, as usual, the functional derivatives are defined in the weak sense, with respect to the pairing (2.13). Because the action is "symmetric" in $\phi$ and $\bar{\phi}$, it is sufficient to look at one of them. The first one reads

$$
H \phi=-\lambda \phi(\phi, \phi)_{\mathcal{A}_{\theta}}=-\lambda \sum_{\gamma \in \Gamma}\left\langle\phi, \phi U_{\gamma}\right\rangle_{L^{2}(\mathbb{R})} \phi U_{-\gamma},
$$

where

$$
H:=-\frac{d^{2}}{d x^{2}}+\frac{4 \pi^{2}}{\theta^{2}} x^{2}+\mu_{0}^{2}
$$

is the harmonic oscillator Hamiltonian with frequency $2 \pi / \theta$. In the explicit realisation of the module $\varepsilon_{s}=\delta(\mathbb{R})$ the field equation can be rewritten as

$$
\left(-\frac{d^{2}}{d x^{2}}+\frac{4 \pi^{2}}{\theta^{2}} x^{2}+\mu_{0}^{2}\right) \phi(x)=-\lambda \sum_{n, m \in \mathbb{Z}} \phi(x+n) \bar{\phi}(x+n+m \theta) \phi(x+m \theta) .
$$

A very important task would be to study the solutions of the classical field equation.

2.3. Holomorphic representation. For our purposes it is convenient to work with an equivalent representation of the Heisenberg modules in terms of holomorphic functions. In the general case these are constructed out of the space of square integrable holomorphic functions on $\mathbb{C}$ with respect to the scalar product

$$
\langle\phi, \chi\rangle_{B}:=\int d \mu(z, \bar{z}) \bar{\phi}(\bar{z}) \chi(z)
$$

with the Gaußian measure normalised as $d \mu(z, \bar{z}):=(\omega / \pi) e^{-\omega|z|^{2}} d \operatorname{Re}(z) d \operatorname{Im}(z)$ and $\omega>0$ an arbitrary parameter. We denote by $\mathscr{H}_{B}:=L_{\text {hol }}^{2}(\mathbb{C}, d \mu)$ the corresponding Hilbert space. It also admits a projective representation of $\mathbb{C} \simeq \mathbb{R} \times \mathbb{R}^{*}$ given by

$$
\left(T_{v} \phi\right)(z):=e^{-\frac{\omega|v|^{2}}{2}-\omega \bar{v} z} \phi(z+v), \quad v \in \mathbb{C} .
$$


The operators $T_{v}$ are unitary with respect to the scalar product $\langle\cdot, \cdot\rangle_{B}$ and obey the multiplication rule

$$
T_{v} T_{w}=e^{\frac{\omega}{2}(\bar{v} w-\bar{w} v)} T_{v+w} .
$$

Let us choose a square lattice in $\mathbb{C}$ parametrised by $l>0$,

$$
\Gamma:=\left\{\gamma=l(m+i n) \text { with }(m, n) \in \mathbb{Z}^{2}\right\} .
$$

One can define a right action $U_{\gamma}$ of $\mathcal{A}_{\theta}$, with $\theta=\frac{\omega l^{2}}{\pi}$, by restricting the action (2.51) to the lattice $\Gamma$, i.e., $\phi U_{\gamma}:=T_{\gamma} \phi, \gamma \in \Gamma$. Here and in the following we make a slight abuse of notation by denoting by $U_{\gamma}$ an element of $\mathcal{A}_{\theta}$ as well as the operator acting on the right on $\mathscr{H}_{B}$. Note that in this framework the dual lattice is given by

$$
\widehat{\Gamma}:=\left\{\hat{\gamma}=\frac{\pi}{\omega l}\left(m^{\prime}+i n^{\prime}\right) \text { with }\left(m^{\prime}, n^{\prime}\right) \in \mathbb{Z}^{2}\right\}
$$

and gives rise to an action of $\mathcal{A}_{1 / \theta}$. The scalar product with values in the algebra $\mathcal{A}_{\theta}$ is defined as in (2.32), with $\langle\cdot, \cdot\rangle_{B}$ denoting the Bargmann scalar product:

$$
(\phi, \chi)_{\mathcal{A}_{\theta}}=\sum_{\gamma \in \Gamma}\left\langle\phi, \chi U_{\gamma}\right\rangle_{B} U_{-\gamma} .
$$

The connection follows from the infinitesimal action of the translation group on $\mathscr{H}_{B}$. It reads

$$
\nabla_{1} \phi(z)=\frac{\pi}{i \omega l R}\left[\frac{d \phi}{d z}(z)+\omega z \phi(z)\right] \text { and } \nabla_{2} \phi(z)=\frac{\pi}{\omega l R}\left[\frac{d \phi}{d z}(z)-\omega z \phi(z)\right]
$$

and of course they fulfil the Leibniz rule $\nabla_{i}\left(\phi U_{\gamma}\right)=\left(\nabla_{i} \phi\right) U_{\gamma}+\phi\left(\delta_{i} U_{\gamma}\right), i=1,2$, where the derivations of $\mathcal{A}_{\theta}$ have been defined in (2.26). Note that we have introduced an extra parameter $R>0$ which has the dimension of a length and has to be thought of as the 'radius' of the noncommutative torus. It is to be noted that this connection has constant curvature $\mathrm{F}_{12}=\left[\nabla_{1}, \nabla_{2}\right]=\frac{2 i \pi^{2}}{\omega l^{2} R^{2}}$ and that $\operatorname{Tr}_{\mathcal{A}_{\theta}}\left[\mathrm{F}_{12}\right]=2 i \pi \frac{\pi}{\omega l^{2}}$. This provides a topological invariant of the bundle analogous to the first Chern class. Usually one includes the factor $\frac{\pi}{\omega l^{2}}$ in the trace since it corresponds to the dimension of the bundle [12]. Therefore the topological invariant belongs to $2 i \pi \mathbb{Z}$, which holds for arbitrary projective modules and arbitrary connections over a two-dimensional noncommutative torus.

From a physical point of view the Bargmann module appears in the analysis of the motion of an electron confined to the $x y$-plane in an external uniform magnetic field of strength $B$ along an orthogonal axis. If we denote by $-e$ and $m$ the charge and the mass of the electron, the Hamiltonian is, in the Landau gauge,

$$
H_{\mathrm{L}}=-\frac{\hbar^{2}}{2 m}\left[\left(\frac{\partial}{\partial x}-\frac{i e B}{2 \hbar} y\right)^{2}+\left(\frac{\partial}{\partial y}+\frac{i e B}{2 \hbar} x\right)^{2}\right] \text {. }
$$


Translation invariance is realised through the magnetic translation operators

$$
\left(T_{a, b} \psi\right)(x, y):=e^{\frac{i e B}{2 \hbar}(a y-b x)} \psi(x+a, y+b),
$$

which form a projective representation of the translation group,

$$
T_{a, b} T_{a^{\prime}, b^{\prime}}=e^{\frac{i e B}{2 \hbar}\left(a b^{\prime}-b a^{\prime}\right)} T_{a+a^{\prime}, b+b^{\prime}} .
$$

Let us now assume that the magnetic field is strong enough so that the electron is confined to the lowest Landau level, which is the ground state of the Hamiltonian $H_{\mathrm{L}}$ with energy $\frac{\hbar e B}{2 m}$.

The wave functions pertaining to the lowest Landau level are conveniently written using a complex coordinate $z=x+i y$,

$$
\psi(z, \bar{z})=e^{-\frac{e B}{4 \hbar}|z|^{2}} \phi(z),
$$

where $\phi$ is an arbitrary holomorphic function with square summable Taylor coefficients. Thus, the lowest Landau level is infinitely degenerated and its wave functions are in one-to-one correspondence with analytic functions $\phi$ in the Bargmann space $\mathscr{H}_{B}$ with $\omega=\frac{e B}{2 \hbar}$. Besides, a magnetic translation by $v=a+i b$ acts on $\phi$ via the action of $\mathbb{C}$ on holomorphic functions (2.51).

The noncommutative tori arise naturally when we consider the effect of an atomic lattice in perturbation theory. Indeed, let us assume that in addition to the magnetic field the electron is submitted to a potential $V$ created by a square lattice of spacing $l$. Then the potential is $\mathbb{Z}^{2}$-periodic, $V(x+l m, y+n l)=V(x, y)$ for any $(m, n) \in \mathbb{Z}^{2}$. Furthermore, let us make the assumption that the magnetic field is strong enough so that one can consider the electron to be confined to the lowest Landau level even in the presence of $V$. Therefore the implementation of the lattice translations implies that the lowest Landau level is nothing but the Bargmann module $\mathscr{H}_{B}$ over the noncommutative torus $\mathcal{A}_{\theta}$ with a parameter

$$
\theta=\frac{\omega l^{2}}{\pi}=\frac{e B l^{2}}{2 \pi \hbar}=\frac{\Phi}{\Phi_{0}}
$$

where $\Phi=B l^{2}$ is the flux of $B$ through the unit cell of the lattice and $\Phi_{0}=\frac{2 \pi \hbar}{e}$ is the quantum flux. This noncommutative torus may be seen as a Noncommutative Brillouin zone since it replaces the ordinary Brillouin zone in the presence of the magnetic field. For this interpretation to hold it is necessary to take $R=\frac{2 \pi}{l}$, which corresponds to the size of the Brillouin zone. Let us note that the topological invariant given by $\operatorname{Tr}_{\mathcal{A}_{\Theta}}\left(F_{12}\right)$ is nothing but the Hall conductivity [2].

If the electron remains confined to the lowest Landau level, then $V$ has to be projected on $\mathscr{H}_{B}$ and lattice invariance translates into the statement that $V$ must commute with the left action of $\mathcal{A}_{\theta}$. Therefore, $V$ is an element of the algebra $\mathcal{A}_{1 / \theta}$ 
acting on the right. This is a general constraint on the action of $V$, solely derived from symmetry considerations and independent of the precise form of $V$.

Obviously, the projective modules constructed out of the Schwartz space and the Bargmann space are equivalent, the explicit equivalence being the unitary Bargmann transform $B: \mathscr{H}_{S} \rightarrow \mathscr{H}_{\mathscr{B}}$ defined as

$$
(B \chi)(z)=\left(\frac{\omega}{\pi}\right)^{1 / 2} \int_{\mathbb{R}} d x e^{-\frac{\omega\left(x^{2}+z^{2}\right)}{2}+\sqrt{2} \omega z x} \chi(x),
$$

and its inverse is

$$
\left(B^{-1} \phi\right)(x)=\left(\frac{\omega}{\pi}\right)^{1 / 2} \int_{\mathbb{C}} d \mu(z, \bar{z}) e^{-\frac{\omega\left(x^{2}+\bar{z}^{2}\right)}{2}+\sqrt{2} \omega \bar{z} x} \phi(z)
$$

for $\chi \in L^{2}(\mathbb{R})$ and $\phi \in \mathscr{H}_{B}$. This corresponds to the equivalence between the vertical polarisation on the phase space $\mathbb{R} \times \mathbb{R}^{*}$ and the holomorphic one. Then one defines the smooth module $\mathcal{E}_{B}$ as the image of Schwartz space under the Bargmann transform.

Consider now the projective action $T_{a, b}$ of $\mathbb{R} \times \mathbb{R}^{*}$ on the Schwartz functions of the real line. Its image through the Bargmann transform is an action of $\mathbb{C}$ by the operators $T_{v}$ given in (2.51) with $v=\frac{1}{\sqrt{2}}\left(a+\frac{i b}{\omega}\right)$. This allows to establish a general correspondence between the two projective modules, their scalar products and their connections. In what follows, it is particularly convenient to set $\omega=\frac{2 \pi}{\theta}$ in such a way that the lattices $\Gamma$ and $\widehat{\Gamma}$ read, in the holomorphic formulation,

$$
\Gamma=\left\{\frac{\theta}{\sqrt{2}}(m+i n) \text { with }(m, n) \in \mathbb{Z}^{2}\right\}
$$

and

$$
\widehat{\Gamma}=\left\{\frac{1}{\sqrt{2}}\left(m^{\prime}+i n^{\prime}\right) \text { with }\left(m^{\prime}, n^{\prime}\right) \in \mathbb{Z}^{2}\right\} .
$$

It amounts to set $l=\frac{\theta}{\sqrt{2}}$ in the general square lattice introduced at the beginning of this section. An arbitrary lattice in $\mathbb{C}$ would correspond to an arbitrary lattice in $\mathbb{R} \times \mathbb{R}^{*}$ using the inverse Bargmann transform. From now on we restrict ourselves to the preceding values of $\omega$ and $l$.

In this framework the two operators representing the connection read

$$
\nabla_{1} \phi(z)=\frac{1}{i \sqrt{2}}\left[\frac{d \phi}{d z}(z)+\omega z \phi(z)\right] \text { and } \nabla_{2} \phi(z)=\frac{1}{\sqrt{2}}\left[\frac{d \phi}{d z}(z)-\omega z \phi(z)\right] \text {, }
$$

as follows from (2.56) with $\omega=\frac{2 \pi}{\theta}, l=\frac{\theta}{\sqrt{2}}$ and $R=1$. The operator (2.48) entering into the definition of the kinetic term is

$$
H=-\left(\nabla_{1}\right)^{2}-\left(\nabla_{2}\right)^{2}+\mu_{0}^{2}=2 \omega\left(z \frac{d}{d z}+\frac{1}{2}\right)+\mu_{0}^{2},
$$


which is nothing but the image through the Bargmann transform of the harmonic oscillator Hamiltonian $-\frac{d^{2}}{d x^{2}}+\omega^{2} x^{2}+\mu_{0}^{2}$. Note that there is a constant term in $H$ even if $\mu_{0}=0$. Thus, even in a massless theory there is a priori no infrared divergences at the tree level. Of course, this picture may change at higher loop order because of the IR/UV mixing.

In the development of the quantum theory associated to these projective modules, it will be interesting to have at our disposal higher dimensional analogues of the previous construction. While the general structure of projective modules over higher dimensional noncommutative tori is extremely rich [25], we can easily construct simple examples in any even dimension $d$ by simply taking the $\frac{d}{2}^{\text {th }}$ tensor power of the previous module. This corresponds to a Bargmann space of holomorphic functions on $\mathbb{C}^{d / 2}$. The corresponding lattices are simply obtained by replacing the integers $m$, $n, m^{\prime}$ and $n^{\prime}$ by vectors in $\mathbb{Z}^{\frac{d}{2}}$. This procedure leads to a noncommutative algebra $\mathcal{A}_{\Theta}$ with a $d \times d$ matrix $\Theta$ which is a direct sum of $2 \times 2$ antisymmetric matrices,

$$
\Theta=\left(\begin{array}{rrrrr}
0 & \theta & & & \\
-\theta & 0 & & & \\
& & \ddots & & \\
& & & 0 & \theta \\
& & & -\theta & 0
\end{array}\right) .
$$

To emphasise the difference with a generic noncommutative torus algebra $\mathcal{A}_{\Theta}$, we will denote, as in the two-dimensional case, the algebra corresponding to the specific choice (2.68) by $\mathcal{A}_{\theta}$.

\section{QFT and diagrammatics}

3.1. Feynman rules. In this section, we restore the notation $\phi^{\dagger}$, instead of the complex conjugate notation $\bar{\phi}$, to denote an element of the dual module $\mathcal{E}^{*}$. The reason is that we would like to sketch a representation-free construction of Feynman rules for a field theory on the Heisenberg modules corresponding to the choice (2.68). Otherwise specified, $\mathcal{E}$ can be either $\mathcal{E}_{\&}\left(\mathbb{R}^{d / 2}\right)$ or $\mathcal{E}_{B}\left(\mathbb{C}^{d / 2}\right)$ or even another unitarily equivalent module. We also emphasise that such a procedure does not rely on the particular structures of the module studied so that it can be employed for the construction of any quantum field theory on a projective module.

The action functional $S\left[\phi, \phi^{\dagger}\right]$ we have defined in the previous section can serve as a basis for the construction of a perturbative quantum field theory. This amounts to define and study the $(2 N)$-point functions $G_{2 N}$, given in terms of the functional 
integral

$$
G_{2 N}:=\frac{\int[D \phi]\left[D \phi^{\dagger}\right]\left(\phi \otimes \phi^{\dagger} \otimes \cdots \otimes \phi \otimes \phi^{\dagger}\right) e^{-S\left[\phi, \phi^{\dagger}\right]}}{\int[D \phi]\left[D \phi^{\dagger}\right] e^{-S\left[\phi, \phi^{\dagger}\right]}}
$$

as a perturbation of a free Gaußian functional integral. Because the projective module can be realised as a dense subspace of a Hilbert space of functions with a kinetic term given by a second order differential operator, the free field theory is always well defined. We will always assume in this section that the functional integral has been regularised, for example by integrating only over a finite-dimensional subspace of the projective module. Later on, we shall use other regularisation techniques suitable for renormalisation.

This means that once the theory is regularised, the Green's functions $G_{2 N}$ define

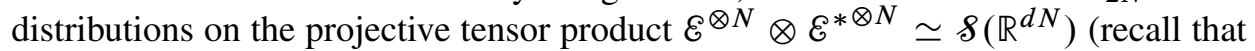
$\delta\left(\mathbb{R}^{d / 2}\right)$ is a nuclear space). The evaluation of $G_{2 N}$ on $2 N$ test functions $\psi_{i} \in \mathcal{E}$, $\chi_{i}^{\dagger} \in \mathcal{E}^{*}, i=1, \ldots, N$, is given by the following path integral formula:

$$
\begin{aligned}
& G_{2 N}\left(\chi_{1}^{\dagger}, \ldots, \chi_{n}^{\dagger}, \psi_{1}, \ldots, \psi_{2}\right) \\
& =\frac{\int[D \phi]\left[D \phi^{\dagger}\right] \operatorname{Tr}_{\mathcal{A}_{\theta}}\left[\left(\chi_{1}, \phi\right)_{\mathcal{A}_{\theta}}\right] \operatorname{Tr}_{\mathcal{A}_{\theta}}\left[\left(\phi, \psi_{1}\right)_{\mathcal{A}_{\theta}}\right] \ldots \operatorname{Tr}_{\mathcal{A}_{\theta}}\left[\left(\chi_{n}, \phi\right)_{\mathcal{A}_{\theta}}\right] \operatorname{Tr}_{\mathcal{A}_{\theta}}\left[\left(\phi, \psi_{n}\right)_{\mathcal{A}_{\theta}}\right] e^{-S\left[\phi, \phi^{\dagger}\right]}}{\int[D \phi]\left[D \phi^{\dagger}\right] e^{-S\left[\phi, \phi^{\dagger}\right]}} .
\end{aligned}
$$

It has to be noted that the field $\phi$ and its conjugate $\phi^{\dagger}$ must occur an equal number of times, as required by the invariance under global phase multiplication. Any correlation function not fulfilling this condition vanishes identically. Note that the field $\phi$ lives in a module $\mathcal{E}$ and its conjugate $\phi^{\dagger}$ in the dual module $\mathcal{E}^{*}$, so that the correlation function with $2 N$ fields is conveniently seen as a linear map from $\varepsilon^{\otimes N}$ to itself.

As usual in QFT, all the correlations functions can be gathered into the generating functional

$$
Z\left[J, J^{\dagger}\right]=\frac{\int[D \phi]\left[D \phi^{\dagger}\right] e^{-S\left[\phi, \phi^{\dagger}\right]+\operatorname{Tr}_{\mathcal{A}_{\theta}}\left[(J, \phi)_{\mathcal{A}_{\theta}}\right]+\operatorname{Tr}_{\mathcal{A}_{\theta}}\left[(\phi, J)_{\mathcal{A}_{\theta}}\right]}}{\int[D \phi]\left[D \phi^{\dagger}\right] e^{-S\left[\phi, \phi^{\dagger}\right]}}
$$

From $Z\left[J, J^{\dagger}\right]$ we define $W\left[J, J^{\dagger}\right]=\log Z\left[J, J^{\dagger}\right]$ and $\Gamma\left[\phi, \varphi^{\dagger}\right]$ as the Legendre transform of $W\left[J, J^{\dagger}\right]$,

$$
\Gamma\left[\varphi, \varphi^{\dagger}\right]=\operatorname{Tr}_{\mathcal{A}_{\theta}}\left[(\varphi, J)_{\mathcal{A}_{\theta}}\right]+\operatorname{Tr}_{\mathcal{A}_{\theta}}\left[(J, \varphi)_{\mathcal{A}_{\theta}}\right]-W\left[J, J^{\dagger}\right]
$$

with $\varphi=\frac{\delta W}{\delta J^{\dagger}}$ and $\varphi^{\dagger}=\frac{\delta W}{\delta J}$. Here $Z$ is obtained as a sum over all Feynman diagrams, $W$ involves only connected diagrams and $\Gamma$ only 1 PI diagrams, i.e., diagrams that 
remain connected if one cuts any internal line. In the sequel, we shall always restrict our analysis to 1PI diagrams.

Since the action functional can be written as

$$
S\left[\phi, \phi^{\dagger}\right]=\operatorname{Tr}_{\mathcal{A}_{\theta}}\left[(\phi, H \phi)_{\mathcal{A}_{\theta}}+\frac{\lambda}{2}(\phi, \phi)_{\mathcal{A}_{\theta}}^{2}\right],
$$

it is convenient to rewrite the quartic interaction using a Hubbard-Stratonovitch transform as

$$
e^{-\frac{\lambda}{2} \operatorname{Tr}_{\mathcal{A}_{\theta}}\left[(\phi, \phi)_{\mathcal{A}_{\theta}}^{2}\right]}=\int[D A] e^{-\operatorname{Tr}_{\mathcal{A}_{\theta}}\left[\frac{\lambda A^{2}}{2}+i \lambda A(\phi, \phi)_{\mathcal{A}_{\theta}}\right]},
$$

where we integrate over all Hermitian elements $A^{*}=A \in \mathcal{A}_{\theta}$. From a physical perspective this amounts to consider $A$ as a random external field subjected to Gaußian probability law.

As usual a regularisation is self-understood and we have dropped an irrelevant normalisation factor. Thus, the correlation function reads

$$
G_{2 N}=\frac{\int[D \phi]\left[D \phi^{\dagger}\right][D A]\left(\phi \otimes \phi^{\dagger} \otimes \cdots \otimes \phi \otimes \phi^{\dagger}\right) e^{-\operatorname{Tr}_{\mathcal{A}_{\theta}}\left[(\phi, H \phi)_{\mathcal{A}_{\theta}}+\frac{\lambda A^{2}}{2}+i \lambda A(\phi, \phi)_{\mathcal{A}_{\theta}}\right]}}{\int[D \phi]\left[D \phi^{\dagger}\right][D A] e^{-\operatorname{Tr}_{\mathcal{A}_{\theta}}\left[(\phi, H \phi)_{\mathcal{A}_{\theta}}+\frac{\lambda A^{2}}{2}+i \lambda A(\phi, \phi)_{\mathcal{A}_{\theta}}\right]}} .
$$

This allows us to derive the Feynman rules in a very simple way. There is a trivial propagator for the $A$-field

$$
\sim \stackrel{\gamma}{\sim} \sim=\frac{1}{\lambda}
$$

Note that the field $A$ appears only in internal lines since we compute only correlations of $\phi$ and $\phi^{\dagger}$. We have also used an explicit expansion over the noncommutative Fourier modes $A=\sum_{\gamma} A_{\gamma} U_{\gamma}$. The fields $\phi$ and $\phi^{\dagger}$ propagate according to

$$
\longrightarrow=H^{-1}
$$

where $H$ is defined in (2.67). Because the fields are complex, the propagator is not symmetric and an orientation on the lines is necessary. We always consider $\phi^{\dagger}$ as incoming and $\phi$ as outgoing, so that the propagator makes sense as a linear map from $\mathcal{E}$ to itself. Finally, the interaction vertex is

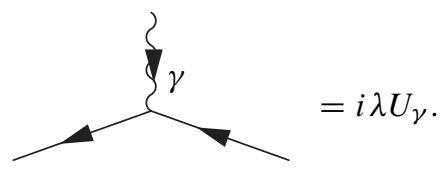

It is important to note that there is a cyclic ordering of the fields $A, \phi^{\dagger}$ and $\phi$ at the vertices which is due to the fact that the scalar product $(\phi, \phi)_{\mathcal{A}_{\theta}}$ takes its values in the algebra $\mathcal{A}_{\theta}$. The vertex (3.9) always has the $A$-field attached to the right when 
following the arrows of the $\phi$-field. Exchanging $\phi$ and $\phi^{\dagger}$ would involve the scalar product $(\phi, \phi)_{\mathcal{B}_{\theta}}$ with values in the commutant of $\mathcal{A}_{\theta}$ in $\mathscr{L}\left(\mathscr{H}_{\mathcal{E}}\right)$. (Recall that $\mathscr{H}_{\mathcal{E}}$ is the Hilbert space obtained by completion of $\mathscr{E}$ with respect to the norm subordinate to the scalar product $\langle\cdot, \cdot\rangle_{\mathcal{E}}=\operatorname{Tr}_{\mathcal{A}_{\theta}}\left[(\cdot, \cdot)_{\mathcal{A}_{\theta}}\right]$.) We shall come back to this point when discussing duality.

It is worthwhile to notice that all the operators entering in the Feynman rules extend to bounded operators on $\mathscr{H}_{\mathcal{E}}$. Instead being interpreted as a distribution, a typical regularised Feynman diagram with $N$ incoming and $N$ outgoing lines will then be conveniently seen as a linear map from $\mathscr{H}^{\otimes N}$ to itself.

For instance, an arbitrary diagram with $2 \mathrm{~N}$ external legs is obtained as follows:

- Draw $N$ oriented lines representing the propagation of the field $\phi$. Each of these lines represents an operator from $\mathscr{H}_{\mathcal{E}}$ to itself, obtained by multiplying propagators $H^{-1}$ and interactions $U_{\gamma}$.

- If they occur, draw closed oriented loops made of propagators $H^{-1}$ and vertices $U_{\gamma}$ and take the traces of the corresponding operators.

- Relate all interactions by wavy lines representing the propagation of $A$ and sum over all the corresponding $\gamma$.

It is important to know at this stage that the divergences we will encounter may both come from the traces and the sums over $\gamma$ 's. Because of the orientation of the lines there are no non-trivial symmetry factors, as e.g. in QED.

The simplest diagram in the 2-point function $\mathscr{H}_{\mathcal{E}} \rightarrow \mathscr{H}_{\mathcal{E}}$ is

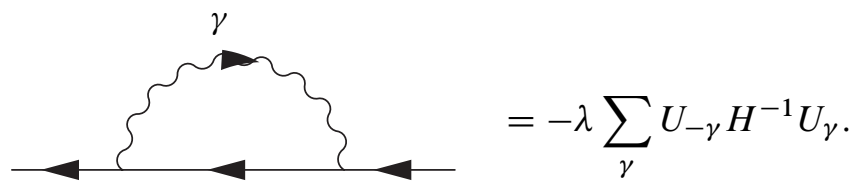

Here we have made a little abuse of notation, using the same symbol $U_{\gamma}$ to denote an element of the noncommutative torus and the operator acting (on the right) on $\mathscr{H}_{\mathcal{E}}$. Also the trace which will appear in the next diagram is not the noncommutative torus trace but the operator trace on $\mathscr{H}_{\mathcal{E}}$. A diagram contributing to the 2-point function involving a closed loop is

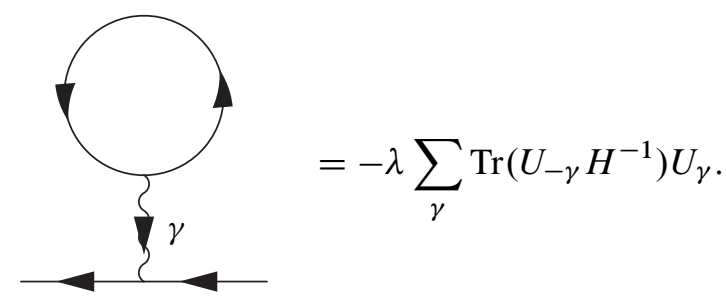


The simplest non-planar diagram contributing to the 2-point function is

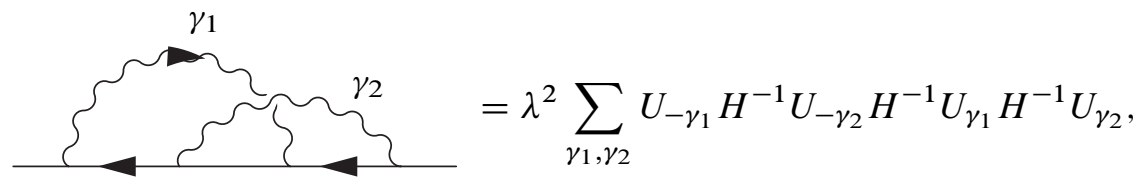

where $\gamma_{2}$ has the same orientation as $\gamma_{1}$. Analogously, let us list a few diagrams contributing to the 4-point function $\mathscr{H}_{\mathcal{E}} \otimes \mathscr{H}_{\mathcal{E}} \rightarrow \mathscr{H}_{\mathcal{E}} \otimes \mathscr{H}_{\mathcal{E}}$, starting with the tree level contribution:

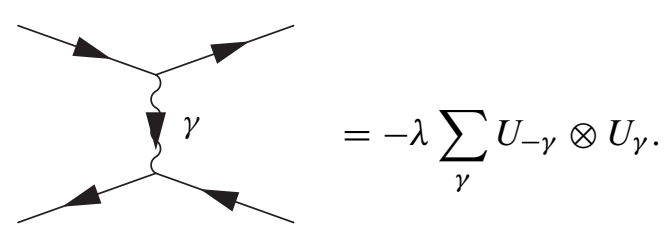

This diagram simply represents the vertex once the $A$-field has been integrated out.

The first non-trivial 4-point diagram with a loop is

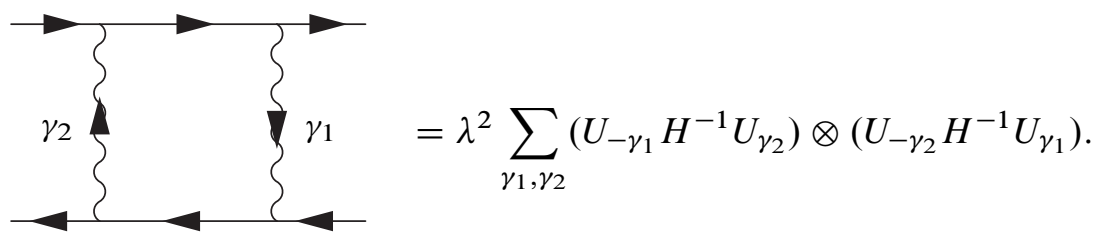

This diagram is planar. A non-planar one can be constructed by exchanging $\gamma_{1}$ and $\gamma_{2}$ in the second tensor product,

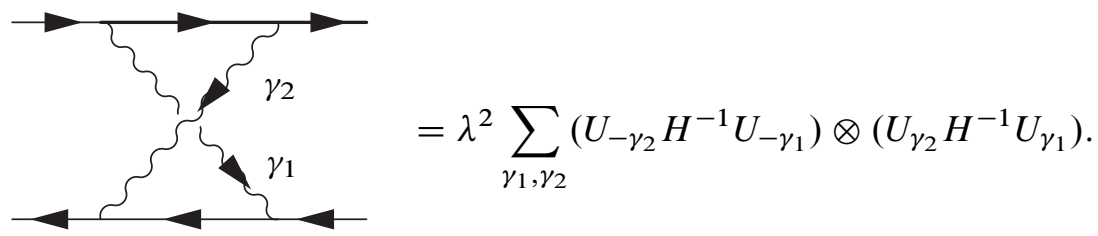

With a trace, the simplest example is

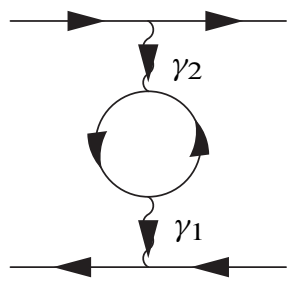

$$
=\lambda^{2} \sum_{\gamma_{1}, \gamma_{2}} \operatorname{Tr}\left(H^{-1} U_{\gamma_{2}} H^{-1} U_{-\gamma_{1}}\right) U_{-\gamma_{2}} \otimes U_{\gamma_{1}}
$$


Finally, let us give a more complicated example contributing to the 6-point function $\mathscr{H}_{\mathcal{E}} \otimes \mathscr{H}_{\mathcal{E}} \otimes \mathscr{H}_{\mathcal{E}} \rightarrow \mathscr{H}_{\mathcal{E}} \otimes \mathscr{H}_{\mathcal{E}} \otimes \mathscr{H}_{\mathcal{E}}$,

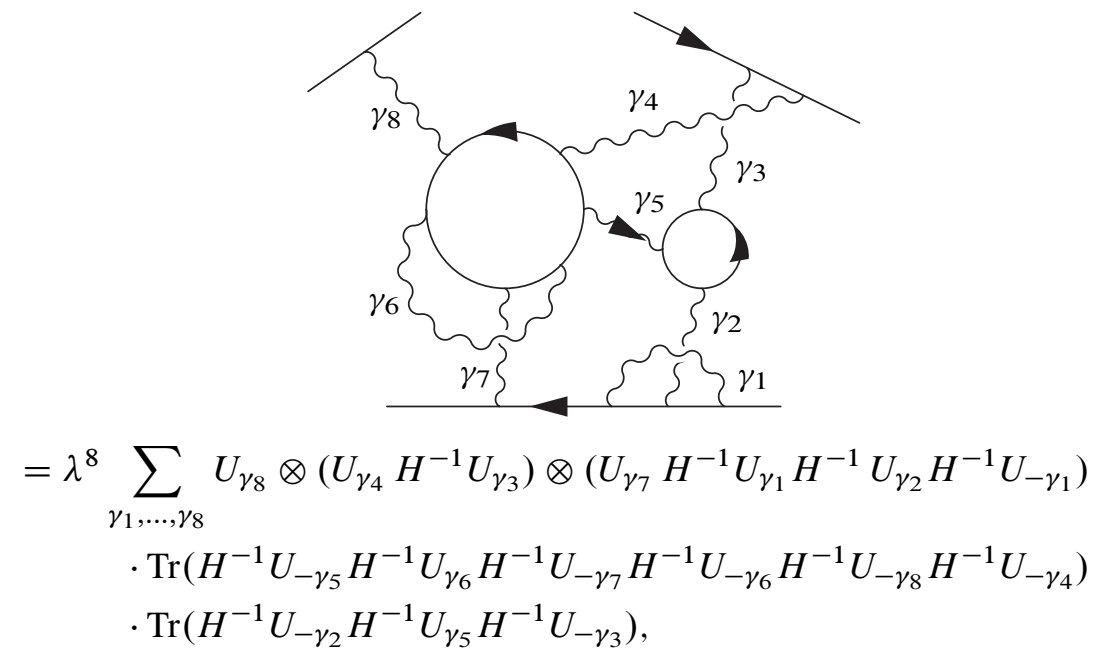

where $\gamma_{2}, \gamma_{3}, \gamma_{4}, \gamma_{7}, \gamma_{8}$ leave the loops and the internal $\gamma_{1}$ and $\gamma_{6}$ first leave and then arrive, according to the orientation.

Let us note that on the definition of an 1PI diagram we impose the irreducibility condition only for the $\phi$-lines, not for the $A$-lines. In fact, only the $\phi$-lines are really internal lines. Thus we reserved the terminology to the latter. The wavy $A$ lines are just a convenient way to visualise the interaction and serve to indicate the identifications of the operators $U_{\gamma}$ inserted in the diagram.

For the simplest example of a Heisenberg module in $x$-space, we have seen that the theory is invariant under the Langmann-Szabo duality, see (2.42). This is a general fact which follows from the existence of two compatible scalar products on the projective module $\mathcal{E}$ under fairly general conditions [25]. Indeed, if we denote by $\mathscr{B}_{\theta}$ the endomorphism algebra $\operatorname{End}_{\mathcal{A}_{\theta}}(\mathcal{E})$, then one defines a scalar product $(\cdot, \cdot)_{\mathcal{B}_{\theta}}$ on $\mathcal{E}$, which is linear with respect to the left action of $\mathscr{B}_{\theta}$ on the first variable and anti-linear in the second one. The two scalar products are compatible in the sense that

$$
\phi(\chi, \psi)_{\mathcal{A}_{\theta}}=(\phi, \chi)_{\mathcal{B}_{\theta}} \psi
$$

for any $\phi, \chi, \psi \in \mathcal{E}$. The algebra $\mathscr{B}_{\theta}$ is in general another noncommutative torus constructed with the dual lattice. If we denote by $\operatorname{Tr}_{\mathcal{B}_{\theta}}$ its normalised trace, then the interaction can be transformed as

$$
\begin{aligned}
\operatorname{Tr}_{\mathcal{A}_{\theta}}\left[\left(\phi_{1}, \phi_{2}\right)_{\mathcal{A}_{\theta}}\left(\phi_{3}, \phi_{4}\right)_{\mathcal{A}_{\theta}}\right] & =\operatorname{Tr}_{\mathcal{A}_{\theta}}\left[\left(\phi_{1}, \phi_{2}\left(\phi_{3}, \phi_{4}\right)_{\mathcal{A}_{\theta}}\right)_{\mathcal{A}_{\theta}}\right] \\
& =\operatorname{Tr}_{\mathcal{A}_{\theta}}\left[\left(\phi_{1},\left(\phi_{2}, \phi_{3}\right)_{\mathcal{B}_{\theta}} \phi_{4}\right)_{\mathcal{A}_{\theta}}\right] \\
& =\operatorname{Tr}_{\mathcal{B}_{\theta}}\left[\left(\left(\phi_{2}, \phi_{3}\right)_{\mathcal{B}_{\theta}} \phi_{4}, \phi_{1}\right)_{\mathcal{B}_{\theta}}\right] \\
& =\operatorname{Tr}_{\mathcal{B}_{\theta}}\left[\left(\phi_{4}, \phi_{1}\right)_{\mathcal{B}_{\theta}}\left(\phi_{2}, \phi_{3}\right)_{\mathcal{B}_{\theta}}\right]
\end{aligned}
$$


where we have used $\operatorname{Tr}_{\mathcal{A}_{\theta}}\left[(\phi, \chi)_{\mathcal{A}_{\theta}}\right]=\operatorname{Tr}_{\mathcal{B}_{\theta}}\left[(\chi, \phi)_{\mathcal{B}_{\theta}}\right]$. We have also labelled explicitly all the fields in order to better visualise the manipulation we have made.

At the level of the Feynman diagrams, especially for the (total) vertex, this duality reads

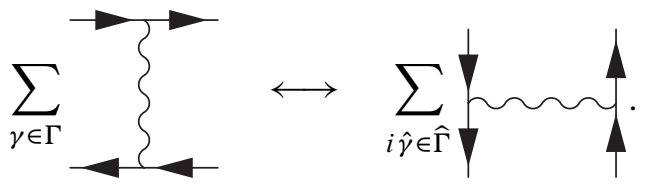

Then it is easy to see that this duality exchanges different types of diagrams: for planar diagrams lines and bubbles are exchanged. The dual of an arbitrary diagram is constructed in two steps. First, replace any wavy line by a double line. Then, in each double line, relate the two lines by a new wavy line. The dual theory can also be written using a Hubbard-Stratonovitch transform with an auxiliary field $B \in \mathcal{B}_{\theta}$, but now the opposite convention has to be used: following the arrows the field $B$ leaves on the left.
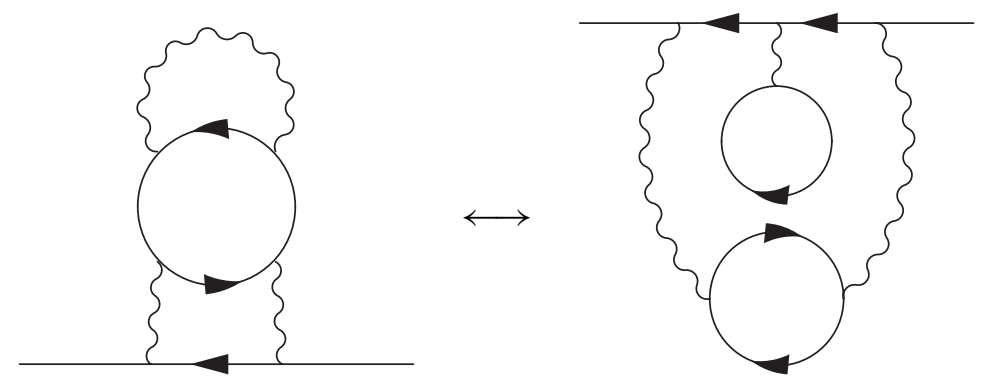

For the simple Heisenberg module obtained from Schwartz functions on $\mathbb{R}$, the scalar product with values in $\mathscr{B}_{\theta}=\mathcal{A}_{1 / \theta}$ is simply

$$
\begin{aligned}
(\chi, \phi)_{\mathcal{A}_{1 / \theta}} & =\frac{1}{\theta} \sum_{\hat{\gamma} \in \hat{\Gamma}}\left\langle\phi, U_{\hat{\gamma}} \chi\right\rangle_{L^{2}(\mathbb{R})} U_{-\hat{\gamma}} \\
& =\frac{1}{\theta} \sum_{\hat{\gamma} \in \hat{\Gamma}}\left(\int_{\mathbb{R}} d x \bar{\phi}(x) e^{\frac{i \pi m n}{\theta}} \chi(x+m) e^{\frac{2 i \pi n x}{\theta}}\right) U_{-\hat{\gamma}} .
\end{aligned}
$$

One passes from one scalar product to the other by Poisson re-summation. In the study of the renormalisability it will prove to be helpful to use the Poisson re-summation for some of the $\gamma$ 's only. This corresponds to a duality operation on only some of the vertices. 
3.2. Reduced theory. To connect with the standard analysis of divergences of a field theory in the Moyal plane [15], it is helpful to write the propagator as

$$
H^{-1}=\int_{0}^{\infty} d \alpha e^{-\alpha H} .
$$

Therefore the contribution of any diagram $G$ with $N$ internal lines and $M$ vertices is expressed as an integral over $N$ Schwinger parameters and a sum over $M$ lattice elements

$$
\int d \alpha_{1} \ldots d \alpha_{N} \sum_{\gamma_{1}, \ldots, \gamma_{M}} I_{G}\left(\alpha_{1}, \ldots, \alpha_{N} ; \gamma_{1}, \ldots, \gamma_{M}\right) .
$$

We refer to the next section for a precise evaluation of this integral in the Bargmann representation.

At a heuristic level it is expected that all the divergences arise from the regions of integration in which one or several Schwinger parameters go to zero. Indeed, if all the Schwinger parameters are different from zero, the traces in $I_{G}$ as well as the sums over lattice elements $\gamma$ are convergent as tempered distributions. We shall see in the next sections the details of this fact for all 1-loop diagrams.

Thus it is interesting to analyse $I_{G}\left(\alpha_{1}, \ldots, \alpha_{N} ; \gamma_{1}, \ldots, \gamma_{M}\right)$ when all Schwinger parameters are set to zero. In this case there are no propagators anymore and only the insertions of $U_{\gamma}$ remain. For any trace around a closed loop of internal lines, there is a divergence proportional to the trace of the product of the operators $U(\gamma)$ along the loop. If we renormalise the trace such that the trace of the identity is 1 , this reduced vertex reads
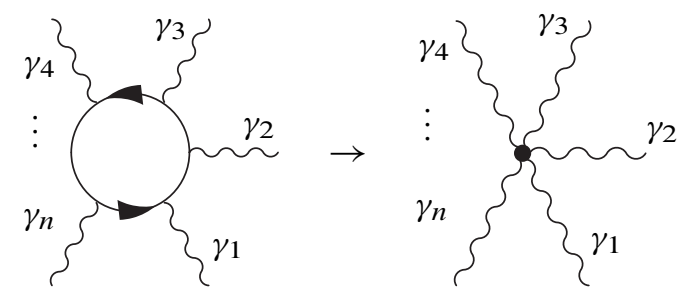

$$
\prod_{i<j} e^{-i \pi \theta\left(\gamma_{i}, \gamma_{j}\right)} \delta\left(\gamma_{1}+\cdots+\gamma_{M}\right),
$$

where the ordering is given by the orientation of the loop. By the same method we can reduce an open line. It is important to keep in mind that the reduced diagrams are also to be thought of as ribbon diagrams because the cyclic orders at the vertices matters:

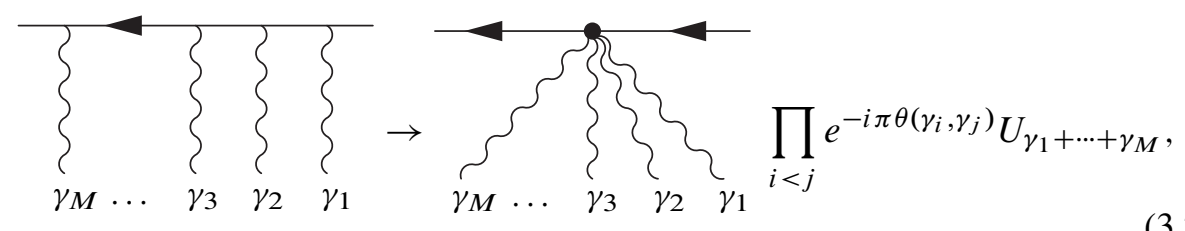


where the ordering is again given by the orientation of the line.

The reduced theory behaves as a very simple model with vertices given by the previous two types of diagrams, propagators equal to one and momenta corresponding to the lattice elements. External legs of the reduced diagrams are given by the external legs of the lines that have been reduced and the sum of the lattice elements along the line corresponds to the external momenta of the reduced diagram. Momentum is conserved at the internal vertices and only the loops of the reduced diagram involve a non-trivial summation. Besides, any diagram carries a phase factor, which is readily computed using the techniques introduced by T. Filk [15].

It follows from this work that the phase associated with a planar diagram (i.e., a diagram that can be drawn without crossings) depends only on the external momenta of the reduced diagram. Therefore the diagram is independent of the loop momenta flowing into the reduced diagram and the corresponding summation is divergent. For example, in the ladder diagram contributing to the 4-point function, the summation reads

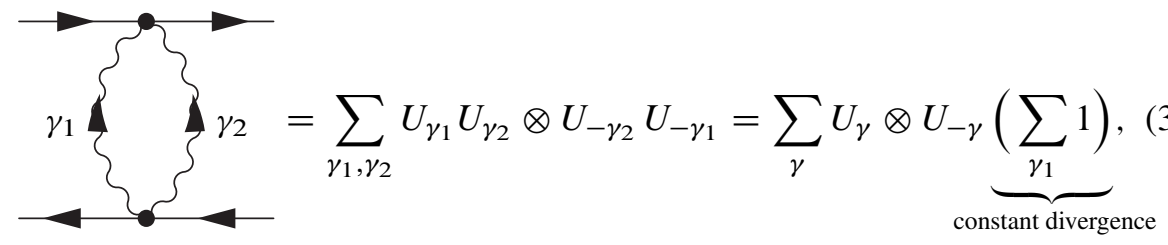

with $\gamma=\gamma_{1}+\gamma_{2}$. The remaining sum over $\gamma_{1}$ is obviously divergent and its divergent part is proportional to the vertex $\sum_{\gamma} U_{\gamma} \otimes U_{-\gamma}$ so that one can infer that it is renormalisable.

The non-planar analogue of the previous diagram behaves very differently. Its contribution to the 4-point function is

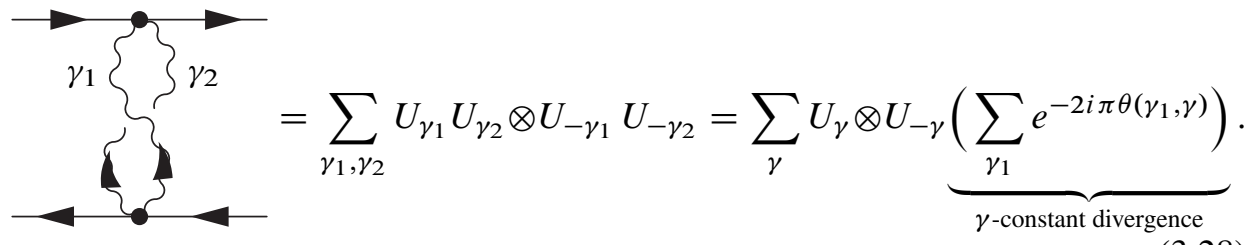

In the integral case the phase factor is trivial and the integral diverges for all $\gamma$ as in the commutative theory. If the phases are all rational, then there is a divergence for those $\gamma$ such that $U_{\gamma}$ is central, for other values of $\gamma$ there is an exact cancellation. In the irrational case there is obviously a divergence when $\gamma=0$. We shall see in the section on 1-loop renormalisation that this is the only divergence when $\theta$ satisfies a Diophantine condition.

All the properties of the noncommutative field theory we have presented in this section are independent of the representation we choose. As such they are best derived 
in a general setting, without using any specific representation for the operators $H$ and $U_{\gamma}$. In the next two sections we shall use the position space (or equivalently, by Langmann-Szabo duality, the momentum space representation) to uncover an analogy with matrix models and with the Grosse-Wulkenhaar model [18], and the holomorphic representation for an explicit evaluation of Feynman diagrams and their divergences.

\subsection{Position space Feynman rules and relations with other models}

3.3.1. Relation with matrix models. Let us come back to the non-local structure of the interaction in $x$-space. For the simplest module, i.e., $\mathcal{E}_{8}=\delta\left(\mathbb{R}^{2}\right)$, the interaction term given by (2.40) can be written as

$$
\int_{\mathbb{R}^{4}} d x d y d z d t V(x, y, z, t) \bar{\phi}(x) \phi(y) \bar{\phi}(z) \phi(t),
$$

with

$$
V(x, y, z, t)=\frac{\lambda}{2} \sum_{m, n \in \mathbb{Z}} \delta(y-x-m \theta) \delta(z-x-m \theta-n) \delta(t-x-n) .
$$

This leads to a vertex

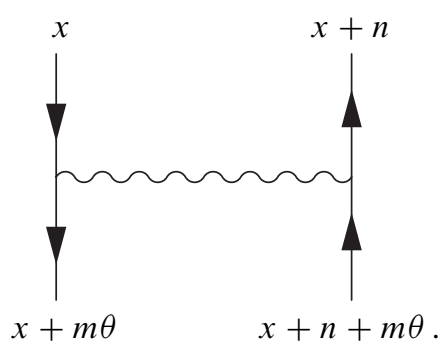

The non-local structure of this interaction given by the vertex (3.30) is intuitively understood as follows. First let us notice that the interaction involves products of the fields at the four points $x, x+m \theta+n, x+n$ and $x+m \theta$. These four points are identical in the quotient space $\mathbb{R} /(\mathbb{Z}+\theta \mathbb{Z})$ so that the theory would be local if it could be formulated on such a quotient. This is possible if $\theta$ is rational, but otherwise the quotient space is badly behaved from a topological viewpoint, namely because $\mathbb{Z}+\theta \mathbb{Z}$ is dense in $\mathbb{R}$ when $\theta$ is irrational. Such a quotient space is related to Kronecker's foliation and is fruitfully understood using the powerful techniques presented in [10]. Here we shall content ourselves with a very rough analysis by disregarding the topological difficulties so that we simply write any real number as $x=[x]+k \theta+l$, where $[x]$ is the class of $x$ in the quotient and $k$ and $l$ are elements 
of $\mathbb{Z}$. Accordingly, the interaction vertex can be written as

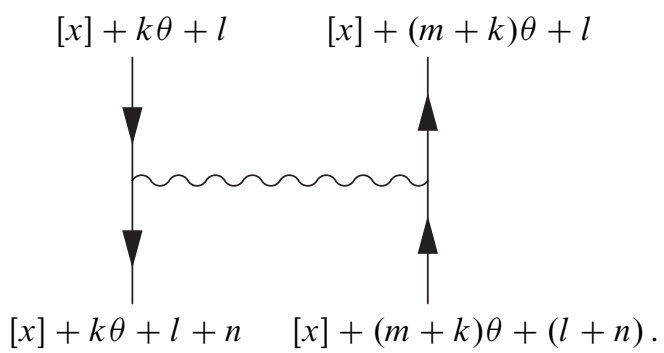

It appears that all the fields are evaluated at a point which corresponds to the same equivalence class $[x]$, whereas the interaction mixes the integers $k$ and $l$ in a particular pattern reminiscent of matrix models. Indeed in a matrix model involving $(p \times q)$-rectangular matrices with an interaction of the type

$$
V\left(M, M^{\dagger}\right)=\frac{\lambda}{2} \operatorname{Tr}\left[M^{\dagger} M M^{\dagger} M\right]=\underset{\substack{0 \leq i_{1}, i_{2} \leq q-1 \\ 0 \leq j_{1}, j_{2} \leq p-1}}{\frac{\lambda}{2}}\left(M^{\dagger}\right)_{i_{1} j_{1}} M_{j_{1} i_{2}}\left(M^{\dagger}\right)_{i_{1} j_{2}} M_{j_{2} i_{1}},
$$

the interaction vertex is

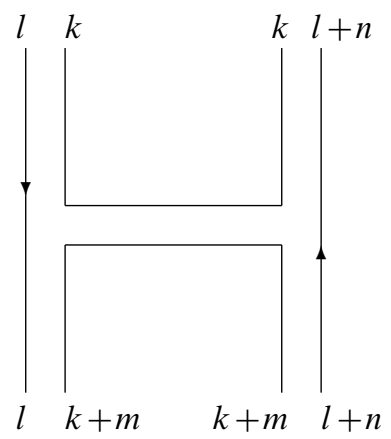

The integers $k, l, k+m$ and $l+n$ correspond to matrix indices $i_{1}, j_{1}, i_{2}$ and $j_{2}$. In the framework of rectangular matrix models the duality (3.19) exchanges $M$ with its adjoint $M^{\dagger}[7]$.

If $\theta=\frac{p}{q}$, with $p$ and $q$ two relatively prime positive integers, then the relation with rectangular matrix models can be established as follows. Define the vector bundle $F \rightarrow \mathbb{T}^{2}$ over a commutative 2 -torus of radius $1 / q$, whose fibres are $(p \times q)$ complex matrices, in terms of its module of smooth sections $\Gamma^{\infty}(F)$. The latter is defined as the set of $(p \times q)$ matrix-valued smooth functions on $[0,1 / q] \times[0,1 / q]$, which are periodic in the second variable but only quasi-periodic in the first:

$$
\begin{aligned}
& M\left(x, y+\frac{1}{q}\right)=M(x, y), \\
& M\left(x+\frac{1}{q}, y\right)=\Omega_{p}^{a}(q y) M(x, y) \Omega_{q}^{-b}(-q y),
\end{aligned}
$$


where $a$ and $b$ are two integers such that $a q+b p=1$ and $\Omega_{N}(y)$ is the $N \times N$ matrix defined by

$$
\Omega_{N}(y)=\left(\begin{array}{cccc}
0 & 1 & & \\
& \ddots & \ddots & \\
& & \ddots & 1 \\
e^{2 i \pi y} & & & 0
\end{array}\right) .
$$

Now given $\phi \in \delta(\mathbb{R})$, one associates an element of $\Gamma^{\infty}(F)$ via the map $\rho$

$$
\begin{aligned}
\rho: \delta(\mathbb{R}) & \rightarrow \Gamma^{\infty}(F), \\
\phi(x) & \mapsto M_{i j}(x, y)=\sum_{n \in \mathbb{Z}} \phi\left(x+\frac{i q+j p+n p q}{q}\right) e^{-2 i \pi n q y},
\end{aligned}
$$

with $i \in\{0,1, \ldots, p-1\}$ and $j \in\{0,1, \ldots, q-1\}$. Using the identity

$$
\left[\Omega_{N}^{n}(y)\right]_{i j}=\sum_{k} \delta_{j, i+n-k N} e^{2 i \pi k y},
$$

where only one term in the sum is non-zero, it is easy to prove that $M$ satisfies the boundary conditions (3.34). Conversely, one can define a function $\phi \in \delta(\mathbb{R})$ from a matrix $M \in \Gamma^{\infty}(F)$ using the map $\rho^{*}$ (the notation will be justified soon):

$$
\rho^{*}: \Gamma^{\infty}(F) \rightarrow \S(\mathbb{R}), \quad M(x, y) \mapsto \phi(x)=q \int_{0}^{\frac{1}{q}} d y M_{00}(x, y) .
$$

From the boundary conditions (3.34), it is straightforward to see that the latter is well defined as an element of $\delta(\mathbb{R})$.

The map $\rho$ establishes an isomorphism between $\delta(\mathbb{R})$ and $\Gamma^{\infty}(F)$, whose inverse is $\rho^{*}$. Moreover, $\rho$ and $\rho^{*}$ extend to unitary operators at the level of $L^{2}$-completions. To this aim, let $L^{2}\left(\mathbb{T}^{2}, F\right)$ be the completion of $\Gamma^{\infty}(F)$ with respect to the norm $\|\cdot\|_{F}$ induced from the scalar product

$$
\langle M, N\rangle_{F}=q \int_{0}^{1 / q} d x \int_{0}^{1 / q} d y \operatorname{Tr}\left[M^{\dagger}(x, y) N(x, y)\right] .
$$

Lemma 3.1. For $\theta=\frac{p}{q}$, with $p$ and $q$ relatively prime positive integers, the map $\rho$ is a unitary operator from $L^{2}(\mathbb{R})$ to $L^{2}\left(\mathbb{T}^{2}, F\right)$, whose adjoint is $\rho^{*}$.

Proof. First observe that the maps (3.36), $\rho: \delta(\mathbb{R}) \rightarrow \Gamma^{\infty}(F)$, and (3.38), 
$\rho^{*}: \Gamma^{\infty}(F) \rightarrow \delta(\mathbb{R})$, are inverse each other. Indeed, for any $\phi \in \delta(\mathbb{R})$, we have

$$
\begin{aligned}
\left(\rho^{*} \rho \phi\right)(x) & =q \int_{0}^{1 / q} d y(\rho \phi)_{00}(x, y) \\
& =q \int_{0}^{1 / q} d y \sum_{n \in \mathbb{Z}} \phi(x+n p) e^{-2 i \pi n q y}=\sum_{n \in \mathbb{Z}} \delta_{n, 0} \phi(x+n p)=\phi(x) .
\end{aligned}
$$

Conversely, for any $M \in \Gamma^{\infty}(F)$ we find

$$
\begin{aligned}
\left(\rho \rho^{*} M\right)_{i j}(x, y) & =\sum_{n \in \mathbb{Z}}\left(\rho^{*} M\right)\left(x+\frac{i q+j p+n p q}{q}\right) e^{-2 i \pi n q y} \\
& =q \sum_{n \in \mathbb{Z}} \int_{0}^{1 / q} d z M_{00}\left(x+\frac{i q+j p+n p q}{q}, z\right) e^{-2 i \pi n q y} .
\end{aligned}
$$

Using the boundary conditions (3.34) and the Poisson re-summation formula (in the sense of tempered distributions), the former expression reads

$q \sum_{n \in \mathbb{Z}} \int_{0}^{1 / q} d z M_{i j}(x, z) e^{-2 i \pi n q(y-z)}=q \sum_{n \in \mathbb{Z}} \int_{n / q}^{(n+1) / q} d z M_{i j}(x, z) \delta(q z-q y)=M_{i j}(x, y)$.

Now for $\phi, \chi \in \delta(\mathbb{R})$, denote by $M, N \in \Gamma^{\infty}(F)$ the corresponding matrices. Let us also introduce the bijective map

$$
\begin{aligned}
\Xi:\{0,1, \ldots, p-1\} \times\{0,1, \ldots, p-1\} \times \mathbb{Z} & \rightarrow \mathbb{Z}, \\
(i, j, n) & \mapsto i q+j p+n p q .
\end{aligned}
$$

Using this map one has

$$
\begin{aligned}
\langle M, N\rangle_{F} & =q \int_{0}^{\frac{1}{q}} d x \int_{0}^{\frac{1}{q}} d y \operatorname{Tr}\left[M^{\dagger}(x, y) N(x, y)\right] \\
& =\sum_{\Xi(i, j, n) \in \mathbb{Z}} \int_{0}^{\frac{1}{q}} d x \bar{\phi}\left(x+\frac{\Xi(i, j, n)}{q}\right) \chi\left(x+\frac{\Xi(i, j, n)}{q}\right) \\
& =\int_{-\infty}^{+\infty} d x \bar{\phi}(x) \chi(x)=\langle\phi, \chi\rangle_{L^{2}(\mathbb{R})} .
\end{aligned}
$$

Therefore, $\|\rho \phi\|_{F}=\|\phi\|_{L^{2}(\mathbb{R})}$ and $\left\|\rho^{*} M\right\|_{L^{2}(\mathbb{R})}=\|M\|_{F}$ for any $\phi \in \delta(\mathbb{R})$ and $M \in \Gamma^{\infty}(F)$. By density, this proves that $\rho$ and $\rho^{*}$ extend to isometries on $L^{2}(\mathbb{R})$ and $L^{2}\left(\mathbb{T}^{2}, F\right)$ and that they are adjoint each other. This concludes the proof since one has already checked that $\rho^{*} \rho=1_{L^{2}(\mathbb{R})}, \rho \rho^{*}=1_{L^{2}\left(\mathbb{T}^{2}, F\right)}$. 
It is worthwhile to notice that the map $\rho$ possesses more structures. In particular, it preserves the module structure, where $\Gamma^{\infty}(F)$ is equipped with a left action of the bundle algebra of $(p \times p)$-matrices over $\mathbb{T}^{2}$, with boundary conditions:

$$
\begin{aligned}
& A\left(x, y+\frac{1}{q}\right)=A(x, y), \\
& A\left(x+\frac{1}{q}, y\right)=\Omega_{p}^{a}(q y) A(x, y) \Omega_{p}^{a}(q y)^{\dagger} .
\end{aligned}
$$

In particular, the two covariant derivatives defining the connection (2.38) on the Schwartz space translate into

$$
\nabla_{1}=\frac{\partial}{\partial y}-\frac{2 i \pi p x}{q}+A \cdot+\cdot B \quad \text { and } \quad \nabla_{2}=\frac{\partial}{\partial x}
$$

on the rectangular matrices. In this definition, $A$ and $B$ are two square $p \times p$ and $q \times q$ constant diagonal matrices acting by left and right multiplication. Their diagonal elements are

$$
A_{k k}=-2 i \pi p k \quad \text { and } \quad B_{l l}=-\frac{2 i \pi}{p} l .
$$

Therefore, at rational $\theta$, the Heisenberg module simply describes a bundle of rectangular matrices over an ordinary torus. These relations generalise the one given in [22], which corresponds to the case $p=q=1$.

Consider now a rectangular matrix model, with matrix-valued functions satisfying the boundary conditions (3.34) and an action given by

$$
\begin{aligned}
S\left[M, M^{\dagger}\right]= & \int_{0}^{\frac{1}{q}} d x \int_{0}^{\frac{1}{q}} d y \operatorname{Tr}\left[\nabla_{\mu} M^{\dagger}(x, y) \nabla^{\mu} M(x, y)\right. \\
& \left.+\mu_{0}^{2} M^{\dagger}(x, y) M(x, y)+\frac{\lambda}{2}\left(M^{\dagger}(x, y) M(x, y)\right)^{2}\right] .
\end{aligned}
$$

If we express the matrix $M$ in terms of $\phi$ via the map $\rho$, the matrix model action agrees with the Heisenberg module action (2.40) up to a factor $q$,

$$
q S\left[M, M^{\dagger}\right]=S[\phi, \bar{\phi}] .
$$

The equality for the quadratic part of the action is already contained in the Lemma 3.1. The interaction term can be treated along the same lines. The matrix model interaction reads

$$
\frac{q \lambda}{2} \sum_{i, j, k, l} \int_{0}^{\frac{1}{q}} d x \int_{0}^{\frac{1}{q}} d y \bar{M}_{i j}(x, y) M_{i k}(x, y) \bar{M}_{l k}(x, y) M_{l j}(x, y) .
$$

Expressing $M$ in terms of $\phi$ and integrating over $y$ yields

$$
\begin{aligned}
\frac{\lambda}{2} \sum_{N, N^{\prime}, N^{\prime \prime}} \sum_{i, j, k, l} \int_{0}^{\frac{1}{q}} d x \bar{\phi}\left(x+\frac{i q+j p+N p q}{q}\right) \phi\left(x+\frac{i q+k p+N^{\prime} p q}{q}\right) \\
\cdot \bar{\phi}\left(x+\frac{l q+k p+\left(N^{\prime}+N^{\prime \prime}-N\right) p q}{q}\right) \phi\left(x+\frac{l q+j p+N^{\prime \prime} p q}{q}\right) .
\end{aligned}
$$


After a shift of the integration variable by $\Xi(i, j, N)$ and a change of summation indices

$$
\begin{aligned}
& N^{\prime}-j \rightarrow N^{\prime}, \quad k-j \rightarrow k \\
& N^{\prime \prime}-i \rightarrow N^{\prime \prime}, \quad l-i \rightarrow l
\end{aligned}
$$

the interaction reads

$$
\begin{aligned}
\frac{\lambda}{2} \sum_{\Xi(i, j, N)} \sum_{N^{\prime}, N^{\prime \prime}} \sum_{k, l} \int_{\Xi(i, j, N)} \frac{\Xi(i, j, N)+1}{q} d x \bar{\phi}(x) \phi\left(x+\frac{k p+\left(N^{\prime}\right) p q}{q}\right) \\
\cdot \bar{\phi}\left(x+\frac{l q+k p+\left(N^{\prime}+N^{\prime \prime}\right) p q}{q}\right) \phi\left(x+\frac{l q+N^{\prime \prime} p q}{q}\right) .
\end{aligned}
$$

Finally, the identification with the vertex in $x$-space

$$
\frac{\lambda}{2} \int_{+\infty}^{-\infty} d x \sum_{m, n \in \mathbb{Z}} \bar{\phi}(x) \phi\left(x+m \frac{p}{q}\right) \bar{\phi}\left(x+m \frac{p}{q}+n\right) \phi(x+n)
$$

follows from the euclidian division of $m$ by $q$ and of $n$ by $p$,

$$
\begin{aligned}
m & =k+q N^{\prime}, \\
n & =l+p N^{\prime \prime} .
\end{aligned}
$$

Let us note that the action in terms of the Schwartz function $\phi$ involves only the ratio $\frac{p}{q}$ and does not depend separately on $p$ and $q$. Therefore, it is suited to the study of the limit case

$$
p \rightarrow \infty \quad \text { and } \quad q \rightarrow \infty \quad \text { with } \frac{p}{q} \rightarrow \theta,
$$

where $\theta$ is a fixed positive real number. Thus, one has

$$
\lim _{\substack{p, q \rightarrow \infty \\ p / q \rightarrow \theta}} \int[D M]\left[D M^{\dagger}\right] e^{-q S\left[M, M^{\dagger}\right]} \mathcal{O}\left(M, M^{\dagger}\right)=\int[D \phi][D \bar{\phi}] e^{-S[\phi, \bar{\phi}]} \mathcal{O}(\phi, \bar{\phi}) .
$$

In this equation one has to keep in mind that the matrix-valued function $M$ satisfies the boundary condition (3.34), which means that its period, along the two directions $x$ and $y$, is $1 / q$, which goes to zero as $q \rightarrow \infty$. In this respect the quantum field theory over the Heisenberg module corresponds to a twisted reduced matrix model, the size of the underlying space going to zero. In the next sections we shall see that the renormalisation properties of these models involve some number theoretical properties of $\theta$ for non-planar diagrams.

3.3.2. Relation with the Grosse-Wulkenhaar model. Recall that in [18], [26] it is proven that the duality-covariant $\phi_{4}^{4}$-theory on the Moyal hyperplane is renormalisable 
to all orders in perturbation theory. In the exact self-dual case $(\Omega=1$ in the language of [18]), the theory is defined by the following action functional

$$
S[\phi]:=\frac{1}{2} \int d^{4} x \phi(x)\left(\triangle+X^{2}+\mu_{0}^{2}\right) \phi(x)+\frac{\lambda}{4} \int d^{4} x(\phi \star \phi \star \phi \star \phi)(x),
$$

where $\Delta=-\partial^{\mu} \partial_{\mu}$ is the Laplacian and $X^{2}=X^{\mu} X_{\mu}$ with $X^{\mu}=2\left(\theta^{-1}\right)^{\mu v} x_{v}$. Here $\theta_{\mu \nu}=-\theta_{\nu \mu} \in \mathbb{R}$ are the components of the deformation matrix which defines the Moyal product $\star$, and $x^{\mu}$ denotes the ordinary Cartesian coordinate on $\mathbb{R}^{4}$.

It is worthwhile to notice that the kinetic term of that theory is exactly the same as in the model we study here: it is given by an harmonic oscillator Hamiltonian. But the origin of this term is quite different. For the action (3.53), the term $X^{2}$ has been "added by hand" to cure the UV/IR-mixing problem. More specifically, such a term is required by the renormalisation flow. But to see it one has to formulate the theory in the matrix base (see [18]). Moreover the presence of such term allows to restore the Langmann-Szabo duality for the entire action. For the Heisenberg module, the harmonic oscillator potential has a clear geometric origin since it comes from the connection (2.38). In particular, whereas the frequency of the oscillator is a free parameter in [26] (and has to be renormalised for $\Omega<1$ ), this is not the case in our model: we will see in the next section that the value of this parameter is preserved by the renormalisation flow. However, in spite of the presence of the confining potential, the $\phi_{4}^{4}$-theory on the Heisenberg module will still suffer from an UV/IR-mixing. Fortunately, in this case the UV/IR-entanglement is easy to cure (at least at 1-loop) by the adjunction of another interaction term.

To investigate the analogies and differences of the interactions of the two models, it is useful to rewrite the vertex (3.30) as

$$
V(x, y, z, t)=\frac{\lambda}{2^{2 d} \theta^{2 d}} \sum_{m, n \in \mathbb{Z}^{\mathbb{d}}} e^{\frac{2 i \pi}{\theta} m(x-y)} \delta(x-y+z-t) e^{2 i \pi n(x-t)} .
$$

This formula can be obtained from (3.30) after a Poisson re-summation on the index $m$. Here we have considered the module $\delta\left(\mathbb{R}^{d}\right)$ over a $2 d$-dimensional noncommutative torus, with deformation matrix of the form (2.68). On the other hand, the interaction vertex associated to (3.53) in $d$-dimensions is given by

$$
V_{\star}(x, y, z, t)=\frac{\lambda}{\pi^{d} \theta^{d}} e^{2 i \theta^{-1}(x, y)} \delta(x-y+z-t) e^{2 i \theta^{-1}(z, t)},
$$

where $\theta^{-1}$ is the antisymmetric bilinear form associated to the inverse deformation matrix $\theta_{\mu \nu}$. This leads to a correspondence between the field theory on the Heisenberg module in dimension $2 d$ (the dimension of the underlying noncommutative torus) and the field theory on the Moyal plane of dimension $d$. This correspondence relates the 
phases in the interactions as

$$
e^{2 i \theta^{-1}(x, y)} \leftrightarrow \sum_{m \in \mathbb{Z}^{d}} e^{\frac{2 i \pi m}{\theta}(y-x)} \text { and } e^{2 i \theta^{-1}(z, t)} \leftrightarrow \sum_{n \in \mathbb{Z}^{d}} e^{2 i \pi n(x-t)} .
$$

The dissymmetry between the two phases has a deep impact on the power counting of the two theories: the correct analogue of the commutative $\phi_{4}^{4}$-theory on the noncommutative hyperplane is made out of the Moyal product on $\mathbb{R}^{4}$, whereas in the non-trivial module case, it has to be formulated on $\delta\left(\mathbb{R}^{2}\right)$, not on $\delta\left(\mathbb{R}^{4}\right)$. Indeed, it is the dimension of the noncommutative torus, not of the representation space that matters. This leads to the following question: how can it be that two theories with the same propagator in different dimensions behave the same? The answer comes precisely from dissymmetry between the vertices (3.54) and (3.55).

To see how the vertex affects the evaluation of the Feynman diagrams, let us have a look at the 1-loop planar 2-point function associated to a quartic interaction for the two models. In the case of the projective module over a $(2 d)$-noncommutative torus, if we evaluate the diagram (3.11) in external fields $\varphi_{1}, \varphi_{2} \in \delta\left(\mathbb{R}^{d}\right)$, we obtain up to a numerical factor

$$
\operatorname{Tr}\left(\left(\varphi_{1}, \varphi_{2}\right)_{\mathcal{A}_{\theta}} H^{-1}\right)=\sum_{\gamma \in \Gamma} \operatorname{Tr}\left(U_{-\gamma} H^{-1}\right)\left\langle\varphi_{1}, \varphi_{2} U_{\gamma}\right\rangle_{L^{2}\left(\mathbb{R}^{d}\right)} .
$$

Recall that in $d$-dimensions the spectrum of $H^{-1}=\left(\Delta+X^{2}\right)^{-1}$ is $(d / 2+|n|)^{-1}$, $n \in \mathbb{N}^{d}$. For $\gamma=0$ (we will see that the terms with $\gamma \neq 0$ give rise to finite contributions) the divergence is logarithmic for $d=1$, quadratic for $d=2$ and so on. The behaviour of this diagram for $d=2$ is reminiscent of a just renormalisable $\phi_{4}^{4}$-theory.

Alternatively, one can study the UV-divergences using a parametric representation (3.23) for the propagator and looking at the small- $\beta$ behaviour. With the help of the Mehler formula, one can express the kernel of $H^{-1}$ as

$$
H^{-1}(x, y)=\left(\frac{\omega}{2 \pi}\right)^{\frac{d}{2}} \int_{0}^{\infty} d \beta \frac{1}{\sinh ^{d / 2}(2 \omega \beta)} e^{-\frac{\omega}{4}\left(\operatorname{coth}(\omega \beta)|x-y|^{2}+\tanh (\omega \beta)|x+y|^{2}\right)},
$$

where $\omega$ is the frequency of the oscillator. If we regularise the theory by setting

$$
H^{-1} \rightarrow H_{\epsilon}^{-1}=\int_{\epsilon}^{\infty} d \beta e^{-\beta H}
$$

then one gets

$$
\operatorname{Tr}\left(H_{\epsilon}^{-1}\right)=\int_{\mathbb{R}^{d}} d x H_{\epsilon}^{-1}(x, x)=\int_{\epsilon}^{\infty} \frac{d \beta}{\sinh ^{d}(\omega \beta)}
$$


This yields the same conclusion: the divergence (in $\varepsilon^{-1 / 2}$ ) is logarithmic for $d=1$, quadratic for $d=2$.

Now the same diagram for the duality-covariant model on the Moyal plane reads

$$
\operatorname{Tr}\left(L\left(\varphi_{1} \star \varphi_{2}\right) H^{-1}\right),
$$

where $L(\varphi)$ denotes the operator of left Moyal multiplication by $\varphi$ (a look at [16] can help the unfamiliar reader to derive such formula). It is then straightforward to verify that the leading divergence of (3.61) is given by

$$
\int_{\mathbb{R}^{d}} d x \varphi_{1} \star \varphi_{2}(x) H_{\epsilon}^{-1}(x, x)=\int_{\mathbb{R}^{d}} d x \varphi_{1} \star \varphi_{2}(x) \int_{\epsilon}^{\infty} d \beta \frac{1}{\sinh ^{d / 2}(2 \omega \beta)} e^{-\omega \tanh (\omega \beta)|x|^{2}} .
$$

Due to the presence of the fields $\varphi_{1}, \varphi_{2}$ inside the $x$-integral, the former can be estimated by

$$
\int_{\mathbb{R}^{d}} d x\left|\varphi_{1} \star \varphi_{2}\right|(x) \int_{\epsilon}^{\infty} d \beta \frac{1}{\sinh ^{d / 2}(2 \omega \beta)} .
$$

Since in the Moyal plane case only even dimensions are allowed (to respect the symplectic pairs structure of the Moyal product), the divergence of the planar 2-point diagram (3.61) is (only) logarithmic for $d=2$ and (as expected) quadratic for $d=4$.

This is a strong evidence that our field theory on $\delta\left(\mathbb{R}^{d}\right)$ does not behave according to the dimension $d$ of the representation space, but according to the dimension $2 d$ of the underlying noncommutative torus!

3.4. Feynman diagrams in the holomorphic representation. From now on we shall work in the holomorphic representation for a Bargmann module in two dimensions obtained by tensoring twice the module of the previous type, i.e., corresponding to a four-dimensional deformation matrix of the form (2.68). In the explicit computation of Feynman diagrams we have to evaluate traces and kernels of products of operators of the type $H^{-1}$ and $U_{\gamma}$ in the holomorphic representation. To this aim, recall that an operator $A$ is said to be an operator with distribution kernel $A\left(z, \bar{z}^{\prime}\right)$ if its action on $\phi \in \mathscr{H}_{B}$ can be written as

$$
A \phi(z)=\int_{\mathbb{C}^{2}} d \mu\left(z^{\prime}, \bar{z}^{\prime}\right) A\left(z, \bar{z}^{\prime}\right) \phi\left(z^{\prime}\right) .
$$

For instance, the identity is an operator with kernel $I\left(z, \bar{z}^{\prime}\right)=e^{\omega \bar{z}^{\prime} z}$, the reproducing kernel of the Bargmann space. By convention, our kernels are holomorphic in the in first variable (associated to an incoming field on the diagram) and anti-holomorphic in the second one (associated to an outgoing field).

Now and for all we will fix a regularisation scheme. To this aim, we define the regularised propagator $H_{\epsilon}^{-1}$ as follows

$$
H_{\epsilon}^{-1}:=\int_{\epsilon}^{\infty} d \beta e^{-\beta H}=H^{-1} e^{-\epsilon H} .
$$


As soon as $\epsilon>0$, the operator $H_{\epsilon}^{-1}$ is a strictly positive compact operator with fast decreasing eigenvalues and in particular trace-class. This operator admits a kernel given by

$$
H_{\epsilon}^{-1}\left(z_{2}, \bar{z}_{1}\right)=\frac{1}{2} \int_{\epsilon}^{\infty} d \beta e^{-\frac{\beta m^{2}}{2}} I\left(z_{2}, e^{-\beta \omega} \bar{z}_{1}\right) .
$$

As such it provides a natural regularisation of the Feynman integrals. Indeed, if we come back to $\mathcal{E}_{8}\left(\mathbb{R}^{2}\right)$ by the inverse Bargmann transform, it is easy to see that $H_{\epsilon}^{-1}(x, y)$ is a function of Schwartz class in both variables (have a look at (3.58) versus (3.59)). Since the vertex (3.30) is a tempered distribution, this means that all the contractions with the regularised propagator are now well defined. Using the Bargmann transform back, it is immediate to see that the same holds for the module $\varepsilon_{B}\left(\mathbb{C}^{2}\right)$.

The regularised propagator, given by the expression (3.66), is graphically represented by a single oriented line

$$
\stackrel{z_{1} \longrightarrow \bar{z}_{2}}{\longrightarrow}=H_{\varepsilon}^{-1}\left(z_{1}, \bar{z}_{2}\right) .
$$

Using the definition (2.51), it is easy to see that the operator $U_{\gamma}$ also admits a kernel given by

$$
U_{\gamma}\left(z_{1}, \bar{z}_{2}\right)=e^{-\frac{\omega}{2}|\gamma|^{2}-\omega \bar{\gamma} z_{1}} I\left(z_{1}+\gamma, \bar{z}_{2}\right)=e^{-\frac{\omega}{2}|\gamma|^{2}+\omega \bar{z}_{2} \gamma} I\left(z_{1}, \bar{z}_{2}-\bar{\gamma}\right) .
$$

Thus in the holomorphic representation the (total or quadri-valent) vertex reads

$$
\begin{aligned}
V\left(z_{1}, \bar{z}_{2}, z_{3}, \bar{z}_{4}\right) & =\frac{\lambda}{2} \sum_{\gamma} U_{\gamma}\left(z_{1}, \bar{z}_{2}\right) U_{-\gamma}\left(z_{3}, \bar{z}_{4}\right) \\
& =\frac{\lambda}{2} \sum_{\gamma} e^{-\omega|\gamma|^{2}} e^{\omega\left(\bar{z}_{2}-\bar{z}_{4}\right) \gamma} I\left(z_{1}, \bar{z}_{2}-\bar{\gamma}\right) I\left(z_{3}, \bar{z}_{4}+\bar{\gamma}\right) \\
& =\frac{\lambda}{2} \sum_{\gamma} e^{-\omega|\gamma|^{2}} I\left(\gamma, \bar{z}_{2}-\bar{z}_{4}\right) I\left(z_{1}, \bar{z}_{2}-\bar{\gamma}\right) I\left(z_{3}, \bar{z}_{4}+\bar{\gamma}\right)
\end{aligned}
$$

and is represented by

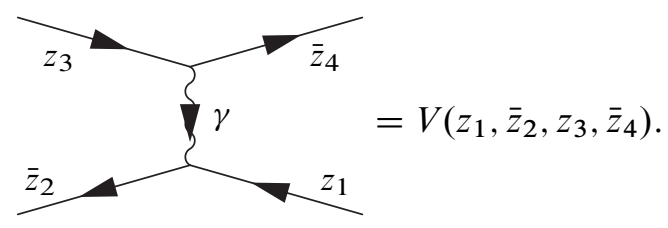

When drawing the Feynman graphs it is important to notice that propagator and vertex are oriented. When following the scalar field arrow, the wavy $\gamma$-line always 
leaves the vertex to the right. Alternatively, to stress the link with the HubbardStratonovich transformation, we may split the vertex (3.70) into two tri-valent vertices

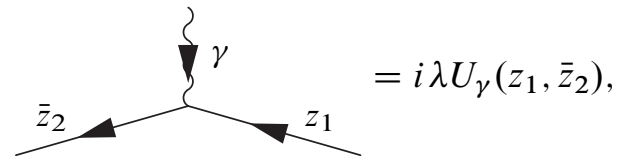

and a propagator for the auxiliary field

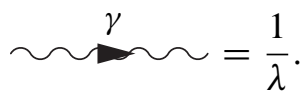

3.5. Parametric representation. A careful analysis of the involved terms shows that a general (regularised) graph always has the following structure:

$$
\begin{aligned}
& \Gamma_{n}\left[z_{1}, \bar{z}_{2}, \ldots, z_{2 N-1}, \bar{z}_{2 N}\right] \\
& \quad=\sum_{\gamma_{1}, \ldots, \gamma_{V}} \int_{\epsilon}^{\infty} \prod_{k=1}^{L}\left(d \beta_{k} e^{-\frac{\mu_{0}^{2}}{2} \beta_{k}}\right) \frac{1}{U_{\Gamma}(\beta)} e^{-\gamma_{i} Q_{i j}(\beta) \bar{\gamma}_{j}+B_{i}(z, \beta) \bar{\gamma}_{i}+\gamma_{i} \overline{\mathcal{B}}_{i}(\bar{z}, \beta),}
\end{aligned}
$$

up to a symmetry factor due to its multiple appearance and a term purely depending on $z_{1}, \ldots, \bar{z}_{2 N}$. The notation means that having chosen an arbitrary graph, $L$ indicates the number of internal scalar field line integrations and $V$ is the number of $\gamma$-lines. Missing indices for $\beta$ or $z$ is just short for taking all. We now give a complete set of rules to construct the matrix $\mathcal{Q}_{i j}(\beta)$ and the linear terms $\mathscr{B}_{i}(z, \beta), \overline{\mathcal{B}}_{i}(\bar{z}, \beta)$ respectively. These can be used to directly write down the result, skipping Gaußian integrations or tracing out loops. For the parametric representation of the $\phi_{4}^{4}$-Moyal model, see [21].

Draw a graph and properly orient the scalar lines as well as the $\gamma$-lines. For each internal line, there is a $\beta$-integration accompanied by a factor $\exp \left(-\frac{\mu_{0}^{2}}{2} \beta\right)$. For each $\gamma$ there is a sum. To construct the diagonal $\mathcal{Q}_{i i}$, one has to take care of five different types of $\gamma$-lines:

(1) Each $\gamma_{i}$ which connects two different external lines gives $\mathbb{Q}_{i i}=\omega$.

(2) A $\gamma_{i}$ connecting a line with a bubble leads to

$$
\mathcal{Q}_{i i}=\frac{\omega}{\left(1-e^{\left.-\omega \sum \beta_{k}\right)}\right.},
$$

where the sum counts the internal integrations of the associated bubble.

(3) Each $\gamma_{i}$ that connects two loops $L_{1}$ and $L_{2}$ generates a term

$$
\mathcal{Q}_{i i}=\omega \frac{\left(1-e^{-\omega \sum \beta_{k}}\right)}{\left(1-e^{-\omega \sum_{L_{1}} \beta_{k}}\right)\left(1-e^{-\omega \sum_{L_{2}} \beta_{k}}\right)},
$$


where $\sum_{L_{1}}$, respectively $\sum_{L_{2}}$, counts the internal integrations of the associated loop and the $\beta$-sum in the numerator counts them all.

(4) An internal $\gamma_{i}$ on an external line gives

$$
\mathcal{Q}_{i i}=\omega\left(1-e^{-\omega \sum \beta_{k}}\right),
$$

where the sum counts the $\beta$-integrations appearing on the part of the external line which is enclosed by that $\gamma_{i}$.

(5) Every $\gamma_{i}$, which represents an internal line inside a bubble generates

$$
\mathcal{Q}_{i i}=\omega \frac{\left(1-e^{-\omega \sum_{H 1} \beta_{k}}\right)\left(1-e^{-\omega \sum_{H 2} \beta_{k}}\right)}{\left(1-e^{-\omega \sum \beta_{k}}\right)},
$$

where the sum in the denominator counts all $\beta$-integrations of the bubble and $\sum_{H_{1}}$ (respectively $\sum_{H_{2}}$ ) counts the $\beta$-integrations of the first (respectively second) half of the bubble, each defined by the points the gamma is attached to.

For the off diagonal elements $\mathcal{Q}_{i j}$ there are essentially two different cases to take care of.

(1) Lines. For a $\gamma_{i}$ which is attached to a chosen line there are three possibilities. The $\gamma_{i}$ may leave or arrive, or it may be 'internal'. Following the orientation of the line, denote by $\gamma_{i}^{(1)}$ the first position the $\gamma_{i}$ is attached to and by $\gamma_{i}^{(2)}$ the (possible) second position. Of course, the latter might be empty. Further, define $\operatorname{sign}\left(\gamma_{i}^{(a)}\right)$ to be +1 if it is arriving and -1 otherwise. The same convention holds for a $\gamma_{j}$ with $i \neq j$. As a last ingredient, define $L_{i j}^{(a, b)}$ to be the sum over internal integration variables $\beta$ enclosed by the positions $\gamma_{i}^{(a)}$ and $\gamma_{j}^{(b)}$ on the line. Then the final contribution is

$$
\mathcal{Q}_{i j}=\omega \sum_{a, b, a<b} \operatorname{sign}\left(\gamma_{i}^{(a)}\right) \operatorname{sign}\left(\gamma_{j}^{(b)}\right) e^{-\omega L_{i j}^{(a, b)}} .
$$

With slight abuse of notation, the ordering $a<b$ is given by the orientation of the line.

(2) Loops. If there is a $\gamma_{i}$ and a $\gamma_{j}$ attached to a chosen loop, the same conventions as in (1) can be used. Here, $L_{i j}^{(a, b)}$ is defined to be the sum over internal integration variables on the shortest loop segment connecting $\gamma_{i}^{(a)}$ and $\gamma_{j}^{(b)}$ following the orientation of the loop. One finds

$$
Q_{i j}=\frac{\omega}{\left(1-e^{\left.-\omega \sum \beta_{k}\right)}\right.} \sum_{a, b} \operatorname{sign}\left(\gamma_{i}^{(a)}\right) \operatorname{sign}\left(\gamma_{j}^{(b)}\right) e^{-\omega L_{i j}^{(a, b)}} .
$$

In this case note that there is no ordering in the sum over $a, b$, but again the convention is to take only $a=2$ (respectively $b=2$ ) if the associated $\gamma$ really is internal. 
The general procedure to construct the matrix $\mathcal{Q}_{i j}$ is then to regard line by line and loop by loop all $\gamma$ 's that are attached to it and to apply the rules given above. For the linear terms $\mathscr{B}_{i}(z, \beta)$ and $\overline{\mathcal{B}}_{j}(\bar{z}, \beta)$ it is sufficient to restrict oneself to a line by line analysis.

(1) For the first one, for every line decorated with an incoming $z_{k}$ and outgoing $\bar{z}_{l}$ denote by $\gamma_{i}^{(1)}$ and $\gamma_{i}^{(2)}$, as before, the positions a $\gamma_{i}$ might be attached to. Define $L_{i}^{(a)}$ to be the sum of internal integration variables on the line segment between $z_{k}$ and $\gamma_{i}^{(a)}$ following the orientation. Note that $L_{i}^{(a)}$ can be the empty sum. One has

$$
\mathscr{B}_{i}=-\omega z_{k} \sum_{a} \operatorname{sign}\left(\gamma_{i}^{(a)}\right) e^{-\omega L_{i}^{(a)}},
$$

and the sum only runs from $a=1$ to $a=2$ if there is a second attachment point.

(2) The second term is constructed completely analogously. It is

$$
\overline{\mathcal{B}}_{i}=\omega \bar{z}_{l} \sum_{a} \operatorname{sign}\left(\gamma_{i}^{(a)}\right) e^{-\omega L_{i}^{(a)}}
$$

with $L_{i}^{(a)}$ being the sum of $\beta$ 's on the oriented line segment between $\gamma_{i}^{(a)}$ and $\bar{z}_{l}$. The pre-factor $U_{\Gamma}(\beta)$ is a product of terms of the form $\left(1-e^{-\omega \sum \beta_{k}}\right)^{d / 2}$. Namely, for each scalar loop $L$ it associates such a factor, that is, for $d=4$ dimensions

$$
U_{\Gamma}(\beta)=\prod_{L}\left(1-e^{-\omega \sum_{L} \beta_{k}}\right)^{2} .
$$

The sum $\sum_{L} \beta_{k}$ stands again for the sum over the internal integration variables defined on the corresponding loop.

As an example consider the first 4-point graph $\Gamma_{4 a}^{(1)}\left[z_{1}, \bar{z}_{2}, z_{3}, \bar{z}_{4}\right]$ (given in (4.13)). For $\mathcal{Q}_{i j}$ and $\mathcal{B}_{i}, \overline{\mathcal{B}}_{i}$ one finds

$$
\mathcal{Q}_{i j}=\frac{\omega}{\left(1-e^{-\omega\left(\beta_{1}+\beta_{2}\right)}\right)}\left(\begin{array}{cc}
1 & -e^{-\omega \beta_{2}} \\
-e^{-\omega \beta_{1}} & 1
\end{array}\right)
$$

and

$$
\mathscr{B}_{i}=\omega\left(\begin{array}{r}
-z_{1} \\
z_{3}
\end{array}\right), \quad \overline{\mathcal{B}}_{i}=\omega\left(\begin{array}{r}
\bar{z}_{2} \\
-\bar{z}_{4}
\end{array}\right)
$$

as well as

$$
U_{\Gamma_{4 a}^{(1)}}=\left(1-e^{-\omega\left(\beta_{1}+\beta_{2}\right)}\right)^{2} .
$$

To complete the construction, finally include for each external line decorated with incoming $z_{k}$ and outgoing $\bar{z}_{l}$ a term $\exp \left\{z_{k} \bar{z}_{l} e^{-\omega \sum_{i} \beta_{i}}\right\}$ with the sum given by the (possibly empty) sum over all integrations variables on that line. 


\section{One-loop renormalisation}

We start this section by showing that for a $\phi^{4}$-theory on the simplest Heisenberg module $\varepsilon_{B}\left(\mathbb{C}^{2}\right)$ over $\mathbb{T}_{\theta}^{4}$, only the 2- and 4-point functions are divergent in the 1-loop approximation.

Proposition 4.1. At one loop, for $N>2$, the $2 N$-point Green's functions are finite as tempered distributions.

Proof. In the 1-loop approximation, the 1PI quantum effective action in external fields $\varphi_{1} \in \mathcal{E}, \bar{\varphi}_{2} \in \mathcal{E}^{*}$ is given by

$$
\Gamma^{(1)}\left[\varphi_{1}, \bar{\varphi}_{2}\right]=\frac{1}{2} \ln \operatorname{det}\left(H^{-1}\left(H+\lambda B_{\varphi_{1}, \varphi_{2}}\right)\right),
$$

where $H$ is the harmonic oscillator Hamiltonian in two dimensions, $\lambda$ is the coupling constant and $B_{\varphi_{1}, \bar{\varphi}_{2}}$ is a bounded operator on $\mathscr{H}_{\mathcal{E}}$ depending only on $\varphi_{1} \in \mathcal{E}, \bar{\varphi}_{2} \in \mathcal{E}^{*}$. ( $\mathcal{E}$ can be either $\mathcal{E}_{8}\left(\mathbb{R}^{2}\right)$ or $\mathcal{E}_{\mathcal{B}}\left(\mathbb{C}^{2}\right)$.) Here the definition of $\Gamma^{(1)}\left[\varphi_{1}, \bar{\varphi}_{2}\right]$ includes the normalisation of the partition function, which yields the $H^{-1}$ pre-factor in the determinant. To see that only the 2- and 4-point functions need regularisation, we perform a Dyson expansion. It formally reads

$$
\begin{aligned}
\left.\ln \operatorname{det}\left(H^{-1}\left(H+\lambda B_{\varphi_{1}, \bar{\varphi}_{2}}\right)\right)\right) & \left.=\operatorname{Tr} \ln \left(1+\lambda H^{-1} B_{\varphi_{1}, \bar{\varphi}_{2}}\right)\right) \\
& \left.=\sum_{n=1}^{\infty}(-)^{n+1} \frac{\lambda^{n}}{n} \operatorname{Tr}\left(\left(H^{-1} B_{\varphi_{1}, \bar{\varphi}_{2}}\right)\right)^{n}\right) .
\end{aligned}
$$

But in $d=2$ dimensions the spectrum of $H^{-1}$ is $\left\{\left(1+n_{1}+n_{2}\right)^{-1}, n_{1}, n_{2} \in \mathbb{N}\right\}$, so that $H^{-1}$ belongs to $\mathscr{L}^{p}\left(\mathscr{H}_{\varepsilon}\right)$ (the $p$-th Schatten class) for $p>2$. Thus only the two first terms of (4.2) are divergent. Moreover, the truncated series (starting at $n=3$ ) is absolutely convergent for reasonable small values of (the absolute value of) the coupling constant:

$$
\begin{aligned}
\left.\mid \sum_{n=3}^{\infty}(-)^{n+1} \frac{\lambda^{n}}{n} \operatorname{Tr}\left(\left(H^{-1} B_{\varphi_{1}, \bar{\varphi}_{2}}\right)\right)^{n}\right) \mid & \leq \sum_{n=3}^{\infty}\left\|B_{\varphi_{1}, \bar{\varphi}_{2}}\right\|^{n} \frac{|\lambda|^{n}}{n}\left\|H^{-1}\right\|^{n-3}\left\|H^{-3}\right\|_{1} \\
& \leq C \sum_{n=3}^{\infty}\left\|B_{\varphi_{1}, \bar{\varphi}_{2}}\right\|^{n} \frac{|\lambda|^{n}}{n}
\end{aligned}
$$


4.1. 2-point sector. At one loop we have the following contributions for the 2-point function:

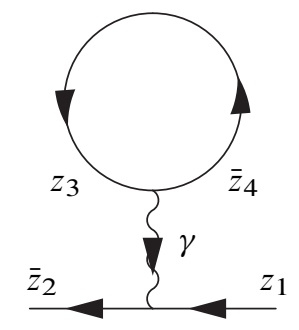

$$
\begin{aligned}
& \Gamma_{2 a}^{(1)}\left[z_{1}, \bar{z}_{2}\right] \\
& \quad=2 \int d \mu\left(z_{3}, \bar{z}_{3}\right) d \mu\left(z_{4}, \bar{z}_{4}\right) V\left(z_{1}, \bar{z}_{2}, z_{3}, \bar{z}_{4}\right) H_{\varepsilon}^{-1}\left(z_{4}, \bar{z}_{3}\right) .
\end{aligned}
$$

The graph of this type appears twice, once with loop connecting $\bar{z}_{4} \rightarrow z_{3}$ (which is shown) and once with loop connecting $\bar{z}_{2} \rightarrow z_{1}$. Replacing $\gamma \mapsto-\gamma$ shows that both contributions are identical.

The $z_{4}$-integration is directly eliminated using one reproducing kernel of the vertex (3.70), and we are left with

$$
\begin{aligned}
\Gamma_{2 a}^{(1)}\left[z_{1}, \bar{z}_{2}\right]=\frac{\lambda}{2} I\left(z_{1}, \bar{z}_{2}\right) \int_{\varepsilon}^{\infty} d \beta \sum_{\gamma} \int d \mu(z, \bar{z}) e^{-\frac{\beta \mu_{0}^{2}}{2}} \\
\cdot e^{-\omega\left(|\gamma|^{2}-|z|^{2} e^{-\beta \omega}+\bar{z} \gamma e^{-\beta \omega}-\bar{\gamma} z+\bar{\gamma} z_{1}-\bar{z}_{2} \gamma\right)} .
\end{aligned}
$$

Now the Gaußian $z$-integral can be evaluated to give

$$
\Gamma_{2 a}^{(1)}\left[z_{1}, \bar{z}_{2}\right]=\frac{\lambda}{2} I\left(z_{1}, \bar{z}_{2}\right) \int_{\varepsilon}^{\infty} d \beta \sum_{\gamma} \frac{e^{-\frac{\beta \mu_{0}^{2}}{2}} e^{-\omega\left(\frac{|\gamma|^{2}}{1-e^{-\beta \omega}}+\bar{\gamma} z_{1}-\bar{z}_{2} \gamma\right)}}{\left(1-e^{-\beta \omega}\right)^{2}} .
$$

These two operations, use of reproducing kernels and Gaußian integration for loops, can be tedious for more complicated graphs. This is the reason why we provide the general rules for the parametric representation of an arbitrary Feynman graph in subsection 3.5 .

In the last expression (4.5), only the term $\gamma=0$ is divergent. This divergence is local and quadratic in the cut-off $\left(\Lambda=\epsilon^{-1 / 2}\right)$, yielding a mass counterterm:

$$
\Gamma_{2 a, \mathrm{div}}^{(1)}\left[z_{1}, \bar{z}_{2}\right]=\frac{\lambda}{2} I\left(z_{1}, \bar{z}_{2}\right) \int_{\varepsilon}^{\infty} d \beta \frac{e^{-\frac{\beta \mu_{0}^{2}}{2}}}{\left(1-e^{-\beta \omega}\right)^{2}} .
$$

The (integrated) sum over $\gamma \neq 0$ is convergent as a distribution on $\varepsilon_{B}\left(\mathbb{C}^{2}\right)$, in the limit $\varepsilon \rightarrow 0$. (Recall that as a Fréchet space, $\mathcal{E}_{B}\left(\mathbb{C}^{2}\right)$ is defined as the image of $\varepsilon_{s}\left(\mathbb{R}^{2}\right)=\delta\left(\mathbb{R}^{2}\right)$ under the Bargmann transform.) Indeed, we have for any 
$\varphi_{1}, \varphi_{2} \in \mathcal{E}_{B}\left(\mathbb{C}^{2}\right)$ that

$$
\begin{aligned}
& \Gamma_{2 a, \mathrm{conv}}^{(1)}\left[\bar{\varphi}_{1}, \varphi_{2}\right]= \int d \mu\left(z_{1}, \bar{z}_{1}\right) d \mu\left(z_{2}, \bar{z}_{2}\right) \bar{\varphi}_{1}\left(\bar{z}_{1}\right) \varphi_{2}\left(z_{2}\right) I\left(z_{1}, \bar{z}_{2}\right) \\
& \cdot \int_{0}^{\infty} d \beta \sum_{\gamma \neq 0} \frac{e^{-\frac{\beta \mu_{0}^{2}}{2}} e^{-\omega\left(\frac{|\gamma|^{2}}{1-e^{-\beta \omega}}+\bar{\gamma} z_{1}-\bar{z}_{2} \gamma\right)}}{\left(1-e^{-\beta \omega}\right)^{2}} \\
&=\sum_{\gamma \neq 0}\left\langle\varphi_{1}, \varphi_{2} U_{\gamma}\right\rangle_{B} \int_{0}^{\infty} d \beta \frac{e^{-\frac{\beta \mu_{0}^{2}}{2}} e^{-\omega \frac{|\nu|^{2}}{2} \frac{1+e^{-\beta \omega}}{1-e^{-\beta \omega}}}}{\left(1-e^{-\beta \omega}\right)^{2}}
\end{aligned}
$$

The $\beta$-integral is bounded from above since $|\gamma|^{2} \geq \frac{\theta^{2}}{2}$. Because $\left\langle\varphi_{1}, \varphi_{2} U_{\gamma}\right\rangle_{B}$ is by construction a Schwartz sequence, the sum is finite. More precise estimations can be obtained using

$$
\frac{1-e^{-x_{0}}}{x_{0}} x \leq 1-e^{-x} \leq x \text { for } 0 \leq x \leq x_{0} .
$$

The second contribution to the 1-loop 2-point function is the graph

$$
\begin{aligned}
& \bar{z}_{2} z_{3}\left.\bar{z}_{4}\right\} z_{1} \Gamma_{2 b}^{(1)}\left[z_{1}, \bar{z}_{2}\right]=2 \int d \mu\left(z_{3}, \bar{z}_{3}\right) d \mu\left(z_{4}, \bar{z}_{3}\right) \\
& \cdot V\left(z_{1}, \bar{z}_{4}, z_{3}, \bar{z}_{2}\right) H_{\varepsilon}^{-1}\left(z_{4}, \bar{z}_{3}\right) .
\end{aligned}
$$

There are again two graphs of this type which after a change of variables give the same contribution. Using (3.70) and (3.66), we find

$$
\begin{aligned}
\Gamma_{2 b}^{(1)}\left[z_{1}, \bar{z}_{2}\right]= & \frac{\lambda}{2} I\left(z_{1}, \bar{z}_{2}\right) \sum_{\gamma} \int_{\varepsilon}^{\infty} d \beta e^{-\frac{\beta \mu_{0}^{2}}{2}} \\
& \cdot e^{-\omega\left(\bar{\gamma}\left(1-e^{-\beta \omega}\right) \gamma+\bar{\gamma}\left(1-e^{-\beta \omega}\right) z_{1}+\bar{z}_{2}\left(1-e^{-\beta \omega}\right) \gamma+\bar{z}_{2}\left(1-e^{-\beta \omega}\right) z_{1}\right)} .
\end{aligned}
$$

A small $\beta$ spoils the Gaußian decay for large $|\gamma|$. This can be made transparent by a Poisson re-summation. For that the convention on the Fourier transform we use is $\hat{f}(\xi)=\int e^{-2 i \pi x \xi} f(x) d x$. The formula we obtain with this convention is $\sum_{\gamma} e^{-\omega a|\gamma|^{2}} e^{-\omega(\bar{b} \gamma+\bar{\gamma} c)}=(a \theta)^{-2} \sum_{\gamma^{*}} e^{\frac{\omega}{a}\left(\bar{b}+i \bar{\gamma}^{*}\right)\left(c+i \gamma^{*}\right)}$ for all $a \in \mathbb{R}_{*}^{+}, b, c \in \mathbb{C}^{2}$, with the frequency $\omega=\frac{2 \pi}{\theta}$ and with $\gamma=\frac{\theta}{\sqrt{2}}(n+i m) \in \Gamma \subset \mathbb{C}^{2}$ one has $\gamma^{*}=$ $\frac{1}{\sqrt{2}}(n+i m) \in \hat{\Gamma} \subset \mathbb{C}^{2}$. In order to simplify the notation, here we use $\gamma^{*}$ instead of $\hat{\gamma}$ to denote an element of the dual lattice. 
Using this formula, we can continue the computation of (4.10):

$$
\Gamma_{2 b}^{(1)}\left[z_{1}, \bar{z}_{2}\right]=\frac{\lambda}{2 \theta^{2}} I\left(z_{1}, \bar{z}_{2}\right) \sum_{\gamma^{*}} \int_{\varepsilon}^{\infty} d \beta \frac{e^{-\frac{\beta \mu_{0}^{2}}{2}} e^{-\omega\left(\frac{\mid \gamma^{*}{ }^{2}}{\left(1-e^{-\beta \omega}\right)}-i \bar{z}_{2} \gamma^{*}-i \bar{\gamma}^{*} z_{1}\right)}}{\left(1-e^{-\beta \omega}\right)^{2}} .
$$

Thus the diagram (4.12) is, up to a normalisation factor $\theta^{-2}$ and the exchange $\gamma \leftrightarrow$ $i \gamma^{*}$, the same as (4.5): only the term with $\gamma^{*}=0$ is divergent, with a local quadratic divergence.

We have proven the following

Proposition 4.2. For any $\theta \in \mathbb{R}$, the divergent part of the 2-point 1 PI Green function associated with the action (2.33), in its 1-loop approximation, is given by

$$
\Gamma_{2, d i v}^{(1)}\left[\bar{\varphi}_{1}, \varphi_{2}\right]=\frac{\lambda}{2 \omega}\left(1+\frac{1}{\theta^{2}}\right) \operatorname{Tr}_{\mathcal{A}_{\theta}}\left[\left(\varphi_{1}, \varphi_{2}\right)_{\mathcal{A}_{\theta}}\right]\left(\frac{1}{\epsilon \omega}+\left(1-\frac{\mu_{0}^{2}}{2 \omega}\right) \ln \frac{1}{\epsilon}\right) .
$$

4.2. 4-point sector: planar graphs. Next we look at four-leg graphs. The first possibility is

$$
\bar{z}_{z_{1}}^{z_{3}}=-2 \int d \mu\left(z_{1}^{\prime}, \bar{z}_{1}^{\prime}\right) d \mu\left(z_{2}^{\prime}, \bar{z}_{2}^{\prime}\right) d \mu\left(z_{3}^{\prime}, \bar{z}_{3}^{\prime}\right) d \mu\left(z_{4}^{\prime}, \bar{z}_{4}^{\prime}\right)
$$

This graph appears four times in the expansion and because it contains two vertices there is a combinatorial factor $\frac{1}{2 !}$. The minus sign is from the Legendre transformation of 1PI functions. Three of the four integrations (e.g., over $z_{2}^{\prime}, z_{3}^{\prime}, z_{4}^{\prime}$ ) can be trivially done using the reproducing kernels in the vertex. Thus we have

$$
\begin{aligned}
& \Gamma_{4 a}^{(1)}\left[z_{1}, \bar{z}_{2}, z_{3}, \bar{z}_{4}\right] \\
& =-\frac{\lambda^{2}}{8} \int_{\varepsilon}^{\infty} d \beta_{1} d \beta_{2} \sum_{\gamma_{1}, \gamma_{2}} e^{-\frac{\mu_{0}^{2}}{2}\left(\beta_{1}+\beta_{2}\right)} e^{-\omega\left(\left|\gamma_{1}\right|^{2}+\left|\gamma_{2}\right|^{2}-\bar{z}_{2} \gamma_{1}-\bar{z}_{2} z_{1}+\bar{\gamma}_{1} z_{1}+\bar{z}_{4} \gamma_{2}-\bar{z}_{4} z_{3}-\bar{\gamma}_{2} z_{3}\right)} \\
& \cdot \int d \mu(z, \bar{z}) e^{-\omega\left(-\bar{z} e^{-\beta_{1} \omega} \gamma_{2}+\bar{z} e^{-\left(\beta_{1}+\beta_{2}\right) \omega} \gamma_{1}-\bar{\gamma}_{2} e^{-\beta_{2} \omega} \gamma_{1}-\bar{z} e^{-\left(\beta_{1}+\beta_{2}\right) \omega} z+\bar{\gamma}_{2} e^{-\beta_{2} \omega} z-\bar{\gamma}_{1} z\right)}
\end{aligned}
$$


We integrate over $z$, put $\gamma_{2}=\gamma_{1}+\gamma$ and obtain after some reorganisations

$$
\begin{aligned}
& \Gamma_{4 a}^{(1)}\left[z_{1}, \bar{z}_{2}, z_{3}, \bar{z}_{4}\right] \\
& =-\frac{\lambda^{2}}{8} \sum_{\gamma_{1}, \gamma} e^{-\omega\left|\gamma_{1}\right|^{2}} I\left(\gamma_{1}, \bar{z}_{2}-\bar{z}_{4}\right) I\left(z_{1}, \bar{z}_{2}-\bar{\gamma}_{1}\right) I\left(z_{3}, \bar{z}_{4}+\bar{\gamma}_{1}\right) \\
& \int_{\varepsilon}^{\infty} \frac{d \beta_{1} d \beta_{2} e^{-\frac{\mu_{0}^{2}}{2}\left(\beta_{1}+\beta_{2}\right)}}{\left(1-e^{-\left(\beta_{1}+\beta_{2}\right) \omega}\right)^{2}}
\end{aligned}
$$

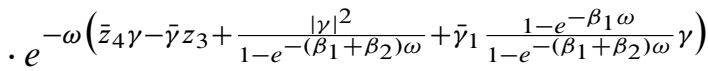

$$
\begin{aligned}
& \cdot e^{-\omega\left(\bar{\gamma} \frac{1-e^{-\beta_{2} \omega}}{1-e^{-\left(\beta_{1}+\beta_{2}\right) \omega}} \gamma_{1}+\left|\gamma_{1}\right|^{2} \frac{\left(1-e^{-\beta_{1} \omega}\right)\left(1-e^{-\beta_{2} \omega}\right)}{1-e^{-\left(\beta_{1}+\beta_{2}\right) \omega}}\right)} .
\end{aligned}
$$

For $\gamma=0$ one obtains

$$
\begin{aligned}
\Gamma_{4 a, 0}^{(1)}\left[z_{1}, \bar{z}_{2}, z_{3}, \bar{z}_{4}\right] & \\
= & -\frac{\lambda}{4} V\left(z_{1}, \bar{z}_{2}, z_{3}, \bar{z}_{4}\right) \int_{\varepsilon}^{\infty} \frac{d \beta_{1} d \beta_{2} e^{-\frac{\mu_{0}^{2}}{2}\left(\beta_{1}+\beta_{2}\right)}}{\left(1-e^{-\left(\beta_{1}+\beta_{2}\right) \omega}\right)^{2}} \\
& +\frac{\lambda^{2}}{8} \sum_{\gamma_{1}} e^{-\omega\left|\gamma_{1}\right|^{2}} I\left(\gamma_{1}, \bar{z}_{2}-\bar{z}_{4}\right) I\left(z_{1}, \bar{z}_{2}-\bar{\gamma}_{1}\right) I\left(z_{3}, \bar{z}_{4}+\bar{\gamma}_{1}\right) \\
& \cdot \int_{\varepsilon}^{\infty} \frac{d \beta_{1} d \beta_{2} e^{-\frac{\mu_{0}^{2}}{2}\left(\beta_{1}+\beta_{2}\right)}}{\left(1-e^{-\left(\beta_{1}+\beta_{2}\right) \omega}\right)^{2}}\left(1-e^{\left.-\omega\left|\gamma_{1}\right|^{2} \frac{\left(1-e^{-\beta_{1} \omega}\right)\left(1-e^{-\beta_{2} \omega}\right)}{1-e^{-\left(\beta_{1}+\beta_{2}\right) \omega}}\right) .}\right.
\end{aligned}
$$

The line (4.15) gives the logarithmically divergent renormalisation of the coupling constant. It remains to show that the remaining parts are convergent. In (4.16), if we add the external fields $\varphi_{1}, \varphi_{2}, \varphi_{3}, \varphi_{4} \in \mathcal{E}_{B}\left(\mathbb{C}^{2}\right)$ and if use the inequality (4.8), we obtain

$$
\begin{aligned}
\mid \sum_{\gamma_{1}}\left\langle\varphi_{1}, \varphi_{2} U_{\gamma_{1}}\right\rangle_{B}\left\langle\varphi_{3}, \varphi_{4} U_{-\gamma_{1}}\right\rangle_{B} \\
\quad \cdot \int_{0}^{\infty} \frac{d \beta_{1} d \beta_{2} e^{-\frac{\mu_{0}^{2}}{2}\left(\beta_{1}+\beta_{2}\right)}}{\left(1-e^{\left.-\left(\beta_{1}+\beta_{2}\right) \omega\right)^{2}}\right.}\left(1-e^{\left.-\omega\left|\gamma_{1}\right|^{2} \frac{\left(1-e^{-\beta_{1} \omega}\right)\left(1-e^{-\beta_{2} \omega}\right)}{1-e^{-\left(\beta_{1}+\beta_{2}\right) \omega}}\right) \mid}\right. \\
\leq \omega \sum_{\gamma_{1}}\left|\left\langle\varphi_{1}, \varphi_{2} U_{\gamma_{1}}\right\rangle_{B}\right|\left|\left\langle\varphi_{3}, \varphi_{4} U_{-\gamma_{1}}\right\rangle_{B}\right|\left|\gamma_{1}\right|^{2} \\
\quad \cdot \int_{0}^{\infty} d \beta_{1} d \beta_{2} e^{-\frac{\mu_{0}^{2}}{2}\left(\beta_{1}+\beta_{2}\right)} \frac{\left(1-e^{-\beta_{1} \omega}\right)\left(1-e^{-\beta_{2} \omega}\right)}{\left(1-e^{-\left(\beta_{1}+\beta_{2}\right) \omega}\right)^{3}},
\end{aligned}
$$

which is finite since $\left\langle\varphi_{i}, \varphi_{j} U_{\gamma_{1}}\right\rangle_{B}$ is a Schwartz sequence and because the $\beta$-integral is now harmless. 
It remains to estimate in (4.14) the remaining sum over $\gamma \neq 0$. Similar manipulations show that

$\Gamma_{4 a, \neq 0}^{(1)}\left[\bar{\varphi}_{1}, \varphi_{2}, \bar{\varphi}_{3}, \varphi_{4}\right]$

$$
\begin{aligned}
&=-\frac{\lambda^{2}}{8} \sum_{\gamma_{1}, \gamma \neq 0}\left\langle\varphi_{1}\right.\left.\varphi_{2} U_{-\gamma_{1}}\right\rangle_{B}\left\langle\varphi_{3}, \varphi_{4} U_{\gamma_{1}}+\gamma\right\rangle_{B} \int_{0}^{\infty} \frac{d \beta_{1} d \beta_{2} e^{-\frac{\mu_{0}^{2}}{2}\left(\beta_{1}+\beta_{2}\right)}}{\left(1-e^{-\left(\beta_{1}+\beta_{2}\right) \omega}\right)^{2}} \\
& \cdot e^{-\frac{\omega}{2}\left(|\gamma|^{2} \frac{1+e^{-\left(\beta_{1}+\beta_{2}\right) \omega}}{\left.1-e^{-\left(\beta_{1}+\beta_{2}\right) \omega}+\bar{\gamma}_{1} \gamma \frac{1-2 e^{-\beta_{1} \omega}+e^{-\left(\beta_{1}+\beta_{2}\right) \omega}}{1-e^{-\left(\beta_{1}+\beta_{2}\right) \omega}}\right)}\right.} \\
& \cdot e^{-\frac{\omega}{2}\left(\bar{\gamma} \gamma_{1} \frac{1-2 e^{-\beta_{2} \omega}+e^{-\left(\beta_{1}+\beta_{2}\right) \omega}}{1-e^{-\left(\beta_{1}+\beta_{2}\right) \omega}}+2\left|\gamma_{1}\right|^{2} \frac{\left(1-e^{-\beta_{1} \omega}\right)\left(1-e^{-\beta_{2} \omega}\right)}{1-e^{-\left(\beta_{1}+\beta_{2}\right) \omega}}\right.} .
\end{aligned}
$$

Thus,

$$
\begin{aligned}
& \left|\Gamma_{4 a, \neq 0}^{(1)}\left[\bar{\varphi}_{1}, \varphi_{2}, \bar{\varphi}_{3}, \varphi_{4}\right]\right| \\
& \leq \frac{\lambda^{2}}{8} \sum_{\gamma_{1}, \gamma \neq 0}\left|\left\langle\varphi_{1}, \varphi_{2} U_{-\gamma_{1}}\right\rangle_{B}\left\langle\varphi_{3}, \varphi_{4} U_{\gamma_{1}+\gamma}\right\rangle_{B}\right| \int_{0}^{\infty} \frac{d \beta_{1} d \beta_{2} e^{-\frac{\mu_{0}^{2}}{2}\left(\beta_{1}+\beta_{2}\right)}}{\left(1-e^{-\left(\beta_{1}+\beta_{2}\right) \omega}\right)^{2}}
\end{aligned}
$$

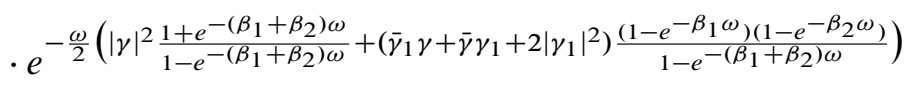

$$
\begin{aligned}
& \leq \frac{\lambda^{2}}{8} \sum_{\gamma_{1}, \gamma \neq 0}\left|\left\langle\varphi_{1}, \varphi_{2} U_{-\gamma_{1}}\right\rangle_{B}\left\langle\varphi_{3}, \varphi_{4} U_{\gamma_{1}+\gamma}\right\rangle_{B}\right| \\
& \cdot \int_{0}^{\infty} \frac{d \beta_{1} d \beta_{2} e^{-\frac{\mu_{0}^{2}}{2}\left(\beta_{1}+\beta_{2}\right)}}{\left(1-e^{-\left(\beta_{1}+\beta_{2}\right) \omega}\right)^{2}} e^{-\frac{\omega \theta^{2}}{4} \frac{e^{-\beta_{1} \omega}+e^{-\beta_{2} \omega}}{1-e^{-\left(\beta_{1}+\beta_{2}\right) \omega}}}
\end{aligned}
$$

which is finite.

The next graph is

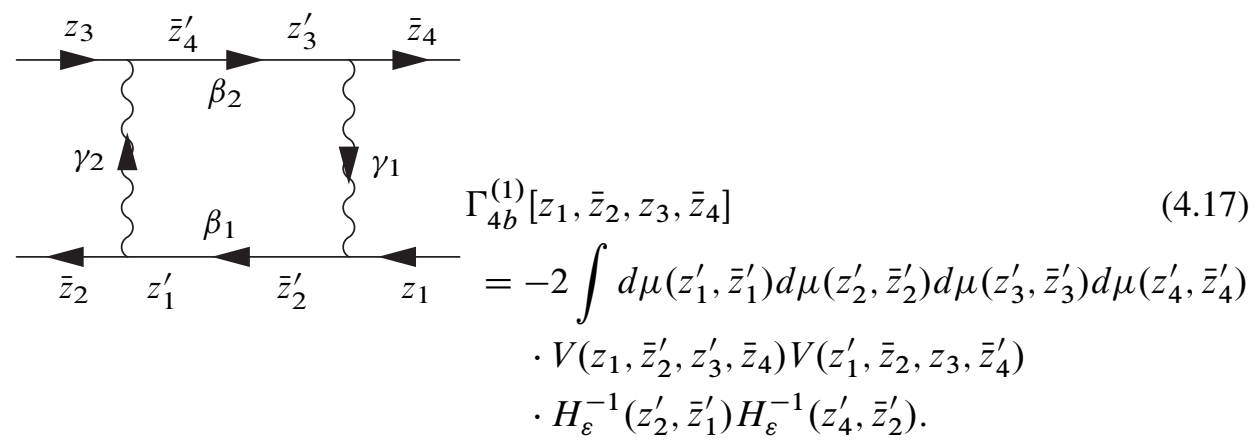

This graph appears with the same symmetry factor as the first one. All $z^{\prime}$-integrations 
can be trivially done using the reproducing kernels in the vertices, giving

$$
\begin{array}{r}
\Gamma_{4 b}^{(1)}\left[z_{1}, \bar{z}_{2}, z_{3}, \bar{z}_{4}\right] \\
=-\frac{\lambda^{2}}{8} \sum_{\gamma_{1}, \gamma_{2}} \int_{\varepsilon}^{\infty} d \beta_{1} d \beta_{2} e^{-\frac{\mu_{0}^{2}}{2}\left(\beta_{1}+\beta_{2}\right)} e^{-\omega\left(\left|\gamma_{2}\right|^{2}+\left|\gamma_{1}\right|^{2}+\bar{z}_{2} \gamma_{2}+\bar{\gamma}_{1} z_{1}+\bar{z}_{4} \gamma_{1}+\bar{\gamma}_{2} z_{3}\right)} \\
\cdot e^{\omega\left(\bar{z}_{2} e^{-\beta_{1} \omega} \gamma_{1}+\bar{\gamma}_{2} e^{-\beta_{1} \omega} \gamma_{1}+\bar{z}_{2} e^{-\beta_{1} \omega} z_{1}+\bar{\gamma}_{2} e^{-\beta_{1} \omega} z_{1}\right)} \\
\cdot e^{\omega\left(\bar{z}_{4} e^{-\beta_{2} \omega} \gamma_{2}+\bar{\gamma}_{1} e^{-\beta_{2} \omega} \gamma_{2}+\bar{z}_{4} e^{-\beta_{2} \omega} z_{3}+\bar{\gamma}_{1} e^{-\beta_{2} \omega} z_{3}\right)} .
\end{array}
$$

There are two ways to proceed. We could set $\gamma_{1}=\gamma_{2}+\gamma$ and perform a Poisson resummation (4.11) in the loop variable $\gamma_{2}$. The other possibility, which underlines the duality with the previous graph, consists in a Poisson re-summation in both $\gamma_{1}$ and $\gamma_{2}$ :

$$
\begin{aligned}
& \Gamma_{4 b}^{(1)}\left[z_{1}, \bar{z}_{2}, z_{3}, \bar{z}_{4}\right] \\
& =-\frac{\lambda^{2}}{8 \theta^{4}} e^{\omega\left(\bar{z}_{4} z_{1}+\bar{z}_{2} z_{3}\right)} \sum_{\gamma_{1}^{*}, \gamma_{2}^{*}} \int_{\varepsilon}^{\infty} d \beta_{1} d \beta_{2} \frac{e^{-\frac{\mu_{0}^{2}}{2}\left(\beta_{1}+\beta_{2}\right) \omega}}{\left(1-e^{-\left(\beta_{1}+\beta_{2}\right) \omega}\right)^{2}}
\end{aligned}
$$

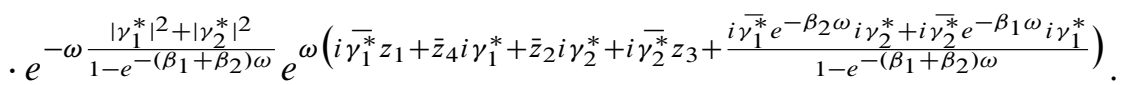

If we plug now $\gamma_{2}^{*}=-\gamma_{1}^{*}+\gamma^{*}$, we see that this graph leads to the same expression as the previous one (4.14), up to a constant factor and the exchange $\gamma_{1} \rightarrow i \gamma_{1}^{*}$, $\gamma \rightarrow-i \gamma^{*}$ and $z_{2} \leftrightarrow z_{4}$ :

$$
\begin{aligned}
& \Gamma_{4 b}^{(1)}\left[z_{1}, \bar{z}_{2}, z_{3}, \bar{z}_{4}\right] \\
& =-\frac{\lambda^{2}}{8 \theta^{4}} \sum_{\gamma_{1}^{*}, \gamma^{*}} e^{-\omega\left|\gamma_{1}^{*}\right|^{2}} I\left(i \gamma_{1}^{*}, \bar{z}_{4}-\bar{z}_{2}\right) I\left(z_{1}, \bar{z}_{4}-\overline{i \gamma_{1}^{*}}\right) I\left(z_{3}, \bar{z}_{2}+\overline{i \gamma_{1}^{*}}\right) \\
& \cdot \int_{\varepsilon}^{\infty} \frac{d \beta_{1} d \beta_{2} e^{-\frac{\mu_{0}^{2}}{2}\left(\beta_{1}+\beta_{2}\right)}}{\left(1-e^{-\left(\beta_{1}+\beta_{2}\right) \omega}\right)^{2}} e^{-\omega\left(-\bar{z}_{2} i \gamma^{*}-i \bar{\gamma}^{*} z_{3}+\frac{\left|\gamma^{*}\right|^{2}}{1-e^{-\left(\beta_{1}+\beta_{2}\right) \omega}}\right)}
\end{aligned}
$$

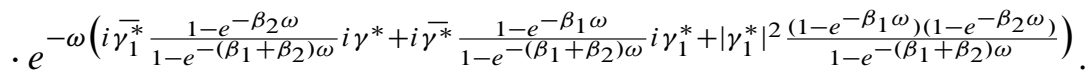

The divergent part is thus given by

$$
\begin{aligned}
\Gamma_{4 b, \mathrm{div}}^{(1)}\left[z_{1}, \bar{z}_{2}, z_{3}, \bar{z}_{4}\right] & \\
= & -\frac{\lambda^{2}}{8 \theta^{4}} \sum_{\gamma_{1}^{*}} e^{-\omega\left|\gamma_{1}^{*}\right|^{2}} I\left(i \gamma_{1}^{*}, \bar{z}_{4}-\bar{z}_{2}\right) I\left(z_{1}, \bar{z}_{4}-\overline{i \gamma_{1}^{*}}\right) \\
& \cdot I\left(z_{3}, \bar{z}_{2}+\overline{i \gamma_{1}^{*}}\right) \int_{\varepsilon}^{\infty} \frac{d \beta_{1} d \beta_{2} e^{-\frac{\mu_{0}^{2}}{2}\left(\beta_{1}+\beta_{2}\right)}}{\left(1-e^{-\left(\beta_{1}+\beta_{2}\right) \omega}\right)^{2}}
\end{aligned}
$$

which gives after a (final) Poisson re-summation (4.11) another (local, logarithmically divergent) contribution for the coupling constant renormalisation: 


$$
\Gamma_{4 b, \mathrm{div}}^{(1)}\left[z_{1}, \bar{z}_{2}, z_{3}, \bar{z}_{4}\right]=-\frac{\lambda}{4 \theta^{2}} V\left(z_{1}, \bar{z}_{2}, z_{3}, \bar{z}_{4}\right) \int_{\varepsilon}^{\infty} \frac{d \beta_{1} d \beta_{2} e^{-\frac{\mu_{0}^{2}}{2}\left(\beta_{1}+\beta_{2}\right)}}{\left(1-e^{-\left(\beta_{1}+\beta_{2}\right) \omega}\right)^{2}} .
$$

We can summarise these estimations in the following

Proposition 4.3. For any $\theta \in \mathbb{R}$, the divergent part of the 4-point planar 1PI Green function associated with the action (2.33), in its 1-loop approximation, is given by

$$
\Gamma_{4, \text { planar,div }}^{(1)}\left[\bar{\varphi}_{1}, \varphi_{2}, \bar{\varphi}_{3}, \varphi_{3}\right]=-\frac{\lambda^{2}}{8 \omega^{2}}\left(1+\frac{1}{\theta^{2}}\right) \operatorname{Tr}_{\mathcal{A}_{\theta}}\left[\left(\varphi_{1}, \varphi_{2}\right)_{\mathcal{A}_{\theta}}\left(\varphi_{3}, \varphi_{4}\right)_{\mathcal{A}_{\theta}}\right] \ln \frac{1}{\epsilon} .
$$

4.3. 4-point sector: non-planar graphs. Up to now we have found only local divergences which can be absorbed in a mass and coupling constant renormalisation. This is not the end of the story. We will see that the next two 4-point 1-loop graphs develop non-local divergences in the sense that the counterterm needed is not present in the classical action (2.33). Indeed, the next graphs are topologically non-planar and their singularities are of the same kind as the singularity of the non-planar sector of a scalar field theory on the noncommutative torus or even on certain isospectral deformations [16]. This is the discrete version of the so-called the UV/IR-entanglement phenomenon: these new singularities are restricted to the zero-mode $\gamma=0$ of the vertex. They should not appear in models where the zero-mode can be consistently removed such as for U(1)-Yang-Mills theory on the noncommutative torus [23].

As we will see, the behaviour of these non-planar graphs for the Heisenberg module is highly sensitive to the number theoretical aspect of the deformation parameter $\theta$. There are two different cases to consider. When $\theta$ is rational, we know that the model is a commutative one and the singularities are local. For irrational $\theta$, in order to control the sums for non-vanishing $\gamma$, we need to impose a Diophantine type condition. We first give definitions.

Definition 4.4. An irrational number $\theta$ is said to satisfy a Diophantine condition if for all $n \in \mathbb{Z} \backslash\{0\}$ there exist two constants $C, \delta>0$ such that

$$
\|n \theta\|_{\mathbb{T}}=\inf _{m \in \mathbb{Z}}|n \theta-m| \geq C|n|^{-(1+\delta)} .
$$

We have the possibility to weaken this condition: An irrational number $\theta$ is said to satisfy a weak Diophantine condition if for all $n \in \mathbb{Z} \backslash\{0\}$ there exist two constants $C_{1}, C_{2}>0$ such that

$$
\|n \theta\|_{\mathbb{T}}=\inf _{m \in \mathbb{Z}}|n \theta-m| \geq C_{1} e^{-C_{2}|n|} .
$$

These definitions extend to tuples of irrational number $\left(\theta_{1}, \ldots, \theta_{d}\right)$, where the exponent $1+\delta$ in (4.21) has to be replaced by $d+\delta$. 
A Diophantine condition is a way to measure "how far from rationals" is an irrational number. More precisely, it means that the convergence of the continuous fractional expansion is quite slow. Note that the (ordinary) Diophantine condition is equivalent to the requirement that the inverse torus norm of $n \theta$ is a tempered distribution on $\delta(\mathbb{Z} \backslash\{0\})$. This is a natural requirement because of the distributional nature of QFT and since the basic formulation of our model relies on Schwartz sequences. Note also that irrational numbers satisfying a Diophantine condition are not exceptional since they are of full Lebesgue measure. However, since at 1-loop the non-planar sector is only logarithmically divergent, this condition can be weakened: we are able to control the convergence even under the weakest version of the Diophantine condition (4.22).

The first non-planar graph to compute is given by

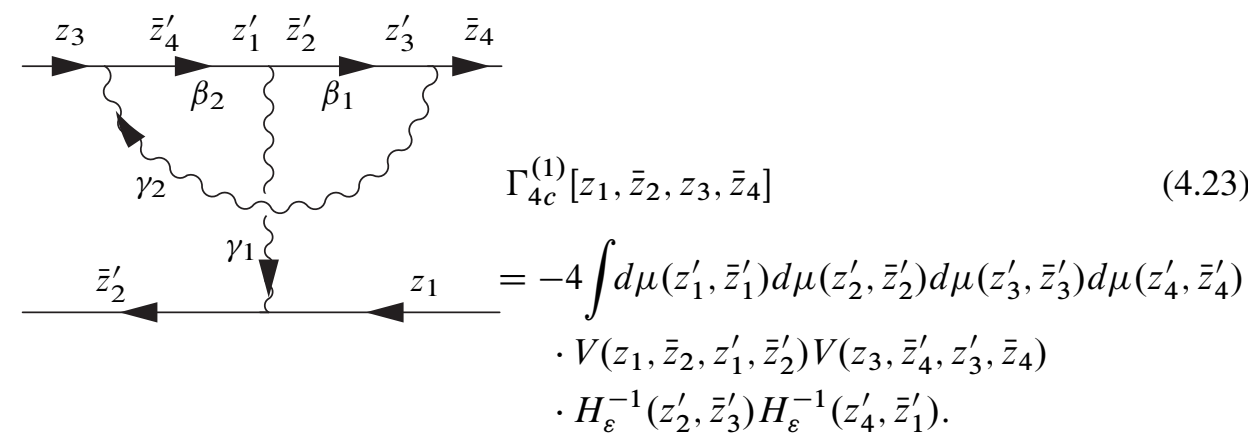

This graph appears eight times in the expansion, leading to twice the symmetry factor of the previous graphs. We have

$$
\begin{aligned}
\Gamma_{4 c}^{(1)} & {\left[z_{1}, \bar{z}_{2}, z_{3}, \bar{z}_{4}\right] } \\
= & -\frac{\lambda^{2}}{4} \sum_{\gamma_{1}, \gamma_{2}} \int_{\varepsilon}^{\infty} d \beta_{1} d \beta_{2} e^{-\frac{\mu_{0}^{2}}{2}\left(\beta_{1}+\beta_{2}\right)} e^{-\omega\left(\left|\gamma_{1}\right|^{2}+\left|\gamma_{2}\right|^{2}-\bar{z}_{2} \gamma_{1}-\left(\bar{z}_{2}-\bar{\gamma}_{1}\right) z_{1}+\bar{z}_{4} \gamma_{2}\right)} \\
& \cdot e^{\omega\left(-\left(\bar{z}_{4}+\bar{\gamma}_{2}\right) e^{-\beta_{1} \omega} \gamma_{1}+\left(\left(\bar{z}_{4}+\bar{\gamma}_{2}\right) e^{-\left(\beta_{1}+\beta_{2}\right) \omega}+\bar{\gamma}_{1} e^{-\beta_{2} \omega}\right) \gamma_{2}\right)} \\
& \cdot e^{\omega\left(\left(\left(\bar{z}_{4}+\bar{\gamma}_{2}\right) e^{-\left(\beta_{1}+\beta_{2}\right) \omega}+\bar{\gamma}_{1} e^{-\beta_{2} \omega}-\bar{\gamma}_{2}\right) z_{3}\right)} .
\end{aligned}
$$

We perform a Poisson re-summation in the loop wavy line index (in $\left.\gamma_{2}\right)$ :

$$
\begin{aligned}
& \Gamma_{4 c}^{(1)}\left[z_{1}, \bar{z}_{2}, z_{3}, \bar{z}_{4}\right]=-\frac{\lambda^{2}}{4 \theta^{2}} \sum_{\gamma_{1}, \gamma_{2}^{*}} e^{\omega\left(\bar{z}_{2} z_{1}+\bar{z}_{4} z_{3}+i \bar{\gamma}_{2}^{*} z_{3}+\bar{z}_{4} i \gamma_{2}^{*}-\bar{\gamma}_{1} z_{1}+\bar{z}_{2} \gamma_{1}\right)} \\
& \cdot \int_{\varepsilon}^{\infty} d \beta_{1} d \beta_{2} \frac{e^{-\frac{\mu_{0}^{2}}{2}\left(\beta_{1}+\beta_{2}\right)}}{\left(1-e^{-\left(\beta_{1}+\beta_{2}\right) \omega}\right)^{2}} \\
& \cdot e^{-\frac{\omega}{1-e^{-\left(\beta_{1}+\beta_{2}\right) \omega}}\left(\left|\gamma_{1}\right|^{2}+\left|\gamma_{2}^{*}\right|^{2}+\bar{\gamma}_{1} i \gamma_{2}^{*} e^{-\beta_{2} \omega}-i \gamma_{2}^{*} \gamma_{1} e^{-\beta_{1} \omega}\right)} .
\end{aligned}
$$


In order to see what happens in the different arithmetical cases, it is perhaps more enlightening to add external field $\varphi_{1}, \varphi_{2}, \varphi_{3}, \varphi_{4} \in \mathcal{E}_{B}$. After some rearrangements, we obtain

$$
\begin{aligned}
& \Gamma_{4 c}^{(1)}\left[\bar{\varphi}_{1}, \varphi_{2}, \bar{\varphi}_{3}, \varphi_{4}\right] \\
& =-\frac{\lambda^{2}}{4 \theta^{2}} \sum_{\gamma_{1}, \gamma_{2}^{*}}\left\langle\varphi_{1}, \varphi_{2} U_{\gamma_{1}}\right\rangle_{B}\left\langle\varphi_{3}, \varphi_{4} U_{\left.i \gamma_{2}^{*}\right\rangle_{B}}\right. \\
& \quad \cdot \int_{\varepsilon}^{\infty} d \beta_{1} d \beta_{2} \frac{e^{-\frac{\mu_{0}^{2}}{2}\left(\beta_{1}+\beta_{2}\right)}}{\left(1-e^{-\left(\beta_{1}+\beta_{2}\right) \omega}\right)^{2}} e^{-\frac{\omega}{2} \frac{1+e^{-\left(\beta_{1}+\beta_{2}\right) \omega}}{1-e^{-\left(\beta_{1}+\beta_{2}\right) \omega}\left(\left|\gamma_{1}\right|^{2}+\left|\gamma_{2}^{*}\right|^{2}\right)}} \\
& \cdot e^{-\frac{\omega}{1-e^{-\left(\beta_{1}+\beta_{2}\right) \omega}}\left(\bar{\gamma}_{1} i \gamma_{2}^{*} e^{-\beta_{2} \omega}-i \gamma_{2}^{*} \gamma_{1} e^{-\beta_{1} \omega}\right)}
\end{aligned}
$$

Now the number theoretical aspect of the deformation parameter $\theta$ comes into the play. Since $\gamma^{*}=\frac{1}{\sqrt{2}}(m+i n)$, for $\theta \in \mathbb{N}$ (commutative case) we can set $\gamma_{1}=-i \gamma_{2}^{*}$ in (4.25) (by estimates similar to those used in this section, the sum $\gamma_{1} \neq-i \gamma_{2}^{*}$ can be shown to give a finite contribution) and obtain

$$
\begin{aligned}
-\frac{\lambda^{2}}{4 \theta^{2}} \sum_{\gamma_{1}}\left\langle\varphi_{1}, \varphi_{2} U_{\gamma_{1}}\right\rangle_{B}\left\langle\varphi_{3}, \varphi_{4} U_{-\gamma_{1}}\right\rangle_{B} \\
\quad \int_{\varepsilon}^{\infty} d \beta_{1} d \beta_{2} \frac{e^{-\frac{\mu_{0}^{2}}{2}\left(\beta_{1}+\beta_{2}\right)}}{\left(1-e^{-\left(\beta_{1}+\beta_{2}\right) \omega}\right)^{2}} e^{-\omega\left|\gamma_{1}\right|^{2} \frac{\left(1-e^{-\beta_{1} \omega}\right)\left(1-e^{-\beta_{2} \omega}\right)}{1-e^{-\left(\beta_{1}+\beta_{2}\right) \omega}}} .
\end{aligned}
$$

Thus, using the routine inequalities, we can now extract the divergent part of $\Gamma_{4 d}^{(1)}\left[z_{1}, \bar{z}_{2}, z_{3}, \bar{z}_{4}\right]$ :

$$
\Gamma_{4 c, \text { div }}^{(1)}\left[z_{1}, \bar{z}_{2}, z_{3}, \bar{z}_{4}\right]=-\frac{\lambda}{2 \theta^{2}} V\left(z_{1}, \bar{z}_{2}, z_{3}, \bar{z}_{4}\right) \int_{\varepsilon}^{\infty} d \beta_{1} d \beta_{2} \frac{e^{-\frac{\mu_{0}^{2}}{2}\left(\beta_{1}+\beta_{2}\right)}}{\left(1-e^{-\left(\beta_{1}+\beta_{2}\right) \omega}\right)^{2}},
$$

so in the commutative case the divergence is local as expected.

When $\theta$ is rational, the divergence is local as well. Indeed, for $\theta=p / q$ we have $\gamma_{1}=\frac{p}{q \sqrt{2}}\left(m_{1}+i n_{1}\right)$ and $-i \gamma_{2}^{*}=\frac{1}{\sqrt{2}}\left(n_{2}-i m_{2}\right)$. Thus, if we restrict the sum to the set of $\left(m_{1}, n_{1}, m_{2}, n_{2}\right) \in \mathbb{Z}^{8}$ such that $p n_{1}=-q m_{2}$ and $p m_{1}=q n_{2}$ (the rest is a finite sum that corresponds to a finite multiplicity and yields a finite contribution) and if we add external fields and use the transformation (3.36), we find for the divergent part:

$$
-\frac{q^{2} \lambda^{2}}{4 p^{2}} \int_{0}^{\frac{1}{q}} d x \int_{0}^{\frac{1}{q}} d y \operatorname{Tr}\left[M^{\dagger} M\right](x, y) \operatorname{Tr}\left[M^{\dagger} M\right](x, y) \int_{\varepsilon}^{\infty} d \beta_{1} d \beta_{2} \frac{e^{-\frac{\mu_{0}^{2}}{2}\left(\beta_{1}+\beta_{2}\right)}}{\left(1-e^{-\left(\beta_{1}+\beta_{2}\right) \omega}\right)^{2}} .
$$

Whereas unfamiliar in the standard formulation of a field theory on a vector bundle with matricial fibres, such a term in the original action is perfectly acceptable. 
When $\theta$ is irrational and satisfies a Diophantine condition (Definition 4.4) to control the sums $\gamma_{1}, \gamma_{2}^{*} \neq 0$, we see in (4.25) that only the mode $\gamma_{1}=0, \gamma_{2}^{*}=0$ will contribute to the divergent part. There are three regions to consider:

$$
\text { (I) } \gamma_{1}=\gamma_{2}^{*}=0, \quad \text { (II) } \gamma_{1}=0, \gamma_{2}^{*} \neq 0, \quad \text { (III) } \gamma_{1} \neq 0 \text {, any } \gamma_{2}^{*} \text {. }
$$

In case (I), we obtain

$$
\Gamma_{4 c, \theta \notin \mathbb{Q},(\mathrm{I})}^{(1)}\left[\bar{\varphi}_{1}, \varphi_{2}, \bar{\varphi}_{3}, \varphi_{4}\right]=-\frac{\lambda^{2}}{4 \theta^{2}}\left\langle\varphi_{1}, \varphi_{2}\right\rangle_{B}\left\langle\varphi_{3}, \varphi_{4}\right\rangle_{B} \int_{\varepsilon}^{\infty} d \beta_{1} d \beta_{2} \frac{e^{-\frac{\mu_{0}^{2}}{2}\left(\beta_{1}+\beta_{2}\right)}}{\left(1-e^{-\left(\beta_{1}+\beta_{2}\right) \omega}\right)^{2}} .
$$

It is worthwhile to notice that this divergence is non-local and requires a counterterm of the form

$$
\operatorname{Tr}_{\mathcal{A}_{\theta}}\left[(\varphi, \varphi)_{\mathcal{A}_{\theta}}\right] \operatorname{Tr}_{\mathcal{A}_{\theta}}\left[(\varphi, \varphi)_{\mathcal{A}_{\theta}}\right] .
$$

This is the discrete version of the UV/IR-entanglement, reminiscent of toric-noncommutative spaces [16].

The estimation in region (II) is analogous to an estimation we performed for planar graphs. It remains to estimate the region (III), where the Diophantine condition will be used. We obtain in the limit $\varepsilon \rightarrow 0$ :

$$
\begin{aligned}
& \left|\Gamma_{4 c, \theta \notin \mathbb{Q},(\mathrm{III})}^{(1)}\left[\bar{\varphi}_{1}, \varphi_{2}, \bar{\varphi}_{3}, \varphi_{4}\right]\right| \\
& \leq \frac{\lambda^{2}}{4 \theta^{2}} \sum_{\gamma_{1} \neq 0, \gamma_{2}^{*}}\left|\left\langle\varphi_{1}, \varphi_{2} U_{\gamma_{1}}\right\rangle_{B}\right|\left|\left\langle\varphi_{3}, \varphi_{4} U_{i \gamma_{2}^{*}}\right\rangle_{B}\right| \\
& \cdot \int_{0}^{\infty} d \beta_{1} d \beta_{2} \frac{e^{-\frac{\mu_{0}^{2}}{2}\left(\beta_{1}+\beta_{2}\right)}}{\left(1-e^{-\left(\beta_{1}+\beta_{2}\right) \omega}\right)^{2}} e^{-\frac{\omega}{2} \frac{1+e^{-\left(\beta_{1}+\beta_{2}\right) \omega}}{1-e^{-\left(\beta_{1}+\beta_{2}\right) \omega}}\left(\left|\gamma_{1}\right|^{2}+\left|\gamma_{2}^{*}\right|^{2}\right)} \\
& e^{-\frac{\omega}{2} \frac{e^{-\beta_{1}+e^{-\beta_{2}}}}{1-e^{-\left(\beta_{1}+\beta_{2}\right) \omega}\left(\bar{\gamma}_{1} i \gamma_{2}^{*}-i \overline{\gamma_{2}^{*}} \gamma_{1}\right)}} \\
& =\frac{\lambda^{2}}{4 \theta^{2}} \sum_{\gamma_{1} \neq 0, \gamma_{2}^{*}}\left|\left\langle\varphi_{1}, \varphi_{2} U_{\gamma_{1}}\right\rangle_{B}\right|\left|\left\langle\varphi_{3}, \varphi_{4} U_{i \gamma_{2}^{*}}\right\rangle_{B}\right| \\
& \cdot \int_{0}^{\infty} d \beta_{1} d \beta_{2} \frac{e^{-\frac{\mu_{0}^{2}}{2}\left(\beta_{1}+\beta_{2}\right)}}{\left(1-e^{-\left(\beta_{1}+\beta_{2}\right) \omega}\right)^{2}} e^{-\frac{\omega}{2} \frac{e^{-\beta_{1} \omega}+e^{-\beta_{2} \omega}}{1-e^{-\left(\beta_{1}+\beta_{2}\right) \omega}}\left|\gamma_{1}+i \gamma_{2}^{*}\right|^{2}} \\
& e^{-\frac{\omega}{2} \frac{\left(1-e^{\left.-\beta_{1}\right)\left(1-e^{-\beta_{2}}\right.}\right.}{1-e^{-\left(\beta_{1}+\beta_{2}\right) \omega}}\left(\left|\gamma_{1}\right|^{2}+\left|\gamma_{2}^{*}\right|^{2}\right)} \\
& \leq \frac{\lambda^{2}}{4 \theta^{2}} \sum_{\gamma_{1} \neq 0, \gamma_{2}^{*}}\left|\left\langle\varphi_{1}, \varphi_{2} U_{\gamma_{1}}\right\rangle_{B}\right|\left|\left\langle\varphi_{3}, \varphi_{4} U_{i \gamma_{2}^{*}}\right\rangle_{B}\right| \\
& \cdot \int_{0}^{\infty} d \beta_{1} d \beta_{2} \frac{e^{-\frac{\mu_{0}^{2}}{2}\left(\beta_{1}+\beta_{2}\right)}}{\left(1-e^{-\left(\beta_{1}+\beta_{2}\right) \omega}\right)^{2}} e^{-\frac{\omega}{2} \frac{e^{-\beta_{1} \omega}+e^{-\beta_{2} \omega}}{1-e^{-\left(\beta_{1}+\beta_{2}\right) \omega}\left|\gamma_{1}+i \gamma_{2}^{*}\right|^{2}}} .
\end{aligned}
$$


Using now the (regular) Diophantine condition according to Definition 4.4, and disregarding the $\beta$-integrals from 1 to $+\infty$ (which gives a finite contribution say $C_{1}$ ), we are left with

$$
\begin{aligned}
& \left|\Gamma_{4 c, \gamma_{1} \neq 0,(\mathrm{III})}^{(1)}\left[\bar{\varphi}_{1}, \varphi_{2}, \bar{\varphi}_{3}, \varphi_{4}\right]\right| \\
& \leq C_{1}+\frac{\lambda^{2}}{4 \theta^{2}} \sum_{\gamma_{2}^{*}}\left|\left\langle\varphi_{3}, \varphi_{4} U_{i \gamma_{2}^{*}}\right\rangle_{B}\right| \sum_{\gamma_{1} \neq 0}\left|\left\langle\varphi_{1}, \varphi_{2} U_{\gamma_{1}}\right\rangle_{B}\right| \\
& \cdot \int_{0}^{1} d \beta_{1} d \beta_{2} \frac{\sup _{\gamma_{2}^{*}}\left\{e^{-\frac{\omega}{2} \frac{e^{-\beta_{1} \omega}+e^{-\beta_{2} \omega}}{1-e^{-\left(\beta_{1}+\beta_{2}\right) \omega}}\left|\gamma_{1}+i \gamma_{2}^{*}\right|^{2}}\right\}}{\left(1-e^{-\left(\beta_{1}+\beta_{2}\right) \omega}\right)^{2}} \\
& \leq C_{1}+C_{2} \sum_{\gamma_{1} \neq 0}\left|\left\langle\varphi_{1}, \varphi_{2} U_{\gamma_{1}}\right\rangle_{B}\right| \int_{0}^{1} d \beta_{1} d \beta_{2} \frac{e^{-\frac{\omega}{2} \frac{e^{-\beta_{1} \omega}+e^{-\beta_{2} \omega}}{1-e^{-\left(\beta_{1}+\beta_{2}\right) \omega}} \inf _{\gamma_{2}^{*}}\left|\gamma_{1}+i \gamma_{2}^{*}\right|^{2}}}{\left(1-e^{-\left(\beta_{1}+\beta_{2}\right) \omega}\right)^{2}} \\
& \leq C_{1}+C_{2} \sum_{\gamma_{1} \neq 0}\left|\left\langle\varphi_{1}, \varphi_{2} U_{\gamma_{1}}\right\rangle_{B}\right| \int_{0}^{1} d \beta_{1} d \beta_{2} \frac{e^{-\frac{C \omega}{2} \frac{e^{-\beta_{1} \omega}}{1-e^{-\left(\beta_{1}+\beta_{2}\right) \omega}\left|\gamma_{1}\right|^{-2(4+\delta)}}}}{\left(1-e^{-\left(\beta_{1}+\beta_{2}\right) \omega}\right)^{2}} \\
& \leq C_{1}+\sum_{\gamma_{1} \neq 0}\left|\left\langle\varphi_{1}, \varphi_{2} U_{\gamma_{1}}\right\rangle_{B}\right|\left(C_{3}+C_{4} \ln \left|\gamma_{1}\right|\right),
\end{aligned}
$$

which is finite because $\left\langle\varphi_{i}, \varphi_{j} U_{\gamma}\right\rangle_{B}$ is a Schwartz sequence.

It should be clear that the same conclusion holds with the weak Diophantine condition (4.22) instead. The reason why this works is the logarithmic divergence of this graph. It is unclear to us if this condition will suffice at higher loop order. There is a general argument [6] that oriented just renormalisable $\phi^{4}$-models do not have quadratically divergent non-planar graphs. If this argument applies to our case, the weak Diophantine condition might suffice in general.

The second non-planar graph we have to compute is

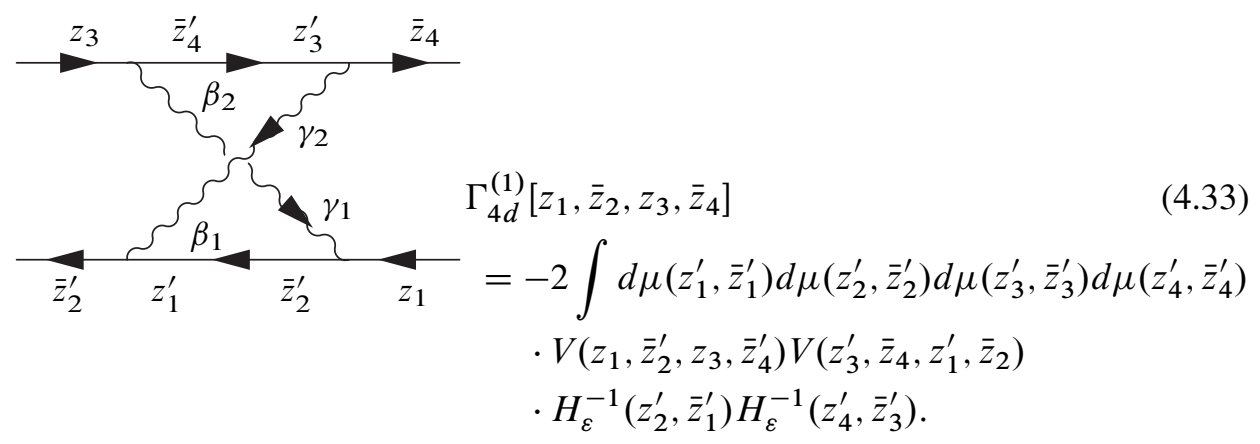


We have

$$
\begin{aligned}
& \Gamma_{4 d}^{(1)}\left[z_{1}, \bar{z}_{2}, z_{3}, \bar{z}_{4}\right] \\
& =-\frac{\lambda^{2}}{8} \sum_{\gamma_{1}, \gamma_{2}} \int_{\varepsilon}^{\infty} d \beta_{1} d \beta_{2} e^{-\frac{\mu_{0}^{2}}{2}\left(\beta_{1}+\beta_{2}\right)} e^{-\omega\left(\left|\gamma_{1}\right|^{2}+\left|\gamma_{2}\right|^{2}\right)} e^{\omega\left(\bar{z}_{2} \gamma_{2}+\left(\bar{z}_{2}-\bar{\gamma}_{2}\right) e^{-\beta_{1} \omega} \gamma_{1}\right)} \\
& \quad \cdot e^{\omega\left(\left(\bar{z}_{2}-\bar{\gamma}_{2}\right) e^{-\beta_{1} \omega} z_{1}-\bar{\gamma}_{1} z_{1}-\bar{z}_{4} \gamma_{2}-\left(\bar{z}_{4}+\bar{\gamma}_{2}\right) e^{-\beta_{2} \omega} \gamma_{1}+\left(\bar{z}_{4}+\bar{\gamma}_{2}\right) e^{-\beta_{2} \omega} z_{3}+\bar{\gamma}_{1} z_{3}\right)} .
\end{aligned}
$$

We set $\gamma_{2}=-\gamma_{1}+\gamma$. The resulting Gaußian decay in $\gamma_{1}$ is suppressed for small $\beta_{i}$, which is best expressed by a Poisson re-summation in $\gamma_{1}$ :

$$
\begin{gathered}
\Gamma_{4 d}^{(1)}\left[z_{1}, \bar{z}_{2}, z_{3}, \bar{z}_{4}\right] \\
=-\frac{\lambda^{2}}{8 \theta^{2}} \sum_{\gamma_{1}^{*}, \gamma} \int_{\varepsilon}^{\infty} \frac{d \beta_{1} d \beta_{2} e^{-\frac{\mu_{0}^{2}}{2}\left(\beta_{1}+\beta_{2}\right)}}{\left(2-e^{-\beta_{1} \omega}-e^{-\beta_{2} \omega}\right)^{2}} \\
\cdot e^{\omega\left(\left(\bar{z}_{2}-\bar{z}_{4}\right) \gamma-|\gamma|^{2}+\left(\bar{z}_{2}-\bar{\gamma}\right) e^{-\beta_{1} \omega} z_{1}+\left(\bar{z}_{4}+\bar{\gamma}\right) e^{-\beta_{2} \omega} z_{3}\right)} \\
\cdot e^{\frac{\omega}{\left(2-e^{-\beta_{1} \omega}-e^{-\beta_{2} \omega}\right)}\left(\bar{\gamma}\left(1-e^{-\beta_{1} \omega}-e^{-\beta_{2} \omega}\right)-\bar{z}_{2}\left(1-e^{-\beta_{1} \omega}\right)+\right.} \\
=-\frac{\lambda^{2}}{8 \theta^{2}} \sum_{\gamma_{1}^{*}, \gamma} \int_{\varepsilon}^{\infty} \frac{d \beta_{1} d \beta_{2} e^{-\frac{\mu_{0}^{2}}{2}\left(\beta_{1}+\beta_{2}\right)}}{\left(2-e^{-\beta_{1} \omega}-e^{-\beta_{2} \omega}\right)^{2}} e^{\omega\left(\left(\bar{z}_{2}-\bar{z}_{4}\right) \gamma+\bar{\gamma}\left(z_{3}-z_{1}\right)+\bar{z}_{2} z_{1}+\bar{z}_{4} z_{3}-\bar{\gamma} i \gamma_{1}^{*}\right)} \\
\cdot e^{-\frac{\left.\omega \bar{z}_{4}\left(1-e^{-\beta_{2} \omega}\right)-i \bar{\gamma}_{1}^{*}\right)\left(\gamma-\left(1-e^{-\beta_{1} \omega}\right) z_{1}+\left(1-e^{-\beta_{2} \omega}\right) z_{3}-i \gamma_{1}^{*}\right)}{\left(2-e^{-\beta_{1} \omega}-e^{-\beta_{2} \omega}\right)}\left(\left|\gamma-i \gamma_{1}^{*}\right|^{2}+\left(\overline{\left.\gamma-i \gamma_{1}^{*}\right)}\right)\left(\left(1-e^{-\beta_{2} \omega}\right) z_{3}-\right.\right.} \\
\cdot e^{-\frac{\omega\left(1-e^{-\beta_{1} \omega}\right)\left(1-e^{-\beta_{2} \omega}\right)}{\left(2-e^{-\beta_{1} \omega}-e^{-\beta_{2} \omega}\right)}\left(\bar{z}_{2}+\bar{z}_{4}\right)\left(z_{1}+z_{3}\right)} .
\end{gathered}
$$

When $\theta$ is irrational and satisfies a Diophantine condition (Definition 4.4) to control the sums $\gamma, \gamma_{1}^{*} \neq 0$, we see that only the mode $\gamma=0, \gamma_{1}^{*}=0$ will contribute to the diverging part. Adding external fields and integrating over the $z_{i}$-positions with the help of the reproducing kernels, we obtain in this case

$$
\begin{aligned}
& \Gamma_{4 d, \gamma=\gamma_{1}^{*}=0}^{(1)}\left[\bar{\varphi}_{1}, \varphi_{2}, \bar{\varphi}_{3}, \varphi_{4}\right] \\
&=-\frac{\lambda^{2}}{8 \theta^{2}} \int d \mu\left(z_{1}, \bar{z}_{1}\right) d \mu\left(z_{3}, \bar{z}_{3}\right) \int_{\varepsilon}^{\infty} \frac{d \beta_{1} d \beta_{2} e^{-\frac{\mu_{0}^{2}}{2}\left(\beta_{1}+\beta_{2}\right)}}{\left(2-e^{-\beta_{1} \omega}-e^{-\beta_{2} \omega}\right)^{2}} \\
& \bar{\varphi}_{1}\left(\bar{z}_{1}\right) \varphi_{2}\left(z_{1}-\frac{\omega\left(1-e^{-\beta_{1} \omega}\right)\left(1-e^{-\beta_{2} \omega}\right)}{\left(2-e^{-\beta_{1} \omega}-e^{-\beta_{2} \omega}\right)}\left(z_{1}+z_{3}\right)\right) \\
& \bar{\varphi}_{3}\left(\bar{z}_{3}\right) \varphi_{4}\left(z_{3}-\frac{\omega\left(1-e^{-\beta_{1} \omega}\right)\left(1-e^{-\beta_{2} \omega}\right)}{\left(2-e^{-\beta_{1} \omega}-e^{-\beta_{2} \omega}\right)}\left(z_{1}+z_{3}\right)\right) .
\end{aligned}
$$


Now, using Taylor's theorem in the form $\varphi(z+y)=\varphi(z)+y \int_{0}^{1} d \xi(\partial \varphi)(z+\xi y)$, it is easy to see that the derivatives of $\varphi$ lead to a convergent $\beta$-integral. Therefore the divergent part is given by

$$
\Gamma_{4 d, \mathrm{div}}^{(1)}\left[\bar{\varphi}_{1}, \varphi_{2}, \bar{\varphi}_{3}, \varphi_{4}\right]=-\frac{\lambda^{2}}{8 \theta^{2}}\left\langle\varphi_{1}, \varphi_{2}\right\rangle_{B}\left\langle\varphi_{3}, \varphi_{3}\right\rangle_{B} \int_{\varepsilon}^{\infty} \frac{d \beta_{1} d \beta_{2} e^{-\frac{\mu_{0}^{2}}{2}\left(\beta_{1}+\beta_{2}\right)}}{\left(2-e^{-\beta_{1} \omega}-e^{-\beta_{2} \omega}\right)^{2}}
$$

Again this divergence is quite problematic at this stage since it corresponds to a counterterm of the form (4.30), which was not present in the classical action we have chosen (2.33).

For $\theta \in \mathbb{N}$, the divergent part of the graph (4.33) is given by the mode $\gamma_{1}^{*}=-i \gamma$. Using again Taylor's theorem we obtain

$$
\Gamma_{4 d, \theta \in \mathbb{N}, \operatorname{div}}^{(1)}\left[z_{1}, \bar{z}_{2}, z_{3}, \bar{z}_{4}\right]=-\frac{\lambda}{4 \theta^{2}} V\left(z_{1}, \bar{z}_{2}, z_{3}, \bar{z}_{4}\right) \int_{\varepsilon}^{\infty} \frac{d \beta_{1} d \beta_{2} e^{-\frac{\mu_{0}^{2}}{2}\left(\beta_{1}+\beta_{2}\right)}}{\left(2-e^{-\beta_{1} \omega}-e^{-\beta_{2} \omega}\right)^{2}} .
$$

In contrast to the irrational case, this is now a local counterterm. For $\theta$ rational, the same discussion than in the previous graph applies and we are left with a divergence of the form (4.28).

It remains to show that in the irrational Diophantian case, the remaining sum over $\left(\gamma, \gamma_{1}^{*}\right) \neq(0,0)$ leads to a convergent integral. With the help of reproducing kernels we find for (4.34)

$\Gamma_{4 d, \theta \notin \mathbb{Q}, \text { conv }}^{(1)}\left[\bar{\varphi}_{1}, \varphi_{2}, \bar{\varphi}_{3}, \varphi_{4}\right]$

$$
\begin{aligned}
& =-\frac{\lambda^{2}}{8 \theta^{2}} \sum_{\left(\gamma_{1}^{*}, \gamma\right) \neq(0,0)} \int d \mu\left(z_{1}, \bar{z}_{1}\right) d \mu\left(z_{3}, \bar{z}_{3}\right) \int_{\varepsilon}^{\infty} \frac{d \beta_{1} d \beta_{2} e^{-\frac{\mu_{0}^{2}}{2}\left(\beta_{1}+\beta_{2}\right)}}{\left(2-e^{-\beta_{1} \omega}-e^{-\beta_{2} \omega}\right)^{2}} \\
& \cdot \bar{\varphi}_{1}\left(\bar{z}_{1}\right) \varphi_{2}\left(z_{1}+\gamma-\frac{\left(1-e^{-\beta_{1} \omega}\right)}{\left(2-e^{-\beta_{1} \omega}-e^{-\beta_{2} \omega}\right)}\left(\gamma-i \gamma_{1}^{*}\right)-\frac{\left(1-e^{-\beta_{1} \omega}\right)\left(1-e^{-\beta_{2} \omega}\right)}{\left(2-e^{-\beta_{1} \omega}-e^{-\beta_{2} \omega}\right)}\left(z_{1}+z_{3}\right)\right) \\
& \cdot \bar{\varphi}_{3}\left(\bar{z}_{3}\right) \varphi_{4}\left(z_{3}-\gamma+\frac{\left(1-e^{-\beta_{2} \omega}\right)}{\left(2-e^{-\beta_{1} \omega}-e^{-\beta_{2} \omega}\right)}\left(\gamma-i \gamma_{1}^{*}\right)-\frac{\left(1-e^{-\beta_{1} \omega}\right)\left(1-e^{-\beta_{2} \omega}\right)}{\left(2-e^{-\beta_{1} \omega}-e^{-\beta_{2} \omega}\right)}\left(z_{1}+z_{3}\right)\right) \\
& \cdot e^{\omega\left(\bar{\gamma}\left(z_{3}-z_{1}\right)-\bar{\gamma} i \gamma_{1}^{*}\right)} e^{-\frac{\omega}{\left(2-e^{-\beta_{1} \omega}-e^{-\beta_{2} \omega}\right)}\left(\left|\gamma-i \gamma_{1}^{*}\right|^{2}+\left(\frac{\gamma-i \gamma_{1}^{*}}{2}\right)\left(\left(1-e^{-\beta_{2} \omega}\right) z_{3}-\left(1-e^{-\beta_{1} \omega}\right) z_{1}\right)\right)} .
\end{aligned}
$$

We re-express $\gamma, \gamma_{1}^{*}$-dependence of the field positions in terms of the unitaries $U$ :

$$
\begin{aligned}
& \Gamma_{4 d, \theta \notin \mathbb{Q}, \text { conv }}^{(1)}\left[\bar{\varphi}_{1}, \varphi_{2}, \bar{\varphi}_{3}, \varphi_{4}\right] \\
& \quad=-\frac{\lambda^{2}}{8 \theta^{2}} \sum_{\left(\gamma_{1}^{*}, \gamma\right) \neq(0,0)} \int d \mu\left(z_{1}, \bar{z}_{1}\right) d \mu\left(z_{3}, \bar{z}_{3}\right) \int_{\varepsilon}^{\infty} \frac{d \beta_{1} d \beta_{2} e^{-\frac{\mu_{0}^{2}}{2}\left(\beta_{1}+\beta_{2}\right)}}{\left(2-e^{-\beta_{1} \omega}-e^{-\beta_{2} \omega}\right)^{2}}
\end{aligned}
$$




$$
\begin{aligned}
& \cdot \bar{\varphi}_{1}\left(\bar{z}_{1}\right)\left(\varphi_{2} U_{\gamma} U_{-\frac{\left(1-e^{-\beta_{1} \omega}\right)\left(\gamma-i \gamma_{1}^{*}\right)}{\left(2-e^{-\beta_{1} \omega}-e^{-\beta_{2} \omega}\right)}}\right)\left(z_{1}-\frac{\left(1-e^{-\beta_{1} \omega}\right)\left(1-e^{-\beta_{2} \omega}\right)}{\left(2-e^{-\beta_{1} \omega}-e^{-\beta_{2} \omega}\right)}\left(z_{1}+z_{3}\right)\right) \\
& \cdot \bar{\varphi}_{3}\left(\bar{z}_{3}\right)\left(\varphi_{4} U_{-\gamma} U_{\frac{\left(1-e^{-\beta_{2} \omega}\right)\left(\gamma-i \gamma_{1}^{*}\right)}{\left(2-e^{-\beta_{1} \omega}-e^{-\beta_{2} \omega}\right)}}\right)\left(z_{3}-\frac{\left(1-e^{-\beta_{1} \omega}\right)\left(1-e^{-\beta_{2} \omega}\right)}{\left(2-e^{-\beta_{1} \omega}-e^{-\beta_{2} \omega}\right)}\left(z_{1}+z_{3}\right)\right) \\
& \cdot e^{-\frac{\omega}{\left(2-e^{-\beta_{1} \omega}-e^{-\beta_{2} \omega}\right)}\left|\gamma-i \gamma_{1}^{*}\right|^{2}\left(1-\frac{\left(1-e^{-\beta_{1} \omega}\right)^{2}+\left(1-e^{-\beta_{2} \omega}\right)^{2}}{2\left(2-e^{-\beta_{1} \omega}-e^{-\beta_{2} \omega}\right)}\right)} \\
& \cdot e^{-\frac{\omega\left(1-e^{-\beta_{1} \omega}\right)\left(1-e^{-\beta_{2} \omega}\right)\left(e^{-\beta_{1} \omega}-e^{-\beta_{2} \omega}\right)}{\left(2-e^{-\beta_{1} \omega}-e^{-\beta_{2} \omega}\right)^{2}}\left(\overline{\gamma-i \gamma_{1}^{*}}\right)\left(z_{1}+z_{3}\right)} .
\end{aligned}
$$

Now we absorb the last line in new unitaries, at the price of $\left(\gamma-i \gamma_{1}^{*}\right)$-dependent positions:

$$
\begin{aligned}
& \Gamma_{4 d, \theta \notin \mathbb{Q}, \mathrm{conv}}^{(1)}\left[\bar{\varphi}_{1}, \varphi_{2}, \bar{\varphi}_{3}, \varphi_{4}\right] \\
& =-\frac{\lambda^{2}}{8 \theta^{2}} \sum_{\left(\gamma_{1}^{*}, \gamma\right) \neq(0,0)} \int d \mu\left(z_{1}, \bar{z}_{1}\right) d \mu\left(z_{3}, \bar{z}_{2}\right) \int_{\varepsilon}^{\infty} \frac{d \beta_{1} d \beta_{2} e^{-\frac{\mu_{0}^{2}}{2}\left(\beta_{1}+\beta_{2}\right)}}{\left(2-e^{-\beta_{1} \omega}-e^{-\beta_{2} \omega}\right)^{2}} \\
& \text { - } \bar{\varphi}_{1}\left(\bar{z}_{1}\right)\left(\varphi_{2} U_{-\gamma} U_{f_{1}\left(\beta_{1}, \beta_{2}\right)\left(\gamma-i \gamma_{1}^{*}\right)} U_{f_{2}\left(\beta_{1}, \beta_{2}\right)\left(\gamma-i \gamma_{1}^{*}\right)}\right)\left(\tilde{z}_{1}\right) \\
& \cdot \bar{\varphi}_{3}\left(\bar{z}_{3}\right)\left(\varphi_{4} U_{\gamma} U_{-f_{1}\left(\beta_{2}, \beta_{1}\right)\left(\gamma-i \gamma_{1}^{*}\right)} U_{f_{2}\left(\beta_{1}, \beta_{2}\right)\left(\gamma-i \gamma_{1}^{*}\right)}\right)\left(\tilde{z}_{3}\right) \\
& \cdot e^{-\frac{\omega\left|\gamma-i \gamma_{1}^{*}\right|^{2}}{\left(2-e^{-\beta_{1} \omega}-e^{-\beta_{2} \omega}\right)}\left(1-\frac{\left(1-e^{-\beta_{1} \omega}\right)^{2}+\left(1-e^{-\beta_{2} \omega}\right)^{2}}{2\left(2-e^{-\beta_{1} \omega}-e^{-\beta_{2} \omega}\right)}+\right.} \\
& \left.+\frac{\left(1-e^{-\beta_{1} \omega}\right)^{2}\left(1-e^{-\beta_{2} \omega}\right)^{2}\left(e^{-\beta_{1} \omega}-e^{-\beta_{2} \omega}\right)^{2}}{4\left(2-e^{-\beta_{1} \omega}-e^{-\beta_{2} \omega}\right)\left(e^{-\beta_{2} \omega}\left(1-e^{-\beta_{1} \omega}\right)+e^{-\beta_{1} \omega}\left(1-e^{-\beta_{2} \omega}\right)\right)^{2}}\right),
\end{aligned}
$$

where we have set

$$
\begin{aligned}
f_{1}\left(\beta_{1}, \beta_{2}\right) & =\frac{\left(1-e^{-\beta_{1} \omega}\right)}{\left(2-e^{-\beta_{1} \omega}-e^{-\beta_{2} \omega}\right)}, \\
f_{2}\left(\beta_{1}, \beta_{2}\right) & =\frac{\left(1-e^{-\beta_{1} \omega}\right)\left(1-e^{-\beta_{2} \omega}\right)\left(e^{-\beta_{1} \omega}-e^{-\beta_{2} \omega}\right)}{2\left(2-e^{-\beta_{1} \omega}-e^{-\beta_{2} \omega}\right)\left(e^{-\beta_{2} \omega}\left(1-e^{-\beta_{1} \omega}\right)+e^{-\beta_{1} \omega}\left(1-e^{-\beta_{2} \omega}\right)\right)}, \\
f_{3}\left(\beta_{1}, \beta_{2}\right) & =\frac{\left(1-e^{-\beta_{1} \omega}\right)\left(1-e^{-\beta_{2} \omega}\right)}{\left(2-e^{-\beta_{1} \omega}-e^{-\beta_{2} \omega}\right)} . \\
\tilde{z}_{1} & =z_{1}-f_{3}\left(\beta_{1}, \beta_{2}\right)\left(z_{1}+z_{3}\right)-f_{2}\left(\beta_{1}, \beta_{2}\right)\left(\gamma-i \gamma_{1}^{*}\right) \\
\tilde{z}_{3} & =z_{3}-f_{3}\left(\beta_{1}, \beta_{2}\right)\left(z_{1}+z_{3}\right)-f_{2}\left(\beta_{1}, \beta_{2}\right)\left(\gamma-i \gamma_{1}^{*}\right)
\end{aligned}
$$

Since

$$
1-\frac{\left(1-e^{-\beta_{1} \omega}\right)^{2}+\left(1-e^{-\beta_{2} \omega}\right)^{2}}{2\left(2-e^{-\beta_{1} \omega}-e^{-\beta_{2} \omega}\right)} \geq \frac{1}{2},
$$


we obtain

$$
\begin{aligned}
& \left|\Gamma_{4 d, \theta \notin \mathbb{Q}, \mathrm{conv}}^{(1)}\left[\bar{\varphi}_{1}, \varphi_{2}, \bar{\varphi}_{3}, \varphi_{4}\right]\right| \\
& \leq \frac{\lambda^{2}}{8 \theta^{2}} \sum_{\left(\gamma_{1}^{*}, \gamma\right) \neq(0,0)} \int_{\varepsilon}^{\infty} \frac{d \beta_{1} d \beta_{2} e^{-\frac{\mu_{0}^{2}}{2}\left(\beta_{1}+\beta_{2}\right)}}{\left(2-e^{-\beta_{1} \omega}-e^{-\beta_{2} \omega}\right)^{2}} e^{-\frac{\omega\left|\gamma-i \gamma_{1}^{*}\right|^{2}}{2\left(2-e^{-\beta_{1} \omega}-e^{-\beta_{2} \omega}\right)}} \\
& \cdot \mid \int d \mu\left(z_{1}, \bar{z}_{1}\right) d \mu\left(z_{3}, \bar{z}_{3}\right) \bar{\varphi}_{1}\left(\bar{z}_{1}\right) \bar{\varphi}_{3}\left(\bar{z}_{3}\right) \\
& \text { - }\left(\varphi_{2} U_{-\gamma} U_{f_{1}\left(\beta_{1}, \beta_{2}\right)\left(\gamma-i \gamma_{1}^{*}\right)} U_{f_{2}\left(\beta_{1}, \beta_{2}\right)\left(\gamma-i \gamma_{1}^{*}\right)}\right)\left(\tilde{z}_{1}\right) \\
& \cdot\left(\varphi_{4} U_{\gamma} U_{-f_{1}\left(\beta_{2}, \beta_{1}\right)\left(\gamma-i \gamma_{1}^{*}\right)} U_{f_{2}\left(\beta_{1}, \beta_{2}\right)\left(\gamma-i \gamma_{1}^{*}\right)}\right)\left(\tilde{z}_{3}\right) \mid .
\end{aligned}
$$

The next step consists in disregarding the term $f_{3}\left(\beta_{1}, \beta_{2}\right)\left(z_{1}+z_{3}\right)+f_{2}\left(\beta_{1}, \beta_{2}\right)$ $\left(\gamma-i \gamma_{1}^{*}\right)$ in the argument of $\varphi_{2}, \varphi_{4}$. Indeed, any $\phi \in \mathcal{E}_{B}\left(\mathbb{C}^{2}\right)=B \delta\left(\mathbb{R}^{2}\right)$ can be written as $\phi(z)=\sum_{n} \phi_{n} z^{n}$, where $\left\{\phi_{n}\right\} \in \delta(\mathbb{N})$. This is true because any $f \in \delta\left(\mathbb{R}^{2}\right)$ can be expanded as a sum of the eigen-modes of the harmonic oscillator, with rapidly decreasing coefficients. So to apply that to $\varphi_{2} U_{-\gamma} U_{f_{1}\left(\beta_{1}, \beta_{2}\right)\left(\gamma-i \gamma_{1}^{*}\right)} U_{f_{2}\left(\beta_{1}, \beta_{2}\right)\left(\gamma-i \gamma_{1}^{*}\right)}$, it is enough to analyse the first term in the expansion of

$$
\left(z_{1}-f_{3}\left(\beta_{1}, \beta_{2}\right)\left(z_{1}+z_{3}\right)-f_{2}\left(\beta_{1}, \beta_{2}\right)\left(\gamma-i \gamma_{1}^{*}\right)\right)^{n},
$$

since they are all of the same weight because $f_{i}\left(\beta_{1}, \beta_{2}\right) \in L^{\infty}\left(\mathbb{R}^{+} \times \mathbb{R}^{+}\right), i=1,2,3$. Thus each term can be estimated along the same lines as the first one, with estimates uniform in $\beta_{i}, \gamma, \gamma_{1}^{*}$. Finally, the Schwartz coefficients will absorb the overall number of terms of the expansion in $z^{n}$. Thus, disregarding the integral from 1 to $+\infty$ which gives a finite contribution $C_{1}$, we are left with

$$
\begin{aligned}
&\left|\Gamma_{4 d, \theta \notin \mathbb{Q}, \text { conv }}^{(1)}\left[\bar{\varphi}_{1}, \varphi_{2}, \bar{\varphi}_{3}, \varphi_{4}\right]\right| \\
& \leq C_{1}+\frac{\lambda^{2}}{8 \theta^{2}} \sum_{\left(\gamma_{1}^{*}, \gamma\right) \neq(0,0)} \int_{\varepsilon} \frac{d \beta_{1} d \beta_{2} e^{-\frac{\mu_{0}^{2}}{2}\left(\beta_{1}+\beta_{2}\right)}}{\left(2-e^{-\beta_{1} \omega}-e^{-\beta_{2} \omega}\right)^{2}} e^{-\frac{\omega\left|\gamma-i \gamma_{1}^{*}\right|^{2}}{2\left(2-e^{-\beta_{1} \omega}-e^{-\beta_{2} \omega}\right)}} \\
& \cdot \mid\left\langle\varphi_{1}, \varphi_{2} U_{-\gamma+\left(f_{1}\left(\beta_{1}, \beta_{2}\right)+f_{2}\left(\beta_{1}, \beta_{2}\right)\left(\gamma-i \gamma_{1}^{*}\right)\right\rangle_{B}}\right. \\
& \cdot\left\langle\varphi_{3}, \varphi_{4} U_{\gamma-\left(f_{1}\left(\beta_{2}, \beta_{1}\right)-f_{2}\left(\beta_{1}, \beta_{2}\right)\left(\gamma-i \gamma_{1}^{*}\right)\right\rangle_{B} \mid .}\right.
\end{aligned}
$$

Since $\left\langle\phi, \chi U_{\eta}\right\rangle_{B}$ belongs to $\delta\left(\mathbb{R}^{4}\right)$ as a function of $\eta \in \mathbb{C}^{2} \simeq \mathbb{R}^{4}$, and since $f_{2} \rightarrow 0$, $\beta_{1}, \beta_{2} \rightarrow 0$ and $f_{1} \rightarrow 1, \beta_{1}, \beta_{2} \rightarrow 0, \beta_{1}=\beta_{2}$ (which is the only important case to treat), we deduce that

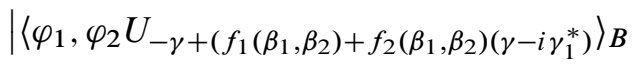

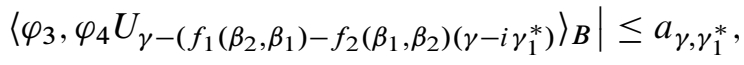


uniformly in $\beta_{1}, \beta_{2}$ and where $0 \leq\left\{a_{\gamma, \gamma_{1}^{*}}\right\} \in \S\left(\mathbb{Z}^{8}\right)$. Finally, we get

$$
\begin{aligned}
& \left|\Gamma_{4 d, \theta \notin \mathbb{Q}, \mathrm{conv}}^{(1)}\left[\bar{\varphi}_{1}, \varphi_{2}, \bar{\varphi}_{3}, \varphi_{4}\right]\right| \\
& \quad \leq C_{1}+\sum_{\left(\gamma_{1}^{*}, \gamma\right) \neq(0,0)} a_{\gamma, \gamma_{1}^{*}} \int_{\varepsilon}^{1} \frac{d \beta_{1} d \beta_{2} e^{-\frac{\mu_{0}^{2}}{2}\left(\beta_{1}+\beta_{2}\right)}}{\left(2-e^{-\beta_{1} \omega}-e^{-\beta_{2} \omega}\right)^{2}} e^{-\frac{\omega\left|\gamma-i \gamma_{1}^{*}\right|^{2}}{2\left(2-e^{-\beta_{1} \omega}-e^{-\beta_{2} \omega}\right)}} .
\end{aligned}
$$

Thus the estimate (4.32) of the previous diagram applies as well and shows that the limit $\epsilon \rightarrow 0$ is finite.

Gathering all results we have proven:

Proposition 4.5. When $\theta \in \mathbb{Q}$, the divergent part of the 4-point non-planar 1 PI Green function associated with the action (2.33), in its 1-loop approximation, is given by

$$
\begin{aligned}
& \Gamma_{4, \text { non-planar,div }}^{(1)}\left[M_{1}^{\dagger}, M_{2}, M_{3}^{\dagger}, M_{4}\right] \\
& \quad=-\frac{3 \lambda^{2}}{8 \omega^{2} \theta^{2}} \int_{0}^{\frac{1}{q}} d x \int_{0}^{\frac{1}{q}} d y \operatorname{Tr}\left[M_{1}^{\dagger} M_{2}\right](x, y) \operatorname{Tr}\left[M_{3}^{\dagger} M_{4}\right](x, y) \ln \frac{1}{\epsilon},
\end{aligned}
$$

and when $\theta \in \mathbb{R} \backslash \mathbb{Q}$ and satisfies the weak Diophantine condition (4.22), it is given by

$$
\Gamma_{4, \text { non-planar,div }}^{(1)}\left[\bar{\varphi}_{1}, \varphi_{2}, \bar{\varphi}_{3}, \varphi_{4}\right]=-\frac{3 \lambda^{2}}{8 \omega^{2} \theta^{2}} \operatorname{Tr}_{\mathcal{A}_{\theta}}\left[\left(\varphi_{1}, \varphi_{2}\right)_{\mathcal{A}_{\theta}}\right] \operatorname{Tr}_{\mathcal{A}_{\theta}}\left[\left(\varphi_{3}, \varphi_{4}\right)_{\mathcal{A}_{\theta}}\right] \ln \frac{1}{\epsilon} .
$$

In the irrational case this analysis leads to the introduction of a non-local term in the action (2.33) associated to the new divergence and with a second coupling constant:

$$
\begin{aligned}
S[\phi, \bar{\phi}]= & g^{\mu \nu} \operatorname{Tr}_{\mathcal{A}_{\Theta}}\left[\left(\nabla_{\mu} \phi, \nabla_{\nu} \phi\right)_{\mathcal{A}_{\Theta}}\right]+\mu_{0}^{2} \operatorname{Tr}_{\mathcal{A}_{\Theta}}\left[(\phi, \phi)_{\mathcal{A}_{\Theta}}\right] \\
& +\frac{\lambda}{2} \operatorname{Tr}_{\mathcal{A}_{\Theta}}\left[(\phi, \phi)_{\mathcal{A}_{\Theta}}^{2}\right]+\frac{\lambda^{\prime}}{2}\left[\operatorname{Tr}_{\mathcal{A}_{\theta}}\left[(\phi, \phi)_{\mathcal{A}_{\theta}}\right]\right]^{2} .
\end{aligned}
$$

Such a term also appeared in [1].

To show that our theory is now reasonably well defined, we have to show that the divergences coming from loop diagrams constructed with the new vertex (and also with mixed vertices) can be absorbed by a redefinition of $\mu_{0}, \lambda, \lambda^{\prime}$. This is shown to hold in the 1-loop approximation in the next section. 


\section{Mixed diagrams}

We symbolise the new vertex by a dotted line:

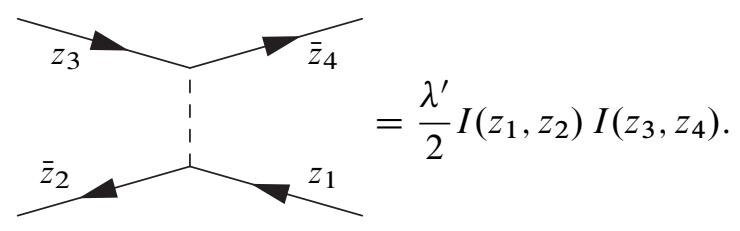

This interaction $\left(\operatorname{Tr}_{\mathcal{A}_{\theta}}\left[(\phi, \phi)_{\mathcal{A}_{\theta}}\right]\right)^{2}$ can also be written using the HubbardStratonovitch transform as

$$
e^{-\frac{\lambda^{\prime}}{2}\left(\operatorname{Tr}_{\mathcal{A}_{\theta}}\left[(\phi, \phi)_{\mathcal{A}_{\theta}}\right]\right)^{2}}=\sqrt{\frac{\lambda^{\prime}}{2 \pi}} \int_{\mathbb{R}} d a e^{-\frac{\lambda^{\prime}}{2} a^{2}-i \lambda^{\prime} a \operatorname{Tr}_{\mathcal{A}_{\theta}}\left[(\phi, \phi)_{\mathcal{A}_{\theta}}\right]}
$$

where we integrate over a real number $a$. Equivalently, it can also be obtained by introducing a different coupling for the constant mode of the field $A$ appearing in the Hubbard-Stratonovitch transform.

5.1. 2-point sector. The first 2-point graph with the new vertex is thus

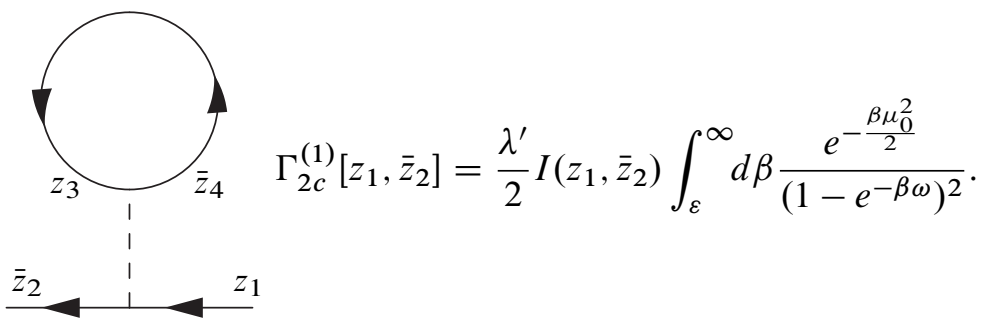

Its value is obtained from (4.5) by putting $\gamma \mapsto 0$ and $\lambda \mapsto \lambda^{\prime}$. This coincides exactly with the previous divergence.

The second contribution to the 1-loop 2-point function is the graph

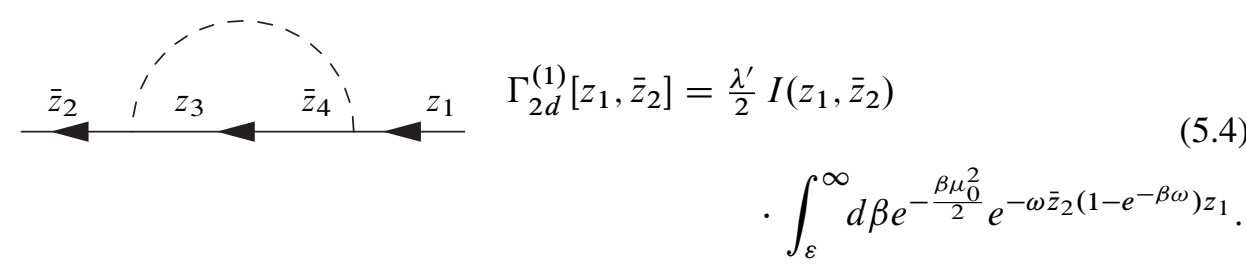

Again this is immediately obtained from (4.10). After insertion of external fields, the integral is obviously finite. 
5.2. 4-point sector: planar graphs. To each of the previous graphs there correspond three different new graphs. The mixed analogue of (4.13) is

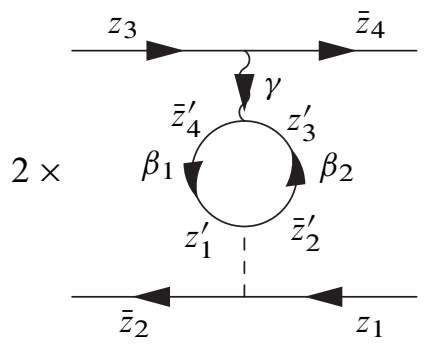

$$
\begin{aligned}
\Gamma_{4 e}^{(1)}\left[z_{1}, \bar{z}_{2}, z_{3}, \bar{z}_{4}\right]=- & \frac{\lambda \lambda^{\prime}}{4} \sum_{\gamma} I\left(z_{1}, \bar{z}_{2}\right) I\left(z_{3}, \bar{z}_{4}\right) \\
& \cdot \int_{\varepsilon}^{\infty} \frac{d \beta_{1} d \beta_{2} e^{-\frac{\mu_{0}^{2}}{2}\left(\beta_{1}+\beta_{2}\right)}}{\left(1-e^{-\left(\beta_{1}+\beta_{2}\right) \omega}\right)^{2}} \\
& \cdot e^{-\omega\left(\bar{z}_{4} \gamma-\bar{\gamma} z_{3}+\frac{|\gamma|^{2}}{1-e^{-\left(\beta_{1}+\beta_{2}\right) \omega}}\right)} .
\end{aligned}
$$

The graph where the new vertex is above has the same value so that we count this graphs twice. Its value is obtained from (4.14) by setting $\gamma_{1}=0$ and $\gamma_{2}=\gamma$. Obviously, only the mode $\gamma=0$ produces a divergence:

$$
\Gamma_{4 e, \mathrm{div}}^{(1)}\left[z_{1}, \bar{z}_{2}, z_{3}, \bar{z}_{4}\right]=-\frac{\lambda \lambda^{\prime}}{4} I\left(z_{1}, \bar{z}_{2}\right) I\left(z_{3}, \bar{z}_{4}\right) \int_{\varepsilon}^{\infty} \frac{d \beta_{1} d \beta_{2} e^{-\frac{\mu_{0}^{2}}{2}\left(\beta_{1}+\beta_{2}\right)}}{\left(1-e^{-\left(\beta_{1}+\beta_{2}\right) \omega}\right)^{2}} .
$$

There is also the analogue of (4.13) where both vertices are new ones:

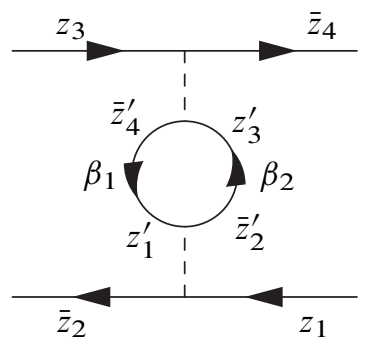

$$
\begin{aligned}
\Gamma_{4 f}^{(1)}\left[z_{1}, \bar{z}_{2}, z_{3}, \bar{z}_{4}\right]=- & \frac{\lambda^{\prime 2}}{8} I\left(z_{1}, z_{2}\right) I\left(z_{3}, z_{4}\right) \\
& \int_{\varepsilon}^{\infty} \frac{d \beta_{1} d \beta_{2} e^{-\frac{\mu_{0}^{2}}{2}\left(\beta_{1}+\beta_{2}\right)}}{\left(1-e^{-\left(\beta_{1}+\beta_{2}\right) \omega}\right)^{2}} .
\end{aligned}
$$

This gives directly the divergent part.

All mixed analogues of the graph (4.17) are finite because there is no loop sum- 
mation:

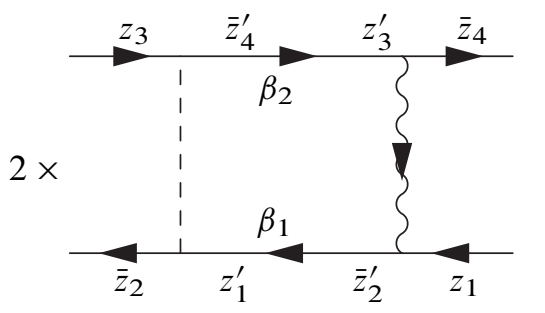

$$
\begin{aligned}
\Gamma_{4 g}^{(1)}\left[z_{1}, \bar{z}_{2}, z_{3}, \bar{z}_{4}\right] & \\
=- & \frac{\lambda \lambda^{\prime}}{4} \sum_{\gamma} \int_{\varepsilon}^{\infty} d \beta_{1} d \beta_{2} e^{-\frac{\mu_{0}^{2}}{2}\left(\beta_{1}+\beta_{2}\right)} \\
& \cdot e^{\omega\left(-\left|\gamma_{1}\right|^{2}-\bar{\gamma} z_{1}-\bar{z}_{4} \gamma\right)} \\
& \cdot e^{\omega\left(\bar{z}_{2} e^{-\beta_{1} \omega} \gamma+\bar{z}_{2} e^{-\beta_{1} \omega} z_{1}\right)} \\
& \cdot e^{\omega\left(\bar{z}_{4} e^{-\beta_{2} \omega} z_{3}+\bar{\gamma} e^{-\beta_{2} \omega} z_{3}\right)}
\end{aligned}
$$

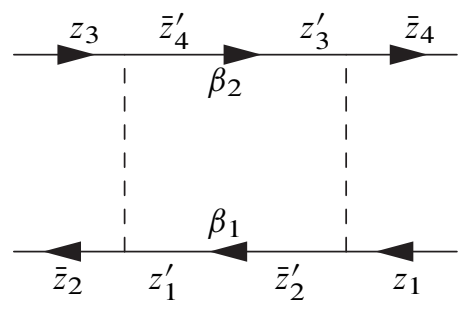

$$
\begin{aligned}
\Gamma_{4 h}^{(1)}\left[z_{1}, \bar{z}_{2}, z_{3}, \bar{z}_{4}\right] & \\
=- & \frac{\lambda^{\prime 2}}{8} \int_{\varepsilon}^{\infty} d \beta_{1} d \beta_{2} e^{-\frac{\mu_{0}^{2}}{2}\left(\beta_{1}+\beta_{2}\right)} \\
& \cdot e^{\omega\left(\bar{z}_{2} e^{-\beta_{1} \omega} z_{1}+\bar{z}_{4} e^{-\beta_{2} \omega} z_{3}\right)} .
\end{aligned}
$$

5.3. 4-point sector: non-planar graphs. The analogues of the first non-planar graph (4.23) have one possibility where a loop summation remains. Writing directly the result of the Poisson re-summation, we have

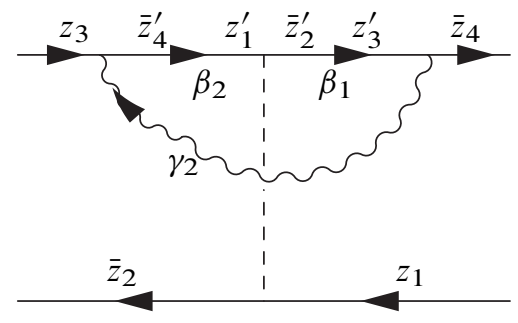

$$
\begin{aligned}
& \Gamma_{4 i}^{(1)}\left[z_{1}, \bar{z}_{2}, z_{3}, \bar{z}_{4}\right] \\
&=- \frac{\lambda \lambda^{\prime}}{4 \theta^{2}} \sum_{\gamma_{2}^{*}} e^{\omega\left(\bar{z}_{2} z_{1}+\bar{z}_{4} z_{3}+i \bar{\gamma}_{2}^{*} z_{3}+\bar{z}_{4} i \gamma_{2}^{*}\right)} \\
& \cdot \int_{\varepsilon}^{\infty} d \beta_{1} d \beta_{2} \frac{e^{-\frac{\mu_{0}^{2}}{2}\left(\beta_{1}+\beta_{2}\right)}}{\left(1-e^{-\left(\beta_{1}+\beta_{2}\right) \omega}\right)^{2}} \\
& \cdot e^{-\frac{\omega}{1-e^{-\left(\beta_{1}+\beta_{2}\right) \omega}\left|\gamma_{2}^{*}\right|^{2}}} .
\end{aligned}
$$


Only the mode $\gamma_{2}^{*}=0$ contributes to the divergence:

$$
\Gamma_{4 i, \text { div }}^{(1)}\left[z_{1}, \bar{z}_{2}, z_{3}, \bar{z}_{4}\right]=-\frac{\lambda \lambda^{\prime}}{4 \theta^{2}} I\left(z_{1}, \bar{z}_{2}\right) I\left(z_{3}, \bar{z}_{4}\right) \int_{\varepsilon}^{\infty} d \beta_{1} d \beta_{2} \frac{e^{-\frac{\mu_{0}^{2}}{2}\left(\beta_{1}+\beta_{2}\right)}}{\left(1-e^{-\left(\beta_{1}+\beta_{2}\right) \omega}\right)^{2}} .
$$

The other analogues of (4.23) are finite:

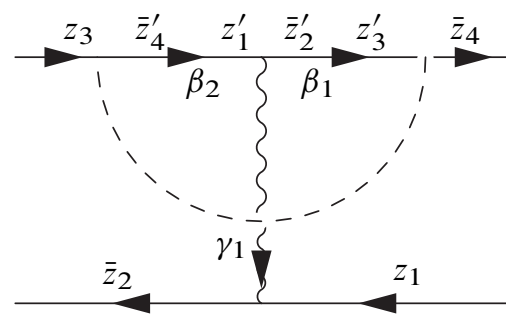

$$
\begin{aligned}
\Gamma_{4 j}^{(1)}\left[z_{1}, \bar{z}_{2}, z_{3}, \bar{z}_{4}\right] \\
=-\frac{\lambda \lambda^{\prime}}{4} \sum_{\gamma_{1}} \int_{\varepsilon}^{\infty} d \beta_{1} d \beta_{2} e^{-\frac{\mu_{0}^{2}}{2}\left(\beta_{1}+\beta_{2}\right)} \\
\cdot \cdot e^{\omega\left(-\left|\gamma_{1}\right|^{2}+\bar{z}_{2} \gamma_{1}+\bar{z}_{2} z_{1}-\bar{\gamma}_{1} z_{1}\right)} \\
\cdot e^{\omega\left(-\bar{z}_{4} e^{-\beta_{1} \omega} \gamma_{1}+\bar{z}_{4} e^{-\left(\beta_{1}+\beta_{2}\right) \omega} z_{3}+\bar{\gamma}_{1} e^{-\beta_{2} \omega} z_{3}\right)} .
\end{aligned}
$$

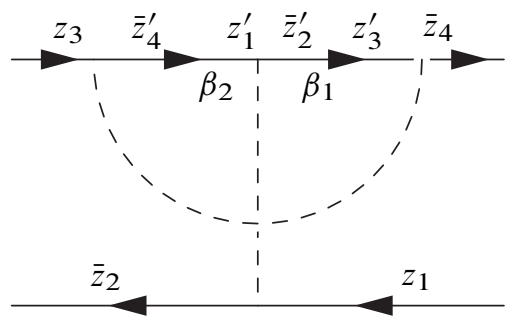

$$
\begin{aligned}
\Gamma_{4 k}^{(1)}\left[z_{1}, \bar{z}_{2}, z_{3}, \bar{z}_{4}\right] & \\
=- & \frac{\lambda^{\prime 2}}{4} \int_{\varepsilon}^{\infty} d \beta_{1} d \beta_{2} e^{-\frac{\mu_{0}^{2}}{2}\left(\beta_{1}+\beta_{2}\right)} \\
& \cdot e^{\omega\left(\bar{z}_{2} z_{1}+\bar{z}_{4} e^{-\left(\beta_{1}+\beta_{2}\right) \omega_{\left.z_{3}\right)}}\right.}
\end{aligned}
$$

All analogues of the second non-planar graph (4.33) are finite:

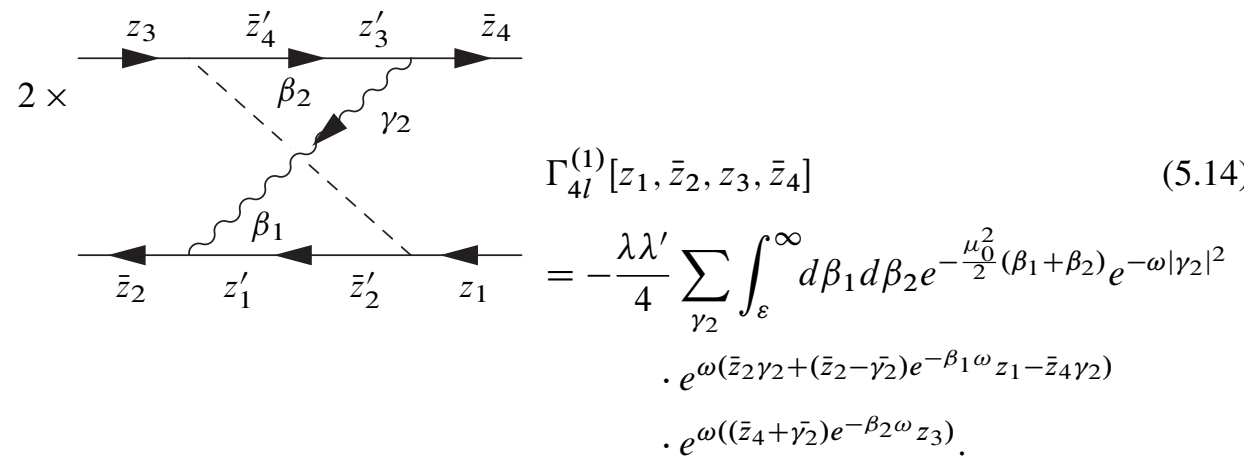




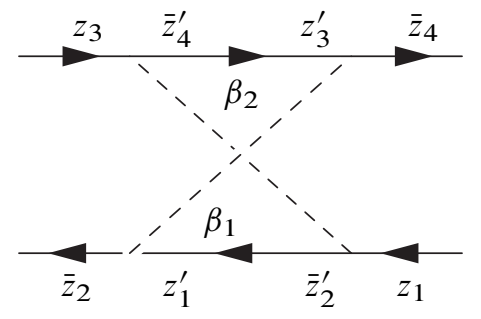

$$
\begin{aligned}
\Gamma_{4 m}^{(1)}\left[z_{1}, \bar{z}_{2}, z_{3}, \bar{z}_{4}\right] & \\
=- & \frac{\lambda^{\prime 2}}{8} \int_{\varepsilon}^{\infty} d \beta_{1} d \beta_{2} e^{-\frac{\mu_{0}^{2}}{2}\left(\beta_{1}+\beta_{2}\right)} \\
& \cdot e^{\omega\left(\bar{z}_{2} e^{-\beta_{1} \omega} z_{1}+\bar{z}_{4} e^{-\beta_{2} \omega} z_{3}\right)} .
\end{aligned}
$$

In conclusion the new divergences reproduce the action functional (4.38) (which includes the new vertex) so that the model is 1-loop renormalisable.

\section{6. $\beta$-functions}

Here we compute the $\beta$-functions of our model in the most interesting case where $\theta$ is irrational and satisfies the Diophantine condition (4.22). We can summarise the divergent Green's functions to

$$
\begin{gathered}
\Gamma_{2}^{(1)}\left[z_{1}, \bar{z}_{2}\right]=\frac{1}{2 \omega}\left(\lambda\left(1+\frac{1}{\theta^{2}}\right)+\lambda^{\prime}\right) I\left(z_{1}, \bar{z}_{2}\right)\left(\frac{1}{\epsilon \omega}+\left(1-\frac{\mu_{0}^{2}}{2 \omega}\right) \ln \frac{1}{\epsilon}\right) \\
\Gamma_{4}^{(1)}\left[z_{1}, \bar{z}_{2}, z_{3}, \bar{z}_{4}\right]=-\frac{\lambda}{4 \omega^{2}}\left(1+\frac{1}{\theta^{2}}\right) V\left(z_{1}, \bar{z}_{2}, z_{3}, \bar{z}_{4}\right) \ln \frac{1}{\epsilon} \\
-\frac{1}{8 \omega^{2}}\left(\lambda^{\prime 2}+\frac{3 \lambda^{2}}{\theta^{2}}+2 \lambda \lambda^{\prime}\left(1+\frac{1}{\theta^{2}}\right)\right) \\
\cdot I\left(z_{1}, \bar{z}_{2}\right) I\left(z_{3}, \bar{z}_{4}\right) \ln \frac{1}{\epsilon} .
\end{gathered}
$$

This leads to the following relation between the renormalised quantities $\mu_{R}, \lambda_{R}, \lambda_{R}^{\prime}$ and the bare quantities $\mu_{0}, \lambda, \lambda^{\prime}$ :

$$
\begin{aligned}
& \mu_{R}=\mu_{0}+\frac{1}{2 \omega}\left(\lambda\left(1+\frac{1}{\theta^{2}}\right)+\lambda^{\prime}\right)\left(\frac{1}{\epsilon \omega}+\left(1-\frac{\mu_{0}^{2}}{2 \omega}\right) \ln \frac{1}{\epsilon}\right), \\
& \lambda_{R}=\lambda-\frac{\lambda^{2}}{4 \omega^{2}}\left(1+\frac{1}{\theta^{2}}\right) \ln \frac{1}{\epsilon}, \\
& \lambda_{R}^{\prime}=\lambda^{\prime}-\frac{1}{4 \omega^{2}}\left(\lambda^{\prime 2}+\frac{3 \lambda^{2}}{\theta^{2}}+2 \lambda \lambda^{\prime}\left(1+\frac{1}{\theta^{2}}\right)\right) \ln \frac{1}{\epsilon} .
\end{aligned}
$$


Solving for the bare quantities, we obtain

$$
\begin{aligned}
\mu_{0} & =\mu_{R}-\frac{1}{2 \omega}\left(\lambda_{R}\left(1+\frac{1}{\theta^{2}}\right)+\lambda_{R}^{\prime}\right)\left(\frac{1}{\epsilon \omega}+\left(1-\frac{\mu_{0}^{2}}{2 \omega}\right) \ln \frac{1}{\epsilon}\right), \\
\lambda & =\lambda_{R}+\frac{\lambda_{R}^{2}}{4 \omega^{2}}\left(1+\frac{1}{\theta^{2}}\right) \ln \frac{1}{\epsilon}, \\
\lambda^{\prime} & =\lambda_{R}^{\prime}+\frac{1}{4 \omega^{2}}\left(\lambda_{R}^{\prime 2}+\frac{3 \lambda_{R}^{2}}{\theta^{2}}+2 \lambda_{R} \lambda_{R}^{\prime}\left(1+\frac{1}{\theta^{2}}\right)\right) \ln \frac{1}{\epsilon} .
\end{aligned}
$$

The $\beta$-functions are therefore given by

$$
\beta=\frac{\lambda_{R}^{2}}{4 \omega^{2}}\left(1+\frac{1}{\theta^{2}}\right), \quad \beta^{\prime}=\frac{1}{4 \omega^{2}}\left(\lambda_{R}^{\prime 2}+\frac{3 \lambda_{R}^{2}}{\theta^{2}}+2 \lambda_{R} \lambda_{R}^{\prime}\left(1+\frac{1}{\theta^{2}}\right)\right) .
$$

We thus conclude that the model is neither asymptotically free nor has a finite fixed point as it was the case for the renormalisable $\phi_{4}^{4}$-model on the Moyal plane [19]. The result also shows that there does not exist a ( $\theta$-independent) relation between the coupling constants $\lambda, \lambda^{\prime}$ which is preserved over all scales.

\section{Conclusion}

In the general formalism of noncommutative geometry, we have proposed a definition of a field theory on a projective module which is the noncommutative analogue of a scalar field theory with non-trivial topology. In the case of a noncommutative torus, the simplest non-trivial projective modules can be constructed using representations of the Heisenberg commutation relations. We have given a detailed account of the corresponding field theory, whose properties are reminiscent of those of a rectangular matrix model, whereas algebra-valued fields correspond to square matrices.

In particular, we have shown that the model suffers from an UV/IR-mixing. Contrary to what happens for field theories on the Moyal plane, here the arithmetical nature of the deformation parameters plays a central rôle. For $\theta$ satisfying a Diophantine condition, the theory is 1-loop renormalisable at the price of introducing the extra counterterm

$$
\left[\operatorname{Tr}_{\mathcal{A}_{\theta}}(\phi, \phi)_{\mathcal{A}_{\theta}}\right]^{2}
$$

in the action, whereas the basic action we started with is $\operatorname{Tr}_{\mathcal{A}_{\theta}}\left[(\phi, \phi)_{\mathcal{A}_{\theta}}^{2}\right]$. In a commutative field theory such a counterterm would break the locality of the action, but in a matrix model it corresponds to $\left[\operatorname{Tr}\left(M^{\dagger} M\right)\right]^{2}$ and is perfectly acceptable. This is the only way we found to cure the UV/IR-mixing problem. Note that the adjunction of such a counterterm fits perfectly with the spirit of [18]. The only difference is that they need to modify the propagator, whereas we need to change the interaction.

The occurrence of the Diophantine condition is not so surprising here. In fact, after suitable Poisson re-summations, divergences in the $\beta$-integrals appear whenever the 
lattice $\Gamma$ and its dual $\widehat{\Gamma}$ have elements that are close enough. In the rational case, the two lattices have non-trivial intersections that lead to sharp divergences corresponding to the usual counterterms, but otherwise the distance is bounded by a fixed number. In the irrational case, $\Gamma \cap \widehat{\Gamma}=\{0\}$, but the two lattices have elements that are as close as possible. The Diophantine condition allows to set a lower bound for the distance between $\Gamma$ and $\widehat{\Gamma}$ when restricted to a ball of radius $R$ as a function of $R$.

Acknowledgements. R. W. thanks the Université de Provence. J.-H. J. gratefully acknowledges his fellowship of the Friedrich-Ebert-Stiftung and wants to thank Prof. G. Grensing for helpful discussions.

\section{References}

[1] C. Becchi, S. Giusto, and C. Imbimbo, The Wilson-Polchinski renormalization group equation in the planar limit. Nuclear Phys. B 633 (2002), 250-270. Zbl 0995.81076 MR 1909062

[2] J. Bellissard, $C^{*}$ algebras in solid state physics: 2D electrons in a uniform magnetic field. In Operator algebras and applications, Vol. 2, London Math. Soc. Lecture Note Ser. 136, Cambridge University Press, Cambridge 1988, 49-76. Zbl 0677.46055 MR 0996451

[3] J. Bellissard, The noncommutative geometry of aperiodic solids. In Geometric and topological methods for quantum field theory (Villa de Leyva, 2001), World Scientific Publishing, Singapore 2003, 86-156. Zbl 1055.81034 MR 2009996

[4] A. H. Chamseddine and A. Connes, Scale invariance in the spectral action. J. Math. Phys. 47 (2006), 063504. Zbl 1112.83036 MR 2239979

[5] A. H. Chamseddine, A. Connes, and M. Marcolli, Gravity and the standard model with neutrino mixing. Preprint 2006, arXiv:hep-th/0610241

[6] I. Chepelev and R. Roiban, Convergence theorem for non-commutative Feynman graphs and renormalization. J. High Energy Phys. 03 (2001), 001. MR 1824728

[7] G. M. Cicuta, L. Molinari, E. Montaldi, and F. Riva, Large rectangular random matrices. J. Math. Phys. 28 (1987), 1716-1718. Zbl 0624.60120 MR 899170

[8] A. Connes, C* algèbres et géométrie différentielle. C. R. Acad. Sci. Paris Sér. A 290 (1980), 599-604; translated as ' $C^{*}$ algebras and differential geometry', arXiv:hep-th/0101093. Zbl 0433.46057 MR 0572645

[9] A. Connes, Non-commutative differential geometry. Inst. Hautes Études Sci. Publ. Math. 62 (1985), 257-360. Zbl 0592.46056 MR 823176

[10] A. Connes, Noncommutative geometry. Academic Press, San Diego, CA, 1994. Zbl 0818.46076 MR 1303779

[11] A. Connes, M. R. Douglas, and A. S. Schwarz, Noncommutative geometry and matrix theory: compactification on tori. J. High Energy Phys. 02 (1998), 003. Zbl 1018.81052 MR 1613978 
[12] A. Connes and M. Marcolli, A walk in the noncommutative garden. Preprint 2006, arXiv:math/0601054

[13] A. Connes and M. A. Rieffel, Yang-Mills for non-commutative two-tori. In Operator algebras and mathematical physics (Iowa City, Iowa, 1985), Contemp. Math. 62, Amer. Math. Soc., Providence, RI, 1987, 237-266. Zbl 0633.46069 MR 0878383

[14] L. Dąbrowski, G. Landi, A. Sitarz, W. van Suijlekom, and J. C. Várilly, The Dirac operator on $S U_{q}(2)$. Commun. Math. Phys. 259 (2005), 729-759. Zbl 1090.58504 MR 2174423

[15] T. Filk, Divergencies in a field theory on quantum space. Phys. Lett. B 376 (1996), 53-58. MR 1395558

[16] V. Gayral, Heat-kernel approach to UV/IR mixing on isospectral deformation manifolds. Ann. Henri Poincaré 6 (2005), 991-1023. Zbl 1082.58006 MR 2189376

[17] V. Gayral, B. Iochum, and D. V. Vassilevich, Heat kernel and number theory on NC-torus. Commun. Math. Phys. 273 (2007), 415-443. MR 2318313

[18] H. Grosse and R. Wulkenhaar, Renormalisation of $\phi^{4}$-theory on noncommutative $\mathbb{R}^{4}$ in the matrix base. Commun. Math. Phys. 256 (2005), 305-374. Zbl 1075.82005 MR 2160797

[19] H. Grosse and R. Wulkenhaar, Renormalisation of $\phi^{4}$-theory on non-commutative $\mathbb{R}^{4}$ to all orders. Lett. Math. Phys. 71 (2005), 13-26. Zbl 02228415 MR 2136734

[20] R. Gurau, J. Magnen, V. Rivasseau, and F. Vignes-Tourneret, Renormalization of noncommutative $\Phi_{4}^{4}$ field theory in $x$ space. Commun. Math. Phys. 267 (2006), 515-542. Zbl 1113.81101 MR 2249779

[21] R. Gurau and V. Rivasseau, Parametric representation of noncommutative field theory. Commun. Math. Phys. 272 (2007), 811-835. MR 2304476

[22] A. Kirillov, Geometric quantization. In Dynamical systems IV, 2nd ed., Encyclopaedia Math. Sci. 4, Springer-Verlag, Berlin 2001, 139-176. Zbl 0780.58024 MR 1866632

[23] T. Krajewski and R. Wulkenhaar, Perturbative quantum gauge fields on the noncommutative torus. Internat. J. Modern Phys. A 15 (2000), 1011-1029. Zbl 0963.81055 MR 1758227

[24] E. Langmann and R. J. Szabo, Duality in scalar field theory on noncommutative phase spaces. Phys. Lett. B 533 (2002), 168-177. Zbl 0994.81116 MR 1911944

[25] M. A. Rieffel, Projective modules over higher-dimensional noncommutative tori. Canad. J. Math. 40 (1988), 257-338. Zbl 0663.46073 MR 941652

[26] V. Rivasseau, F. Vignes-Tourneret, and R. Wulkenhaar, Renormalisation of noncommutative $\phi^{4}$-theory by multi-scale analysis. Commun. Math. Phys. 262 (2006), 565-594. Zbl 1109.81056 MR 2202303

[27] N. Seiberg and E. Witten, String theory and noncommutative geometry. J. High Energy Phys. 09 (1999), 032. Zbl 0957.81085 MR 1720697

[28] E. Witten, Non-commutative geometry and string field theory. Nuclear Phys. B 268(1986), 253-294. MR 834515

[29] E. Witten, Overview of $K$-theory applied to strings. Internat. J. Modern Phys. A 16 (2001), 693-706. Zbl 0980.81049 MR 1827946 
Received December 11, 2006

Victor Gayral, Matematisk Afdeling, Københavns Universitet, Universitetsparken 5, 2100 København, Denmark

E-mail: gayral@math.ku.dk

Jan-Hendrik Jureit, Centre de Physique Théorique, Unité Mixte de Recherche du CNRS (UMR 6207) et des Universités Aix-Marseille I, Aix-Marseille II et de l'Université du Sud Toulon-Var, Laboratoire affilié à la F.R.U.M.A.M. - FR 2291, France, and ChristianAlbrechts-Universität Kiel, Germany

E-mail: jureit@cpt.univ-mrs.fr

Thomas Krajewski, Centre de Physique Théorique, Unité Mixte de Recherche du CNRS (UMR 6207) et des Universités Aix-Marseille I, Aix-Marseille II et de l'Université du Sud Toulon-Var, Laboratoire affilié à la F.R.U.M.A.M. - FR 2291 and Université de Provence, France

E-mail: krajew@cpt.univ-mrs.fr

Raimar Wulkenhaar, Mathematisches Institut, Westfälische Wilhelms-Universität Münster, Einsteinstraße 62, 48149 Münster, Germany

E-mail: raimar@math.uni-muenster.de 